\title{
Status and Prospects of Top-Quark Physics
}

\author{
Joseph R. Incandela ${ }^{4}$, Arnulf Quadt ${ }^{1}$, Wolfgang Wagner ${ }^{2,5}$, \\ Daniel Wicke W,5 $^{3,}$ \\ ${ }^{1}$ Universität Göttingen, II. Physikalisches Institut, \\ 37077 Göttingen, Germany \\ ${ }^{2}$ Universität Karlsruhe, Institut für Experimentelle Kernphysik, \\ 76128 Karlsruhe, Germany \\ 3 Johannes Gutenberg Universität, Institut für Physik, \\ 55099 Mainz, Germany \\ ${ }^{4}$ University of California at Santa Barbara, Santa Barbara, \\ California 93106, Unites States of America \\ ${ }^{5}$ Bergische Universität Wuppertal, Fachgruppe Physik, \\ 42119 Wuppertal, Germany
}

November 5, 2018

\begin{abstract}
The top quark is the heaviest elementary particle observed to date. Its large mass of about $173 \mathrm{GeV} / c^{2}$ makes the top quark act differently than other elementary fermions, as it decays before it hadronises, passing its spin information on to its decay products. In addition, the top quark plays an important role in higher-order loop corrections to standard model processes, which makes the top quark mass a crucial parameter for precision tests of the electroweak theory. The top quark is also a powerful probe for new phenomena beyond the standard model.

During the time of discovery at the Tevatron in 1995 only a few properties of the top quark could be measured. In recent years, since the start of Tevatron Run II, the field of top-quark physics has changed and entered a precision era. This report summarises the latest measurements and studies of top-quark properties and gives prospects for future measurements at the Large Hadron Collider (LHC).
\end{abstract}

\section{Introduction}

There are six known quarks in nature, the up, down, strange, charm, bottom, and the top quark. The quarks are arranged in three pairs or "generations". Each member of a pair may be transformed into its partner via the charged-current weak interaction. Together with the six known leptons (the electron, muon, tau, and their associated neutrinos), the six quarks constitute all of the known luminous matter in the universe. The understanding of the properties of the quarks and leptons and their interactions is therefore of paramount importance. 
The top quark is the charge, $Q=+2 / 3$, and $T_{3}=+1 / 2$ member of the weak-isospin doublet containing the bottom quark. It is the most recently discovered quark, which was directly observed in 1995 by the CDF and DØ experiments at the FERmilab TEvatron, a $p \bar{p}$ collider at a centre-of-mass energy of $\sqrt{s}=1.8 \mathrm{TeV}$. This discovery was a great success of the Standard Model of Elementary Particle Physics (SM), which suggested the existence of the top quark as the weak-isospin partner of the $b$-quark already in 1977 at its discovery. Indirect evidence for the existence of the top quark became compelling over the years and constraints on the top quark mass, inferred from electroweak precision data, pointed exactly at the range where the top quark was discovered. Due to its relatively recent discovery, far less is known about the top quark than about the other quarks and leptons.

The strong and weak interactions of the top quark are not nearly as well studied as those of the other quarks and leptons. The strong interaction is most directly measured in top quark pair production. The weak interaction is measured in top quark decay and single top quark production. There are only a few fundamental parameters associated with the top quark in the SM: the top quark mass and the three CKM matrix elements involving top.

Thus far, the properties of the quarks and leptons are successfully described by the SM. However, this theory does not account for the masses of these particles, it merely accommodates them. Due to the mass of the top quark being by far the heaviest of all quarks, it is often speculated that it might be special amongst all quarks and leptons and might play a role in the mechanism of electroweak symmetry breaking. Even if the top quark turned out to be a SM quark, the experimental consequences of this very large mass are interesting in their own. Many of the measurements described in this review have no analogue for the lighter quarks. In contrast to the lighter quarks, which are permanently confined in bound states (hadrons) with other quarks and antiquarks, the top quark decays so quickly that it does not have time to form such bound states. There is also insufficient time to depolarise the spin of the top quark, in contrast to the lighter quarks, whose spin is depolarised by chromomagnetic interactions within the bound states. Thus the top quark is free of many of the complications associated with the strong interaction. Also, top quarks are and will remain a major source of background for almost all searches for physics beyond the Standard Model. Precise understanding of the top signal is crucial to claim new physics.

This review summarises the present knowledge of the properties of the top quark such as its mass and electric charge, its production mechanisms and rate and its decay branching ratios, etc., and provides a discussion of the experimental and theoretical issues involved in their determination. Earlier reviews on top quark physics at Run I or the earlier Run II can be found in [1, 2, 3, 4, 5, 6, 17, 8, 9, 10, 11].

Since the TEVATRON at FERMilab is today still the only place where top quarks can be produced and studied directly, most of the discussion in this article describes top quark physics at the TEVATRON. In particular, the focus is placed on the already available wealth of results from the Run II, which started in 2001 after a five year upgrade of the TEvaTRON collider and the experiments CDF and DØ. However, the Large Hadron Collider, LHC, a proton-proton collider at a centre-of-mass energy of $\sqrt{s}=10-14 \mathrm{TeV}$, commissioned with single-beam in September 2008 and planned to start collider operation at CERN in the fall 2009, will be a prolific source of top quarks and produce about 8 million $t \bar{t}$ events per year (at "low" luminosity, $10^{33} \mathrm{~cm}^{-2} \mathrm{~s}^{-1}$ ), a real "top factory". Measurements such as the top quark mass are entirely an issue of systematics, as the statistical uncertainty will be negligible.

\section{The Top Quark in the Standard Model}

\subsection{Brief Summary of the Standard Model}

Quantum field theory combines two great achievements of physics in the $20^{\text {th }}$-century, quantum mechanics and relativity. The SM [12, 13, 14, 15, 16, 17, 18, 19, 20, 21, 22, 23] is a particular quantum field 
theory, based on the set of fields shown in Table1, and the gauge symmetries $S U(3)_{C} \times S U(2)_{L} \times U(1)_{Y}$. There are three generations of quarks and leptons, labelled by the index $i=1,2,3$, and one Higgs field, $\phi$.

Table 1: The fields of the SM and their gauge quantum numbers. $T$ and $T_{3}$ are the total weak-isospin and its third component, and $Q$ is the electric charge.

\begin{tabular}{|c|c|c|c|c|c|c|c|c|c|}
\hline & & & & $S U(3)_{C}$ & $S U(2)_{L}$ & $U(1)_{Y}$ & $T$ & $T_{3}$ & $Q$ \\
\hline$Q_{L}^{i}=$ & $\left(\begin{array}{l}u_{L} \\
d_{L}\end{array}\right)$ & $\left(\begin{array}{l}c_{L} \\
s_{L}\end{array}\right)$ & $\left(\begin{array}{l}t_{L} \\
b_{L}\end{array}\right)$ & 3 & 2 & $1 / 6$ & $1 / 2$ & $\begin{array}{l}+1 / 2 \\
-1 / 2\end{array}$ & $\begin{array}{l}+2 / 3 \\
-1 / 3\end{array}$ \\
\hline$u_{R}^{i}=$ & $u_{R}$ & $c_{R}$ & $t_{R}$ & 3 & 1 & $2 / 3$ & 0 & 0 & $+2 / 3$ \\
\hline$d_{R}^{i}=$ & $d_{R}$ & $s_{R}$ & $b_{R}$ & 3 & 1 & $-1 / 3$ & 0 & 0 & $-1 / 3$ \\
\hline$L_{L}^{i}=$ & $\left(\begin{array}{c}\nu_{e L} \\
e_{L}\end{array}\right)$ & $\left.\begin{array}{c}\nu_{\mu_{L}} \\
\mu_{L}\end{array}\right)$ & $\left(\begin{array}{c}\nu_{\tau L} \\
\tau_{L}\end{array}\right)$ & 1 & 2 & $-1 / 2$ & $1 / 2$ & $\begin{array}{l}+1 / 2 \\
-1 / 2\end{array}$ & $\begin{array}{r}0 \\
-1\end{array}$ \\
\hline$e_{R}^{i}=$ & $e_{R}$ & $\mu_{R}$ & $\tau_{R}$ & 1 & 1 & -1 & 0 & 0 & -1 \\
\hline$\nu_{R}^{i}=$ & $\nu_{R}^{e}$ & $\nu_{R}^{\mu}$ & $\nu_{R}^{\tau}$ & 0 & 0 & 0 & 0 & 0 & 0 \\
\hline$\phi=$ & $\left(\begin{array}{l}\phi^{+} \\
\phi^{0}\end{array}\right)$ & & & 1 & 2 & $1 / 2$ & $1 / 2$ & $\begin{array}{l}+1 / 2 \\
-1 / 2\end{array}$ & $\begin{array}{r}+1 \\
0\end{array}$ \\
\hline
\end{tabular}

Once the gauge symmetries and the fields with their (gauge) quantum numbers are specified, the Lagrangian of the SM is fixed by requiring it to be gauge-invariant, local, and renormalisable. The SM Lagrangian can be divided into several pieces:

$$
\mathcal{L}_{S M}=\mathcal{L}_{\text {Gauge }}+\mathcal{L}_{\text {Matter }}+\mathcal{L}_{\text {Yukawa }}+\mathcal{L}_{\text {Higgs }}
$$

The first piece is the pure gauge Lagrangian, given by

$$
\mathcal{L}_{\text {Gauge }}=\frac{1}{2 g_{S}^{2}} \operatorname{Tr} G^{\mu \nu} G_{\mu \nu}+\frac{1}{2 g^{2}} \operatorname{Tr} W^{\mu \nu} W_{\mu \nu}-\frac{1}{4 g^{\prime 2}} B^{\mu \nu} B_{\mu \nu}
$$

where $G^{\mu \nu}, W^{\mu \nu}$, and $B^{\mu \nu}$ are the gluon, weak, and hypercharge field-strength tensors. These terms contain the kinetic energy of the gauge fields and their self interactions. The next piece is the matter Lagrangian, given by

$$
\mathcal{L}_{\text {Matter }}=i \bar{Q}_{L}^{i} \not D Q_{L}^{i}+i \bar{u}_{R}^{i} \not D u_{R}^{i}+i \bar{d}_{R}^{i} \not D d_{R}^{i}+i \bar{L}_{L}^{i} \not D L_{L}^{i}+i \bar{e}_{R}^{i} \not D e_{R}^{i} .
$$

This piece contains the kinetic energy of the fermions and their interactions with the gauge fields, which are contained in the covariant derivatives. For example,

$$
\not D Q_{L}=\gamma^{\mu}\left(\partial_{\mu}+i g_{S} G_{\mu}+i g W_{\mu}+i \frac{1}{6} g^{\prime} B_{\mu}\right) Q_{L}
$$

since the field $Q_{L}$ participates in all three gauge interactions. A sum on the index $i$, which represents the generations, is implied in the Lagrangian.

These two pieces of the Lagrangian depend only on the gauge couplings $g_{S}, g, g^{\prime}$. Their approximate values, evaluated as $M_{Z}$, are

$$
g_{S} \approx 1, \quad g \approx 2 / 3, \quad g^{\prime} \approx 2 /(3 \sqrt{3}) .
$$

Mass terms for the gauge bosons and the fermions are forbidden by the gauge symmetries. 
The next piece of the Lagrangian is the Yukawa interaction of the Higgs field with the fermions, given by

$$
\mathcal{L}_{\text {Yukawa }}=-\Gamma_{u}^{i j} \bar{Q}_{L}^{i} \epsilon \phi^{*} u_{R}^{j}-\Gamma_{d}^{i j} \bar{Q}_{L}^{i} \phi d_{R}^{j}-\Gamma_{e}^{i j} \bar{L}_{L}^{i} \phi e_{R}^{j}+h . c .
$$

where $\epsilon=i \sigma_{2}$ is the total antisymmetric tensor in 2 dimensions, related to the second Pauli matrix $\sigma_{2}$ and required to ensure each term separately to be electrically neutral, and the coefficients $\Gamma_{u}, \Gamma_{d}, \Gamma_{e}$ are $3 \times 3$ complex matrices in generation space. They need not be diagonal, so in general there is mixing between different generations. These matrices contain most of the parameters of the SM.

The final piece is the Higgs Lagrangian [24, 25, 26], given by

$$
\mathcal{L}_{\text {Higgs }}=\left(D^{\mu} \phi\right)^{\dagger} D_{\mu} \phi+\mu^{2} \phi^{\dagger} \phi-\lambda\left(\phi^{\dagger} \phi\right)^{2}
$$

with the Higgs doublet $\phi$ as given in Table 1. This piece contains the kinetic energy of the Higgs field, its gauge interactions, and the Higgs potential. The coefficient of the quadratic term, $\mu^{2}$, is the only dimensionful parameter in the SM. The sign of this term is chosen such that the Higgs field has a non-zero vacuum-expectation value on the circle of minima in Higgs-field space given by $\left\langle\phi^{0}\right\rangle=\mu / \sqrt{2 \lambda} \equiv v / \sqrt{2}$. The dimensionful parameter $\mu$ is replaced by the dimensionful parameter $v \approx 246 \mathrm{GeV}$.

The acquisition of a non-zero vacuum-expectation value by the Higgs field breaks the electroweak symmetry and generates masses for the gauge bosons,

$$
M_{W}=\frac{1}{2} g v, \quad M_{Z}=\frac{1}{2} \sqrt{g^{2}+g^{\prime 2}} v, \quad \text { and for the fermions, } M_{f}=\Gamma_{t} \frac{v}{\sqrt{2}},
$$

with the Yukawa coupling $\Gamma_{t}$. Diagonalising the fermion mass matrices generates the Cabibbo-Kobayashi-Maskawa (CKM) matrix [27, 28], including the CP-violating phase.
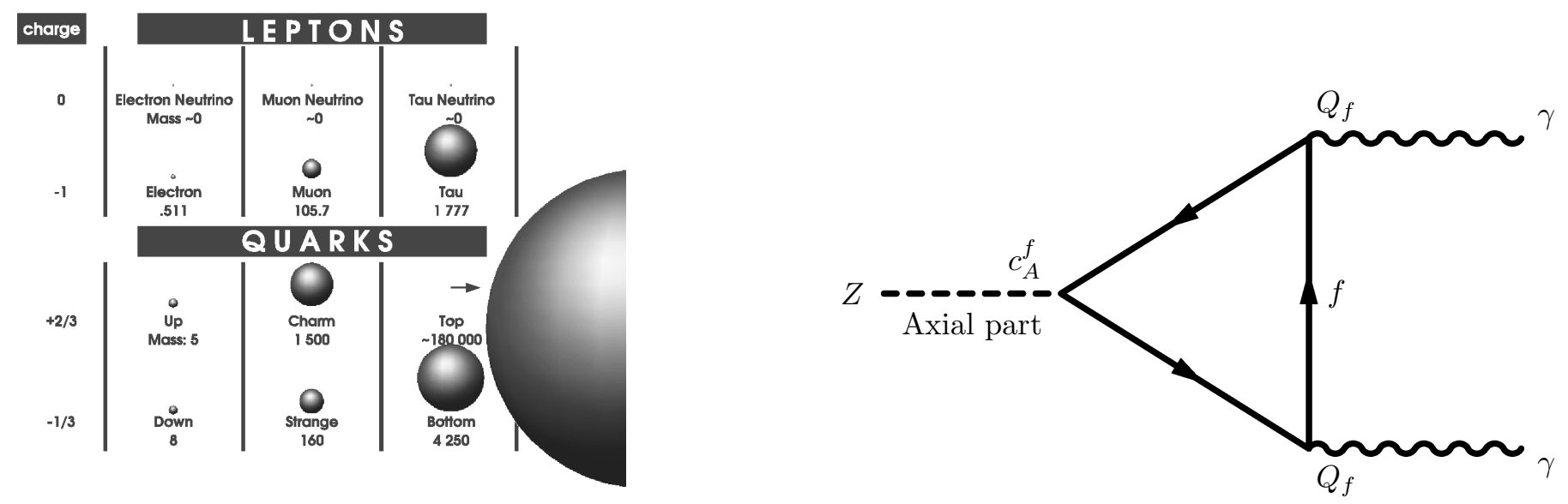

Figure 1: Left: Table of lepton and quark properties such as electric charge and mass (in $\mathrm{MeV} / \mathrm{c}^{2}$ ). The top quark is unique amongst all fermions due to its very large mass. (All elementary particles are point-like. The relative size of the drawn spheres merely symbolizes the mass of fermions, but does not scale linearly with the fermion mass.) Right: A fermion (quark or lepton) triangle diagram which potentially could cause an anomaly.

Figure 1 shows three lepton and quark families with their electric charge and approximate mass. While the neutrinos have non-zero, but very small masses, the quark masses are much larger. The top quark, with a mass of $\approx 175 \mathrm{GeV} / \mathrm{c}^{2}$, is by far the heaviest fermion. 


\subsection{Indirect Evidence for the Existence of the Top Quark}

In 1977, the $b$-quark was discovered at Fermilab [29]. The existence of a weak isospin partner of the $b$-quark, the top quark, was anticipated for two main reasons: First it provides a natural way to suppress the experimentally not observed flavour-changing neutral current. The argument on which the GIM mechanism [15] is based applies just as well for three as for two quark doublets.

The second reason is concerned with the desire to obtain a renormalisable gauge theory of weak interactions. The SM of electroweak interactions can be proven to be renormalisable under the condition that the sum of the weak hypercharges, $Y_{i}$, of all left-handed fermions is zero, i.e.

$$
\sum_{\substack{\text { left-handed } \\ \text { arks and leptons }}} Y_{i}=0 .
$$

Since every lepton multiplet contributes a value of $y=-2$ and every quark multiplet a value of $+2 / 3$, the sum only vanishes if there are three colours, i.e. every quark exists in three colour versions, and if the number of quark flavours equals the number of lepton species. The general proof that gauge theories can be renormalised, however, can only be applied if the particular gauge theory is anomaly fres. This requires a delicate cancellation between different diagrams, relations which can easily be upset by "anomalies" due to fermion loops such as the one shown in Figure 1. The major aspect is an odd number of axial-vector couplings. In general, anomaly freedom is guaranteed if the coefficient 3

$$
d_{a b c}=\sum_{\text {fermions }} \operatorname{Tr}\left[\hat{\lambda}^{a}\left\{\hat{\lambda}^{b}, \hat{\lambda}^{c}\right\}_{+}\right]=0
$$

where the $\hat{\lambda}^{i}$ are in general the generators of the gauge group under consideration. In the SM of electroweak interactions, the gauge group $S U(2) \times U(1)$ is generated by the three Pauli matrices, $\sigma_{i}$, and the hypercharge $Y: \hat{\lambda}^{i}=\sigma_{i}$, for $i=1,2,3$, and $\hat{\lambda}^{4}=\hat{Y}=2\left(\hat{Q}-\hat{T}_{3}\right)$. In the specific example shown in Figure [1, one consequence of Equation [10 is a relation where each triangle is proportional to $c_{A}^{f} Q_{f}^{2}$, where $Q_{f}$ is the charge and $c_{A}^{f}$ is the axial coupling of the weak neutral current. Thus, for an equal number $N$ of lepton and quark doublets, the total anomaly is proportional to:

$$
d \propto \sum_{i=1}^{N}\left(\frac{1}{2} \cdot(0)^{2}-\frac{1}{2} \cdot(-1)^{2}+\frac{1}{2} \cdot N_{c} \cdot\left(+\frac{2}{3}\right)^{2}-\frac{1}{2} \cdot N_{c} \cdot\left(-\frac{1}{3}\right)^{2}\right) .
$$

Consequently, taking into account the three colours of each quark $\left(N_{c}=3\right)$, the anomalies are cancelled. Since three lepton doublets were observed many years ago (the tau neutrino was experimentally only observed directly in the year 2000, but the number of light neutrino generations was known to be 3 from the LEP data on the $Z$-pole), the lack of anomalies such as the one shown in Figure 1 therefore requires the existence of the three quark doublets.

There is a lot of indirect experimental evidence for the existence of the top quark. The experimental limits on flavour changing neutral current (FCNC) decays of the $b$-quark [30, 31] such as $b \rightarrow s \ell^{+} \ell^{-}$ and the absence of large tree level (lowest order) $B_{d}^{0} \bar{B}_{d}^{0}$ mixing at the $\Upsilon(4 S)$ resonance [32, 33, 34, 35]

\footnotetext{
${ }^{1}$ The gauge theory has to be consistent, i.e. anomaly-free, in order to be at least unitary. The requirement of the gauge theory to be renormalisable is stronger than to be consistent, but the former argument is more familiar to most readers. The important consequence of both requirements is that the gauge theory is anomaly-free.

${ }^{2}$ A gauge theory might be renormalisable, whether or not it is anomaly free. The general proof of renormalisability, however, cannot be applied if it is not.

${ }^{3} d_{a b c}$ is the coefficient in the definition of the anomaly: $\left[\partial_{\mu} J_{\alpha}^{\mu}(x)\right]_{\text {anom }}=-\frac{1}{32 \pi^{2}} \cdot d_{\alpha \beta \gamma} \epsilon^{\kappa \nu \lambda \rho} \cdot F_{\kappa \nu}^{\beta}(x) \cdot F_{\lambda \rho}^{\gamma}(x)$, with the current $J_{\alpha}^{\mu}(x)$, the field strength tensor $F_{\kappa \nu}^{\beta}$ and the total antisymmetric tensor $\epsilon^{\kappa \nu \lambda \rho}$.
} 
rule out the hypothesis of an isosinglet $b$-quark. In other words, the $b$-quark must be a member of a left-handed weak isospin doublet.

The most compelling argument for the existence of the top quark comes from the wealth of data accumulated at the $e^{+} e^{-}$colliders LEP and SLC in recent years, particularly the detailed studies of the $Z b \bar{b}$ vertex near the $Z$ resonance [36]. These studies have yielded a measurement of the isospin of the $b$-quark. The $Z$-boson is coupled to the $b$-quarks (as well as the other quarks) through vector and axial vector charges $\left(v_{b}\right.$ and $\left.a_{b}\right)$ with strength (Feynman diagram vertex factor)

$$
\begin{aligned}
Z \sim \gamma_{b} & =\frac{-i g}{\cos \theta_{W}} \gamma^{\mu} \frac{1}{2}\left(v_{b}-a_{b} \gamma^{5}\right) \\
& =-i \sqrt{\sqrt{2} G_{F} M_{Z}^{2}} \gamma^{\mu}\left(v_{b}-a_{b} \gamma^{5}\right),
\end{aligned}
$$

where $v_{b}$ and $a_{b}$ are given by

$$
\begin{aligned}
v_{b} & =\left[T_{3}^{L}(b)+T_{3}^{R}(b)\right]-2 e_{b} \sin ^{2} \theta_{W}, \quad \text { and } \\
a_{b} & =\left[T_{3}^{L}(b)+T_{3}^{R}(b)\right] .
\end{aligned}
$$

Here, $T_{3}^{L}(b)$ and $T_{3}^{R}(b)$ are the third components of the weak isospin for the left-handed and righthanded $b$-quark fields, respectively. The electric charge of the $b$-quark, $e_{b}=-1 / 3$, has been well established from the $\Upsilon$ leptonic width as measured by the DoRIS $e^{+} e^{-}$experiment [37, 38, 39]. Therefore, measurements of the weak vector and axial-vector coupling of the $b$-quark, $v_{b}$ and $a_{b}$, can be interpreted as measurements of its weak isospin.

The (improved) Born approximation for the partial $Z$-boson decay rate gives in the limit of a zero mass b-quark:

$$
\Gamma_{b \bar{b}} \equiv \Gamma(Z \rightarrow b \bar{b})=\frac{G_{F} M_{Z}^{3}}{2 \sqrt{2} \pi}\left(v_{b}^{2}+a_{b}^{2}\right)
$$

The partial width $\Gamma_{b \bar{b}}$ is expected to be thirteen times smaller if $T_{3}^{L}(b)=0$. The LEP measurement of the ratio of this partial width to the full hadronic decay width, $R_{b}=\Gamma_{b} / \Gamma_{\text {had }}=0.21629 \pm 0.00066$ [36], is in excellent agreement with the SM expectation (including the effects of the top quark) of 0.2158 , ruling out $T_{3}^{L}(b)=0$. The sensitivity of $R_{b}$ to the mass of the top quark also shows that a top quark with a mass around $m_{t} \approx 175 \mathrm{GeV} / \mathrm{c}^{2}$ is strongly favoured [36].

In addition, the forward-backward asymmetry in $e^{+} e^{-} \rightarrow b \bar{b}$ below [40] and at the $Z$ pole [36],

$$
A_{F B}^{0}\left(M_{Z}\right)=\frac{3}{4} \frac{2 v_{e} a_{e}}{\left(v_{e}^{2}+a_{e}^{2}\right)} \frac{2 v_{b} a_{b}}{\left(v_{b}^{2}+a_{b}^{2}\right)}
$$

measured to be $A_{F B}^{0, b}=0.0992 \pm 0.0016$ is sensitive [36, 40] to the relative size of the vector and axial vector couplings of the $Z b \bar{b}$ vertex. The sign ambiguity for the two contributions can be resolved by the $A_{F B}$ measurements from low energy experiments that are sensitive to the interference between neutral current and electromagnetic amplitudes. From earlier measurements of $\Gamma_{b \bar{b}}$ and $A_{F B}$ at LEP, SLC, and the low energy experiments (PeP, Petra and Tristan [41, 42, 40]), one obtains [43]

$$
\begin{array}{ll}
T_{3}^{L}(b)=-0.490{ }_{-0.012}^{+0.015} & \Rightarrow T_{3}^{L}(b)=-1 / 2, \\
T_{3}^{R}(b)=-0.028 \pm 0.056 & \Rightarrow T_{3}^{R}(b)=0
\end{array}
$$

for the third component of the isospin of the $b$-quark. This implies that the $b$-quark must have a weak isospin partner, i.e. the top quark with $T_{3}^{L}(t)=+1 / 2$ must exist. 


\subsection{Indirect Constraints on the Mass of the Top Quark}

The precise electroweak measurements performed at LEP, SLC, NuTeV and the $p \bar{p}$ colliders can be used to check the validity of the SM and within its framework, to infer valuable information about its fundamental parameters. Due to the accuracy of those measurements, sensitivity to the mass of the top quark and the Higgs boson through radiative corrections is gained.

All electroweak quantities (mass, width and couplings of the $W$ - and the $Z$-boson) depend in the SM only on five parameters. At leading, order this dependence is reduced to only three parameters, two gauge couplings and the Higgs-field vacuum expectation value. The three best-measured electroweak quantities can be used to determine these three parameters: The electromagnetic coupling constant $\alpha$, measured in low-energy experiments [44], the Fermi constant, $G_{F}$ determined from the $\mu$ lifetime [45], and the mass of the $Z$-boson, measured in $e^{+} e^{-}$annihilation at LEP and SLC [36]. By defining the electroweak mixing angle $\theta_{W}$ through $\sin ^{2} \theta_{W} \equiv 1-m_{W}^{2} / m_{Z}^{2}$, the $W$-boson mass can be expressed as:

$$
m_{W}^{2}=\frac{\frac{\pi \alpha}{\sqrt{2} G_{F}}}{\sin ^{2} \theta_{W} \cdot(1-\Delta r)},
$$

where $\Delta r$ contains all the one-loop corrections. Contributions to $\Delta r$ originate from the top quark by the one-loop diagrams shown in Figure 2 (left), which contribute to the $W$ and $Z$ masses via:

$$
(\Delta r)_{t o p} \simeq-\frac{3 G_{F}}{8 \sqrt{2} \pi^{2} \tan ^{2} \theta_{W}} m_{t}^{2} .
$$
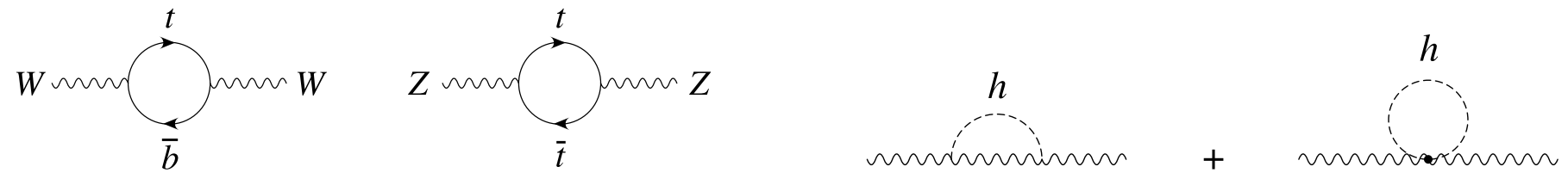

Figure 2: Left: Virtual top quark loops contributing to the $W$ and $Z$ boson masses. Right: Virtual Higgs boson loops contributing to the $W$ and $Z$ boson masses.

Also the Higgs boson contributes to $\Delta r$ via the one-loop diagrams, shown in Figure 2 (right):

$$
(\Delta r)_{H i g g s} \simeq \frac{3 G_{F} m_{W}^{2}}{8 \sqrt{2} \pi^{2}}\left(\ln \frac{m_{H}^{2}}{m_{Z}^{2}}-\frac{5}{6}\right) .
$$

While the leading $m_{t}$ dependence is quadratic, i.e. very strong, the leading $m_{H}$ dependence is only logarithmic, i.e. rather weak. Therefore the inferred constraints on $m_{H}$ are much weaker than those on $m_{t}$. This was used to successfully predict the top quark mass from the electroweak precision data before it was discovered by CDF and D $\varnothing$ in 1995 [46, 47]. Neutral current weak interaction data, such as $e^{+} e^{-}$annihilation near the $Z$ pole, $\nu N$ and $e N$ deep-inelastic scattering, $\nu e$ elastic scattering, and atomic parity violation can also be used to constrain the top quark mass.

The most recent indirect measurements of the top quark mass using the $Z$-pole data together with the direct measurements of the $W$-boson mass and total width and several other electroweak quantities yields [48, 49]:

$$
m_{\text {top }}=178.9_{-8.6}^{+11.7} \mathrm{GeV} / \mathrm{c}^{2}
$$

which is in very good agreement with the world average of the direct measurements [50]

$$
m_{\text {top }}=173.1 \pm 1.3 \mathrm{GeV} / \mathrm{c}^{2} .
$$


The global fit to all electroweak precision data including the world average of the direct top quark mass measurements yields [48, 49] the identical value as the direct measurement dominates the fit, while a fit only to the $Z$-pole data gives [36]:

$$
m_{\text {top }}=172.6_{-10.2}^{+13.2} \mathrm{GeV} / \mathrm{c}^{2}
$$

The successful prediction of the mass of the top quark before its discovery provides confidence in the precision and predictive power of radiative corrections in the SM. Therefore, the SM fit to the electroweak precision data including the direct measurements of the top quark and $W$-boson mass is used to infer on the mass of the SM Higgs boson. Figure 3 (left) shows the $\Delta \chi^{2}$ of the latest fit as a function of the Higgs boson mass. The most likely value of the Higgs mass, determined from the minimum of the $\Delta \chi^{2}$ curve is $90{ }_{-27}^{+36} \mathrm{GeV} / \mathrm{c}^{2}$ [48, 49], clearly indicating that the data prefers a light Higgs boson, $m_{H}<163 \mathrm{GeV} / \mathrm{c}^{2}$ at the $95 \%$ C.L. [48, 49]. The preferred value is slightly below the exclusion limit of $114.4 \mathrm{GeV} / \mathrm{c}^{2}$ at the $95 \%$ C.L. from the direct search for the SM Higgs boson at LEP [51] and at the TEVATRON [52].
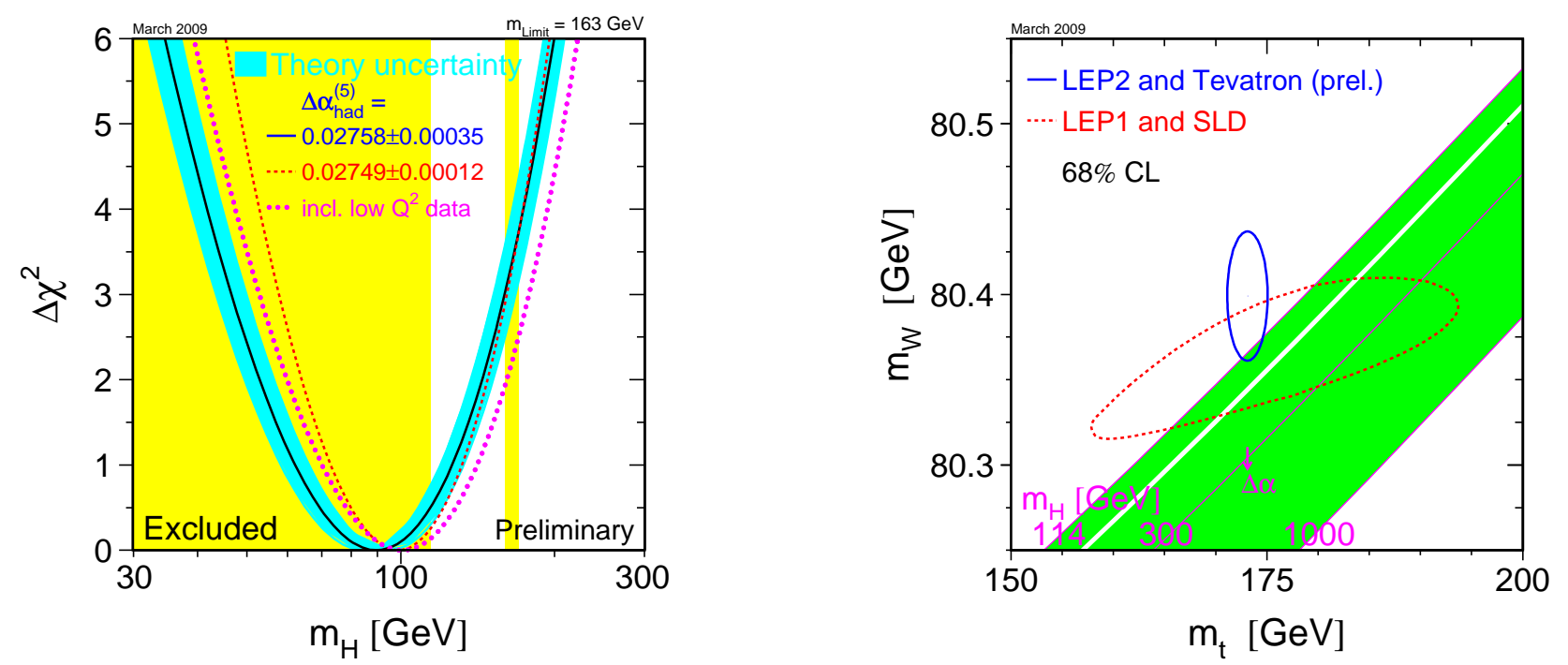

Figure 3: Left: Blueband plot, showing the indirect determination of the Higgs boson mass from all electroweak precision data together with the 95\% C.L. limit on the Higgs boson mass from the direct searches in yellow [51, 52]. Right: Lines of constant Higgs mass on a plot of $M_{W}$ vs. $m_{t}$. The dotted ellipse is the $68 \%$ C.L. direct measurement of $M_{W}$ and $m_{t}$. The solid ellipse is the $68 \%$ C.L. indirect measurement from precision electroweak data.

Figure 3 (right) shows the $68 \%$ C.L. contour in the $\left(m_{t}, m_{W}\right)$ plane from the global electroweak fit [48, 49]. It shows the direct and indirect determination of $m_{t}$ and $m_{W}$. Also displayed are the isolines of SM Higgs boson mass between the lower limit of $114 \mathrm{GeV} / \mathrm{c}^{2}$ and the theoretical upper limit of $1000 \mathrm{GeV} / \mathrm{c}^{2}$. As can be seen from the figure, the direct and indirect measurements are in good agreement, showing that the SM is not obviously wrong. On the other hand, the fit to all data has a $\chi^{2}$ per degree of freedom of $17.4 / 13$, corresponding to a probability of $18.1 \%$. This is mostly due to three anomalous measurements: the $b$ forward-backward asymmetry $\left(A_{F B}^{b}\right)$ measured at LEP, which deviates by $2.9 \sigma$, the total hadronic production cross section $\left(\sigma_{\text {had }}^{0}\right)$ at the $Z$-pole from LEP and the left-right cross section asymmetry $\left(A_{L R}\right)$ measured at SLC, both of which deviate from the SM fit value by about $1.5 \sigma$. If $\sin ^{2} \theta_{W}(\nu N)$, measured by the $\mathrm{NuTeV}$ collaboration [53], is in addition included in the fit, the measured and fitted value of $\sin ^{2} \theta_{W}(\nu N)$ differ by almost $3 \sigma$. It seems there is some tension in the fit of the precision electroweak data to the SM. 
Measurements of $M_{W}$ and $m_{t}$ at the TEVATRON could resolve or exacerbate this tension. Improvements in the precision of the measurement of the top quark or the $W$-boson mass at the TEVATRON translate into better indirect limits on the Higgs boson mass. This will also be a service to the LHC experiments which optimise their analysis techniques and strategies for the search for the yet elusive SM Higgs boson in the lower mass range, preferred by the SM electroweak fit.

Finally, in 1995, both CDF and DØ published the discovery of the top quark in strong $t \bar{t}$ production [46, 47], which marked the beginning of a new era, moving on from the search for the top quark to the studies and measurements of the properties of the top quark. Figure 4 shows the development of limits and measurements on the top quark mass from indirect and direct studies at $e^{+} e^{-}$and hadron colliders. The top quark was discovered with a mass of exactly the value that was predicted from global fits to electroweak precision data.

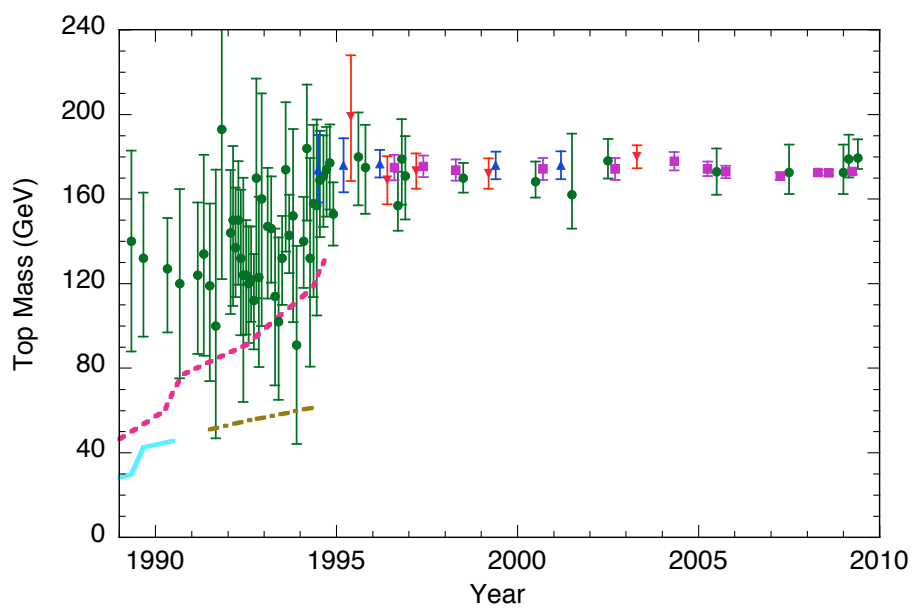

Figure 4: History of the limits on or measurements of the top quark mass (updated April 2009 by C. Quigg from [54]: (•) Indirect bounds on the top-quark mass from precision electroweak data; ( $)$ World-average direct measurement of the top-quark mass (including preliminary results); $(\mathbf{\Delta})$ published $\mathrm{CDF}$ and $(\boldsymbol{\nabla})$ D $\varnothing$ measurements; Lower bounds from $p \bar{p}$ colliders Sp$\overline{\mathrm{p}} \mathrm{S}$ and the TEvaTron are shown as dash-dotted and dashed lines, respectively, and lower bounds from $e^{+} e^{-}$colliders (PETRA, TRISTAN, LEP and SLC) are shown as a solid light grey line.

\subsection{Top-Quark Properties}

The mass of the top quark is larger than that of any other quark. Furthermore, the top quark mass is measured with better relative precision (0.75\%) than any other quark [50]. Given the experimental technique used to extract the top mass, these mass values should be taken as representing the top pole mass. The top pole mass, like any quark mass, is defined up to an intrinsic ambiguity of order $\Lambda_{Q C D} \sim$ $200 \mathrm{MeV}$ [55]. The most recent combination of top quark mass measurements by the TEVATRON Electroweak/Top Working group, including preliminary CDF and D $\varnothing$ measurements from Run II, yields $m_{t}=173.1 \pm 1.3 \mathrm{GeV} / \mathrm{c}^{2}\left[50\right.$. The prospects for the precision of $m_{t}$ measurements at the TEVATRON have recently been revised to better than $1.5 \mathrm{GeV} / \mathrm{c}^{2}$ per experiment with the full Run II data set. At the LHC, a precision of the top-quark mass measurement of $1-2 \mathrm{GeV} / \mathrm{c}^{2}$ is expected. At a future linear $e^{+} e^{-}$collider, the expected precision of a measurement of $m_{t}$ from a cross section scan at the $t \bar{t}$ production threshold is $\Delta m_{t}=20-100 \mathrm{MeV} / \mathrm{c}^{2}$ [56, 57, 58].

Like most of its fundamental quantum numbers, the electric charge of the top quark, $q_{t o p}$, has not been measured so far. The electric charge of the top quark is easily accessible in $e^{+} e^{-}$production by measurements of the ratio $R=\frac{e^{+} e^{-} \rightarrow \text { hadrons }}{e^{+} e^{-} \rightarrow \mu^{+} \mu^{-}}$through the top quark production threshold. However, 
this region of energy is not yet available at $e^{+} e^{-}$colliders. Thus, alternative interpretations for the particle that is believed to be the charge $2 / 3$ isospin partner of the $b$ quark are not ruled out. For example, since the correlations of the $b$ quarks and the $W$ bosons in $p \bar{p} \rightarrow t \bar{t} \rightarrow W^{+} W^{-} b \bar{b}$ events are not determined by CDF or D $\varnothing$, it is conceivable that the " $t$ quark" observed at the TEVATRON is an exotic quark, $Q_{4}$, with charge $-4 / 3$ with decays via $Q_{4} \rightarrow W^{-} b$ [59, 60, 61]. This interpretation is consistent with current precision electroweak data. In order to determine the charge of the top quark, one can either measure the charge of its decay products, in particular of the $b$ jet via jet charge techniques, or investigate photon radiation in $t \bar{t}$ events [62]. The latter method actually measures a combination of the electromagnetic coupling strength and the charge quantum number. Combining the results of the two methods will thus make it possible to determine both quantities.

At the Tevatron, with an integrated luminosity of $1-2 \mathrm{fb}^{-1}$, one is able to exclude at $95 \% \mathrm{CL}$ the possibility that an exotic quark $Q_{4}$ with charge $-4 / 3$ and not the SM top quark was found in Run I. At the LHC with $10 \mathrm{fb}^{-1}$ obtained at $10^{33} \mathrm{~cm}^{-2} \mathrm{~s}^{-1}$, it is expected to be possible to measure the electric charge of the top quark with an accuracy of $10 \%$. For comparison, at a linear collider with $\sqrt{s}=500 \mathrm{GeV}$ and $\int \mathcal{L} d t=200 \mathrm{fb}^{-1}$, one expects that $q_{t o p}$ can be measured with a precision of about $10 \%$ [63, 64].

The SM dictates that the top quark has the same vector-minus-axial-vector $(V-A)$ charged-current

weak interaction $\left(-i \frac{g}{\sqrt{2}} V_{t b} \gamma^{\mu} \frac{1}{2}\left(1-\gamma_{5}\right)\right)$ as all the other fermions. Neglecting the $b$-quark mass this implies that the $W$ boson in top-quark decay cannot be right-handed, i.e. have positive helicity.

One of the unique features of the top quark is that on average the top quark decays before there is time for its spin to be depolarised by the strong interaction [65]. Thus, the top quark polarisation 4 is directly observable via the angular distribution of its decay products. This means that it should be possible to measure observables that are sensitive to the top quark spin via spin correlations [66, 67, 68, 69, 70, 71, 72, 73, and references therein.

Another interesting aspect of the production of $t \bar{t}$ pairs via the strong coupling is an asymmetry in the rapidity-distribution of the $t$ and $\bar{t}$ quarks [74, 75, 76]. This effect arises at next-to-leading order, and leads to a forward-backward asymmetry of about $5 \%$ in $t \bar{t}$ production at the TEVATRON.

Yukawa coupling is the Higgs coupling to fermions and thus relates the fermionic matter content of the SM to the source of mass generation, the Higgs sector [24, 25, 26]. In the SM, the Yukawa coupling to the top quark, $y_{t}=\sqrt{2} m_{t} / v$, is very close to unity. This theoretically interesting value leads to numerous speculations that new physics might be accessed via top quark physics [77]. The Yukawa coupling will be measured in the associated $t \bar{t} H$ production at the LHC [78, 79, 80].

\section{Top-Quark Production and Decay}

In this chapter, we summarize the phenomenology of top-quark production at hadron colliders, limiting the discussion to SM processes. Anomalous top-quark production and non-SM decays are covered in chapter 9. A detailed, recent review on top-quark phenomenology can be found in [10]. The dominating source of top quarks at the Fermilab Tevatron and the Large Hadron Collider (LHC) at CERN is the production of $t \bar{t}$ pairs by the strong interaction, while the production of single top-quarks due to charged-current weak interactions has subleading character.

The Factorization Ansatz The underlying theoretical framework for the calculation of hadronic cross sections is the QCD-improved parton model which regards a high-energy hadron $A$ as a composition of quasi-free partons (quarks and gluons) sharing the hadron momentum $p_{A}$. Each parton $i$ carries a different momentum fraction $x_{i}$ and its momentum is given by $p_{i}=x_{i} p_{A}$. Based on the factorization

\footnotetext{
${ }^{4}$ The spin of an individual top quark cannot be measured, only the spin polarisation of an ensemble of top quarks.
} 


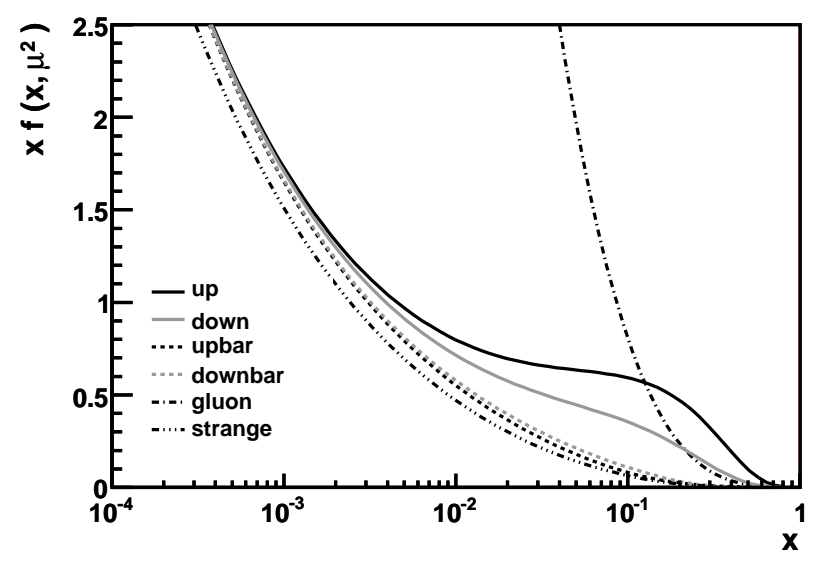

(a)

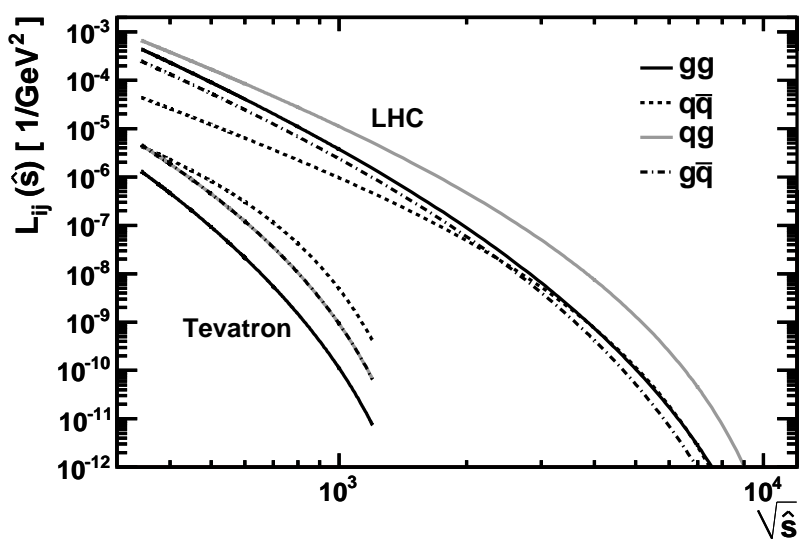

(b)

Figure 5: (a) PDFs of $u$ quarks, $\bar{u}$ quarks, $d$ quarks, $\bar{d}$ quarks, $s$ quarks, and gluons inside the proton. The parametrization is CTEQ6.5M [84]. The scale at which the PDFs are evaluated was chosen to be $\mu=175 \mathrm{GeV}\left(\mu^{2}=30625 \mathrm{GeV}^{2}\right)$. (b) Parton luminosities for gluon-gluon, quark-antiquark, quarkgluon, and gluon-antiquark interactions at the TEVATRON and the LHC [88].

theorem cross sections are calculated as a convolution of parton distribution functions (PDF) $f_{i / A}\left(x_{i}, \mu^{2}\right)$ and $f_{j / B}\left(x_{j}, \mu^{2}\right)$ for the colliding hadrons $(A, B)$ and the factorized hard parton-parton cross section $\hat{\sigma}_{i j}$ :

$$
\sigma(A B \rightarrow t \bar{t})=\sum_{i, j=q, \bar{q}, g} \int \mathrm{d} x_{i} \mathrm{~d} x_{j} f_{i / A}\left(x_{i}, \mu^{2}\right) f_{j / B}\left(x_{j}, \mu^{2}\right) \cdot \hat{\sigma}_{i j}\left(i j \rightarrow t \bar{t} ; \hat{s}, \mu^{2}\right)
$$

The hadrons $A B$ are either $p \bar{p}$ at the TEvatron or $p p$ at the LHC. The variable $\hat{s}$ denotes the square of the centre-of-mass energy of the colliding partons: $\hat{s}=\left(p_{i}+p_{j}\right)^{2}=\left(x_{i} p_{A}+x_{j} p_{B}\right)^{2}$. Neglecting terms proportional to the hadron masses we have $\hat{s}=x_{i} x_{j} s$. The sum in (25) runs over all pairs of partons $(i, j)$ contributing to the process. The PDF $f_{i / A}\left(x_{i}, \mu^{2}\right)$ describes the probability density for finding a parton $i$ inside the hadron $A$ carrying a momentum fraction $x_{i}$. The PDFs as well as $\hat{\sigma}_{i j}$ have a residual dependence on the factorization and renormalization scale $\mu$ due to uncalculated higher orders. In the case of top-quark production, one typically evaluates the cross sections at $\mu=m_{t}$. Strictly speaking one has to distinguish between the factorization scale $\mu_{\mathrm{F}}$ introduced by the factorization ansatz and the renormalization scale $\mu_{\mathrm{R}}$ due to the renormalization procedure invoked to regulate divergent terms in the perturbation series when calculating $\hat{\sigma}_{i j}$. However, since both scales are to some extent arbitrary parameters most authors have adopted the practice to use only one scale $\mu=\mu_{\mathrm{F}}=\mu_{\mathrm{R}}$. If the complete perturbation series could be calculated, the result for the cross section would be independent of $\mu$. However, at finite order in perturbation theory cross-section predictions do depend on the choice of $\mu$, and the changes when varying $\mu$ between $m_{t} / 2$ and $2 m_{t}$ are usually quoted as an indicative theoretical uncertainty.

The factorization scheme serves as a method to systematically eliminate collinear divergencies from $\hat{\sigma}_{i j}$ and absorb them into the PDFs which are extracted from global fits to measurements of deep inelastic scattering experiments where either electrons, positrons or neutrinos collide with nucleons [81, 82, 83. Different groups of physicists have derived parametrizations of proton PDFs from experimental data [84, 85, 86, 87]. As an example Fig. 5(a) shows PDFs of the CTEQ collaboration [84]. For antiprotons the distributions in Fig. 馬(a) have to be reversed between quark and antiquark. The gluons start to dominate in the $x$ region below 0.15. At the TEVATRON, the $t \bar{t}$ production cross-section is driven by the large $x$ region, since $m_{t}$ is relatively large compared to the TEVATRON beam energy $\left(m_{t} / \sqrt{s}=0.0875\right)$. At the LHC $\left(m_{t} / \sqrt{s}=0.0125\right)$ the lower $x$ region becomes more important. To 


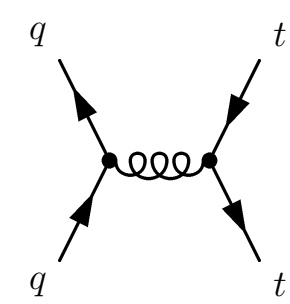

(a)

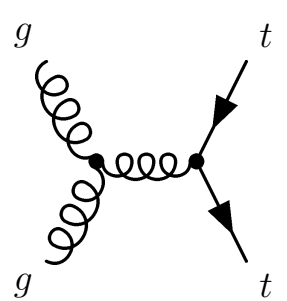

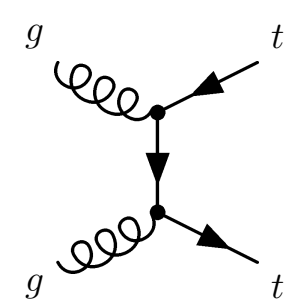

(b)

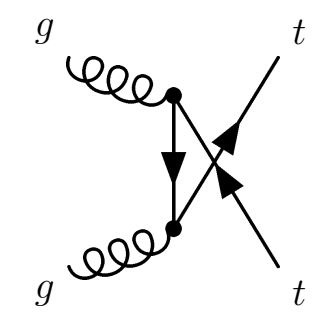

.

Figure 6: Feynman diagrams of the LO processes for $t \bar{t}$ production: (a) quark-antiquark annihilation $(q \bar{q} \rightarrow t \bar{t})$ and (b) gluon-gluon fusion $(g g \rightarrow t \bar{t})$.

compare different collider setups it is instructive to compute parton luminosities

$$
L_{i j}\left(\hat{s} ; s, \mu_{F}\right)=\frac{1}{s} \int_{\hat{s}}^{s} f_{i / A}\left(\frac{\tilde{s}}{s}\right) f_{j / B}\left(\frac{\hat{s}}{\tilde{s}}\right) \frac{1}{\tilde{s}} \mathrm{~d} \tilde{s} .
$$

The corresponding functions for the Tevatron and the LHC are shown in Fig. 或(b) 88. The increase of reach in $\sqrt{\hat{s}}$ is apparent. In addition to that advantage, the LHC in its final stage will have a luminosity which is 100 times larger than the one at the TEvatron. It is also interesting to note that at the LHC the $q g$ luminosity will dominate, while at the TEVATRON the $q \bar{q}$ luminosity is the largest one.

\subsection{Top-Antitop-Quark Pair Production}

The cross section $\hat{\sigma}$ of the hard parton-parton process $i j \rightarrow t \bar{t}$ can be calculated in perturbative QCD. The leading order (LO) processes, contributing with $\alpha_{s}^{2}$ to the perturbation series, are quark-antiquark annihilation, $q \bar{q} \rightarrow t \bar{t}$, and gluon-gluon fusion, $g g \rightarrow t \bar{t}$. The corresponding Feynman diagrams for these processes are depicted in Fig. 6. The LO differential cross section for $t \bar{t}$ production via $q \bar{q}$ annihilation is given by [89]

$$
\frac{\mathrm{d} \hat{\sigma}}{\mathrm{d} \hat{t}}(q \bar{q} \rightarrow t \bar{t})=\frac{4 \pi \alpha_{s}^{2}}{9 \hat{s}^{4}} \cdot\left[\left(m_{t}^{2}-\hat{t}\right)^{2}+\left(m_{t}^{2}-\hat{u}\right)^{2}+2 m_{t}^{2} \hat{s}\right]
$$

where $\hat{s}, \hat{t}$ and $\hat{u}$ are the Lorentz-invariant Mandelstam variables of the partonic process. They are defined by $\hat{s}=\left(p_{q}+p_{\bar{q}}\right)^{2}, \hat{t}=\left(p_{q}-p_{t}\right)^{2}$ and $\hat{u}=\left(p_{q}-p_{\bar{t}}\right)^{2}$ with $p_{i}$ being the corresponding momentum four-vector of the quark $i$, while $m_{t}$ denotes the top-quark mass.

A full calculation of next-to-leading order (NLO) corrections contributing in order $\alpha_{s}^{3}$ to the inclusive parton-parton cross-section for heavy quark pair-production was performed in the late 1980s and early 1990s [90, 91]. The NLO calculations involve virtual contributions to the LO processes, gluon bremsstrahlung processes $(q \bar{q} \rightarrow t \bar{t}+g$ and $g g \rightarrow t \bar{t}+g)$ as well as flavor excitation processes like $g+q(\bar{q}) \rightarrow t \bar{t}+q(\bar{q})$. For the NLO calculation of the total hadron-hadron cross section $\sigma(A B \rightarrow t \bar{t})$ to be consistent, one has to use NLO determinations of the coupling constant $\alpha_{s}$ and the PDFs. All quantities have to be defined in the same renormalization and factorization scheme as different approaches distribute radiative corrections differently among $\hat{\sigma}(t \bar{t})$, the PDFs and $\alpha_{s}$. Most authors use the $\overline{\mathrm{MS}}$ (modified minimal subtraction) scheme [2] or an extension of the latter. Corrections at NLO including full spin information are also available [93]. Recently, analytic expressions for the NLO QCD corrections to inclusive $t \bar{t}$ production were derived [94].

At energies close to the kinematic threshold, $\hat{s}=4 m_{t}^{2}, q \bar{q}$ annihilation is the dominant process, if the incoming quarks are valence quarks, as is the case of $p \bar{p}$ collisions. At the Tevatron, about $85 \%$ of $\sigma(t \bar{t})$ is due to $q \bar{q}$ annihilation [95]. At higher energies, the $g g$-fusion process dominates for both $p \bar{p}$ and $p p$ collisions, see also Fig. [5] (b). That is why, one can built the LHC as a $p p$ machine without 
Table 2: Cross-section predictions for $t \bar{t}$ production at approximate NNLO in perturbation theory including the resummation of initial-state gluon-bremsstrahlung. The cross sections are given for $p \bar{p}$ collisions at the Tevatron $(\sqrt{s}=1.8 \mathrm{TeV}$ [95] and $\sqrt{s}=1.96 \mathrm{TeV}$ [100]) and $p p$ collisions at the LHC [100], assuming $m_{t}=175 \mathrm{GeV} / c^{2}$. To derive the central values, the factorization and renormalization scale is set to $\mu=m_{t}$. The PDF parametrization CTEQ6.6 is used.

\begin{tabular}{ccc}
\hline Initial State & $\sqrt{s}$ & $\sigma(t \bar{t})$ \\
\hline$p \bar{p}$ & $1.8 \mathrm{TeV}$ & $5.19_{-0.68}^{+0.52} \mathrm{pb}$ \\
$p \bar{p}$ & $1.96 \mathrm{TeV}$ & $6.90_{-0.64}^{+0.46} \mathrm{pb}$ \\
$p p$ & $10 \mathrm{TeV}$ & $374_{-33}^{+18} \mathrm{pb}$ \\
$p p$ & $14 \mathrm{TeV}$ & $827_{-63}^{+27} \mathrm{pb}$ \\
\hline
\end{tabular}

compromising the production cross-section. Technically, it is of course much easier to operate a $p p$ collider, since one spares the major challenge to produce high antiproton currents in a storage ring. For the Tevatron, the ratio of NLO over LO cross-sections for $g g$ fusion is predicted to be 1.8 at $m_{t}=175 \mathrm{GeV} / c^{2}$, for $q \bar{q}$ annihilation the value is only about 1.2 [96]. Since the annihilation process is dominating, the overall NLO enhancement is about 1.25 .

Contributions to $\sigma(t \bar{t})$ due to radiative corrections are large in the region near threshold $\left(\hat{s}=4 m_{t}^{2}\right)$ and at high energies $\left(\hat{s}>400 m_{t}^{2}\right)$. Near threshold the cross section is enhanced due to initial state gluon bremsstrahlung [97]. This effect is important at the TEvatron, but less relevant for the LHC where gluon splitting and flavour excitation are increasingly important effects. The calculation at fixed NLO accuracy has been refined to systematically incorporate higher order corrections due to soft gluon radiation [88, 98, 99]. Technically, this is done by applying an integral transform (Mellin transform) to the cross section: $\sigma_{N}(t \bar{t}) \equiv \int_{0}^{1} \rho^{N-1} \sigma(\rho ; t \bar{t}) \mathrm{d} \rho$, where $\rho=4 m_{t}^{2} / s$ is a dimensionless parameter. In Mellin moment space the corrections due to soft gluon radiation are given by a power series of logarithms $\ln N$. For $\mu=m_{t}$ the corrections are positive at all orders. Therefore, the resummation of the soft gluon logarithms yields an increase of $\sigma(t \bar{t})$ with respect to the NLO value. Calculations by different groups implementing the resummation approach [88, 98, 99] are in good agreement. Exemplarily, the results of [95, 100] are quoted in Table 2, The quoted uncertainties include the uncertainty due to the choice of $\mu_{\mathrm{F}}$ and $\mu_{\mathrm{R}}$, and the uncertainty associated with the PDF parametrization.

The cross-section predictions strongly depend on $m_{t}$, which is illustrated in Fig. 7 that shows the predictions for the TEVATRON in comparison to measurements by CDF [101] and DØ [102]. Within the uncertainties the measurements agree well with the theoretical predictions.

\subsection{Single Top-Quark Production}

Top quarks can be produced singly via electroweak interactions involving the $W t b$ vertex. There are three production modes which are distinguished by the virtuality $Q^{2}$ of the $W$ boson $\left(Q^{2}=-q^{2}\right.$, where $q$ is the four-momentum of the $W$ ):

1. the t channel $\left(q^{2}=\hat{t}\right)$ : A virtual $W$ boson strikes a $b$ quark (a sea quark) inside the proton. The $W$ boson is spacelike $\left(q^{2}<0\right)$. This mode is also known as $W g$ fusion, since the $b$ quark originates from a gluon splitting into a $b \bar{b}$ pair. Another name used for this process is tq $\bar{b}$ production. Feynman diagrams representing this process are shown in Fig. \&[(a) and Fig. \&[(b). Production in the $t$ channel is the dominant source of single top quarks at the TEVATRON and at the LHC.

2. the s channel $\left(q^{2}=\hat{s}\right)$ : This production mode is of Drell-Yan type and is also called $t \bar{b}$ produc- 


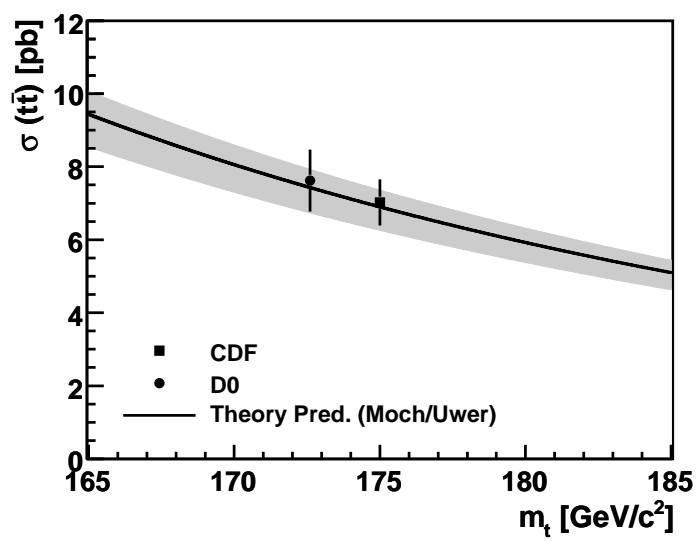

Figure 7: The $t \bar{t}$ cross ection in $p \bar{p}$ collisions at $\sqrt{s}=1.96 \mathrm{TeV}$ as a function of $m_{t}$. The measurements by CDF [101] and DØ [102] are compared to the theory prediction [100].

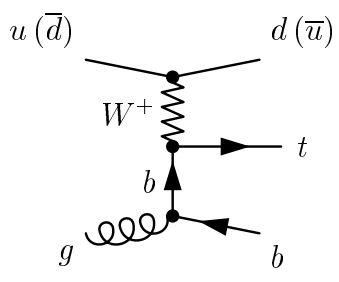

(a)

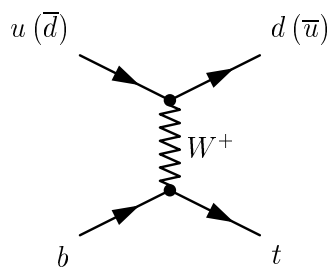

(b)

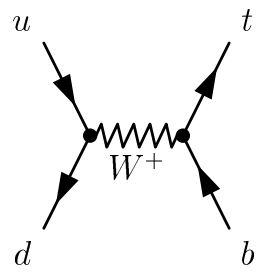

(c)

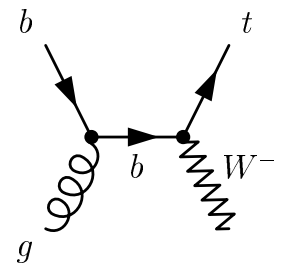

(d)

Figure 8: Representative Feynman diagrams for the three single top-quark production modes. (a) and (b) show $W g$-fusion graphs, (c) the $s$-channel process, and (d) associated production. The graphs show single top-quark production, the diagrams for single antitop-quark production can be obtained by interchanging quarks and antiquarks.

tion. A timelike $W$ boson with $q^{2} \geq\left(m_{t}+m_{b}\right)^{2}$ is produced by the fusion of two quarks belonging to an $\mathrm{SU}(2)$-isospin doublet. See Fig. $8(\mathrm{c})$ for the Feynman diagram.

3. associated production: The top quark is produced in association with a real (or close to real) $W$ boson $\left(q^{2}=M_{W}^{2}\right)$. The initial $b$ quark is a sea quark inside the proton. Fig. \& $8(\mathrm{~d})$ shows the Feynman diagram. The cross section is negligible at the TEvatron, but of considerable size at LHC energies where associated $W t$ production even supercedes the $s$ channel.

In $p \bar{p}$ and $p p$ collisions the cross section is dominated by contributions from $u$ and $d$ quarks coupling to the $W$ boson on one hand side of the Feynman diagrams. There is also a small contribution from the second weak-isospin quark doublet $(c, s)$; an effect of about $2 \%$ for $s$ - and $6 \%$ for $t$-channel production [103]. In the following, we will only consider single top-quark production via a $W t b$ vertex, since the production channels involving $W t d$ or $W t s$ vertices are strongly suppressed due to small CKM matrix elements. Assuming unitarity of the three-generation CKM matrix one obtains $\left|V_{t d}\right|=8.74_{-0.37}^{+0.26} \cdot 10^{-3}$ and $\left|V_{t s}\right|=(40.7 \pm 1.0) \cdot 10^{-3}[11]$. In the following paragraphs the status of theoretical cross-section predictions for the three single top-quark processes is reviewed.

t-channel Production The $t$-channel process was first suggested as a potentially interesting source of top quarks in the mid 1980s [104, 105] and early 1990s [106]. If the $b$ quark is taken to be massless, a singularity arises, when computing the diagram in Fig. \&](a) in case the final $\bar{b}$ quark is collinear with the incoming gluon. In reality, the non-zero mass of the $b$ quark regulates this collinear divergence. 
Table 3: Predicted total cross-sections for single top-quark production-processes. The cross sections are given for $p \bar{p}$ collisions at the Tevatron $(\sqrt{s}=1.96 \mathrm{TeV})$ and $p p$ collisions at the LHC $(\sqrt{s}=$ $14 \mathrm{TeV}$ ). The cross sections of the $t$-channel process are taken from [108, 109] and are evaluated with CTEQ5M1 PDFs. The values for $s$-channel and associated production are taken from [111, 112] using the MRST2004 NNLO PDFs. All cross sections are evaluated at $m_{t}=175 \mathrm{GeV} / c^{2}$.

\begin{tabular}{lcccc}
\hline Process & $\sqrt{s}$ & $\sigma_{t q \bar{b}}$ & $\sigma_{t \bar{b}}$ & $\sigma_{W t}$ \\
\hline$p \bar{p} \rightarrow t / \bar{t}$ & $1.96 \mathrm{TeV}$ & $1.98_{-0.22}^{+0.28} \mathrm{pb}$ & $1.02 \pm 0.08 \mathrm{pb}$ & $0.25 \pm 0.03 \mathrm{pb}$ \\
\hline$p p \rightarrow t$ & $14.0 \mathrm{TeV}$ & $156 \pm 8 \mathrm{pb}$ & $7.23_{-0.47}^{+0.55} \mathrm{pb}$ & $41.1 \pm 4.2 \mathrm{pb}$ \\
$p p \rightarrow \bar{t}$ & $14.0 \mathrm{TeV}$ & $91 \pm 5 \mathrm{pb}$ & $4.03_{-0.16}^{+0.14} \mathrm{pb}$ & $41.1 \pm 4.2 \mathrm{pb}$ \\
\hline
\end{tabular}

When calculating the total cross section, the collinear singularity manifests itself as terms proportional to $\ln \left(\left(Q^{2}+m_{t}^{2}\right) / m_{b}^{2}\right)$, causing the perturbation series to converge rather slowly. This difficulty can be obviated by introducing a PDF for the $b$ quark, $b\left(x, \mu^{2}\right)$, which effectively resums the logarithms to all orders of perturbation theory and implicitly describes the splitting of gluons into $b \bar{b}$ pairs inside the colliding hadrons [107]. Once a $b$-quark PDF is introduced into the calculation, the LO process is $q_{i}+b \rightarrow q_{j}+t$ as shown in Fig. \&(b). In this formalism the process shown in Fig. \&(a) is a higher order correction which is already partially included in the $b$-quark PDF. The remaining contribution is of order $1 / \ln \left(\left(Q^{2}+m_{t}^{2}\right) / m_{b}^{2}\right)$ with respect to the LO process. Additionally, there are also corrections of order $\alpha_{s}$. Cross-section calculations exist at NLO [108, 109, 110] and are summarised in Table 3. The cross sections given for the TEVATRON are the sum of top-quark and antitop-quark production. In $p p$ collisions at the LHC the $t$-channel cross section $\sigma_{t q \bar{b}}$ differs for top-quark and antitop-quark production, which are therefore treated separately in Table 3. The contribution of soft-gluon corrections near the partonic threshold has been investigated, but was found to be small at the TEvatron [111]. At the LHC, the resummation approach is not meaningful, when calculating $\sigma_{t q \bar{b}}$, since threshold corrections do not dominate the perturbative expansion [112].

The ratio of $\sigma_{t q \bar{b}} / \sigma_{t \bar{t}}$ is about $30 \%$, for the TEVATRON as well as for the LHC. The uncertainties quoted in Table 3 include the uncertainties due to the factorization scale $\mu$, the choice of PDF parameterization, and the uncertainty in $m_{t}$. The factorization scale uncertainty is $\pm 4 \%$ at the TEVATRON and $\pm 3 \%$ at the LHC. The central value was calculated with $\mu^{2}=Q^{2}+m_{t}^{2}$ for the $b$-quark PDF. The scale for the light-quark PDFs was set to $\mu^{2}=Q^{2}$. The dependence of the $t$-channel single top-quark cross-section is approximately linear in the relevant mass range, with a slope of $-1.6 \% \cdot \sigma_{t q \bar{b}} / 1 \mathrm{GeV} / c^{2}$ at the TEVATRON and $-0.7 \% \cdot \sigma_{t q \bar{b}} / 1 \mathrm{GeV} / c^{2}$ at the LHC.

An interesting feature of single top-quark production (in the $t$-channel and in the $s$-channel) is that in its rest frame the top quark is $100 \%$ polarized along the direction of the $d$ quark ( $\bar{d}$ quark) [113, 114, 103, 115]. The reason for this is that the $W$ boson couples only to fermions with left-handed chirality. Consequently, the ideal basis to study the top-quark spin is the one which uses the direction of the $d$ quark as the spin axis [114. In $p \bar{p}$ collisions at the TEVATRON $t$-channel single top-quark production proceeds via $u g \rightarrow t d \bar{b}$ in about $77 \%$ of the cases. The $d$ quark can then be measured by the light-quark jet in the event. The top-quark spin is best analyzed in the spectator basis for which the spin axis is defined along the light-quark jet-direction. However, $23 \%$ of the events proceed via $\bar{d} g \rightarrow t \bar{u} \bar{b}$, in which case the $\bar{d}$ quark is moving along one of the beam directions. For these events the spectator basis is not ideal, but since the light-quark jet occurs typically at high rapidity the dilution is small. In total, the top quark has a net spin polarization of $96 \%$ along the direction of the light-quark jet in $t q \bar{b}$ production at the TEvatron [114]. In $s$-channel events the best choice is the antiproton-beam direction as spin basis, leading to a net polarization of $98 \%$ [114]. 
Since the top quark does not hadronize, its decay products carry information about the top-quark polarization. A suitable variable to investigate the top-quark polarization is the angular distribution of electrons and muons originating from the decay chain $t \rightarrow W^{+} b, W^{+} \rightarrow \ell^{+} \nu_{\ell}$. If $\theta_{q \ell}$ is the angle between the charged lepton and the light-quark jet-axis in the top-quark rest-frame, the angular distribution is given by $d N / d \cos \theta_{q \ell}=\frac{1}{2}\left(1+P \cos \theta_{q \ell}\right)$ [116] with $P=1$ corresponding to fully polarized top quarks.

s-channel Production The $s$-channel-production mode of single top-quarks ( $t \bar{b}$ production) probes a complementary aspect of the weak charged-current interaction of the top quark, since it is mediated by a timelike $W$ boson as opposed to a spacelike $W$ boson in the $t$-channel process. The leading order $s$-channel process is depicted in Fig. E(c). Some first order $\left(\alpha_{s}\right)$ corrections have the same initial and final states as the $t$-channel diagrams shown in Fig. \& (a). However, these two classes of diagrams do not interfere because they have a different colour structure. The $t \bar{b}$ pair in the $t$-channel process is in a colour-octet state, since it originates from a gluon. In $s$-channel production the $t \bar{b}$ pair forms a colour-singlet because it comes from a $W$. The different colour structure implies that both groups of processes must be separately gauge invariant and, therefore, they do not interfere [104, 106, 103].

Cross sections for $t \bar{b}$ production are available at NLO including the resummation of soft gluon corrections [111, 112, see Table 3. The predictions for the $s$-channel cross section have a smaller uncertainty from the PDFs than for the $t$-channel because they do not depend as strongly on the gluon PDF as the $t$-channel calculation does. The uncertainty in $m_{t}$ leads to an uncertainty in the cross section of about $6 \%\left(\Delta m=2.1 \mathrm{GeV} / c^{2}\right)$.

The ratio of cross sections for the $t$-channel and $s$-channel mode is 1.8 at the TEVATRON $(\sqrt{s}=$ $1.96 \mathrm{TeV}$ ) and 22 at the LHC. In $p p$ collisions the gluon-initiated processes, $t \bar{t}$ production and $t$-channel single top-quark production, dominate by far over $s$-channel single top-quark production which is a $q \bar{q}$-annihilation process.

Wt Production The third single top-quark production mode is characterised by the associated production of a top quark and an on-shell (or close to on-shell) $W$ boson. Cross-section predictions are given in Table 3. It is obvious that $W t$ production is negligible at the TEvaTron, but is quite important at the LHC where it even exceeds the $s$-channel production-rate. The errors quoted in Table 3 include the uncertainty due to the choice of the factorization scale $( \pm 10 \%$ at the LHC) and the PDFs $( \pm 2.5 \%$ at the LHC). The uncertainty in $m_{t}\left(\Delta m=2.1 \mathrm{GeV} / c^{2}\right)$ causes a spread of the cross section by $\pm 3.5 \%$. At NLO, corrections to the $W t$ cross section arise that correspond to LO $g g \rightarrow t \bar{t}$ production with one subsequent top-quark decay. When calculating the cross section one has to account for that to avoid double counting [117, 118]. First studies [79, 119] show that $W t$ production will be observable at the LHC with data corresponding to about $10 \mathrm{fb}^{-1}$ of integrated luminosity.

\subsection{Top-Quark Decay}

Within the SM, top quarks decay predominantly into a $b$ quark and a $W$ boson, while the decays $t \rightarrow d+W^{+}$and $t \rightarrow s+W^{+}$are strongly CKM-suppressed and are therefore neglected in the following discussion. The top-quark decay rate, including first order QCD corrections, is given by

$$
\Gamma_{t}=\frac{G_{F} m_{t}^{3}}{8 \pi \sqrt{2}}\left|V_{t b}\right|^{2}\left(1-\frac{M_{W}^{2}}{m_{t}^{2}}\right)^{2}\left(1+2 \frac{M_{W}^{2}}{m_{t}^{2}}\right)\left[1-\frac{2 \alpha_{s}}{3 \pi} \cdot f(y)\right]
$$

with $y=\left(M_{W} / m_{t}\right)^{2}$ and $f(y)=2 \pi^{2} / 3-2.5-3 y+4.5 y^{2}-3 y^{2} \ln y$ [120, 121, 122]. The QCD corrections of order $\alpha_{s}$ lower the LO decay rate by $-10 \%$, yielding $\Gamma_{t}=1.34 \mathrm{GeV}$ at $m_{t}=172.6 \mathrm{GeV} / c^{2}$. The decay width for events with hard gluon radiation $\left(E_{g}>20 \mathrm{GeV}\right)$ in the final state has been estimated to be $5-10 \%$ of $\Gamma_{\text {top }}$, depending on the gluon jet definition (cone size $\Delta R=0.5$ to 1.0) [123]. Electroweak 
corrections to $\Gamma_{\text {top }}$ increase the decay width by $\delta_{\mathrm{EW}}=+1.7 \%$ [124, 125]. Taking the finite width of the $W$ boson into account leads to a negative correction $\delta_{\Gamma}=-1.5 \%$ such that $\delta_{\mathrm{EW}}$ and $\delta_{\Gamma}$ almost cancel each other 126 .

The large $\Gamma_{t}$ implies a very short lifetime of $\tau_{t}=1 / \Gamma_{t} \approx 5 \cdot 10^{-25} \mathrm{~s}$ which is smaller than the characteristic formation time of hadrons $\tau_{\text {form }} \approx 1 \mathrm{fm} / c \approx 3 \cdot 10^{-24} \mathrm{~s}$. In other words, top quarks decay before they can couple to light quarks and form hadrons. The lifetime of $t \bar{t}$ bound states, toponium, is too small, $\Gamma_{t \bar{t}} \sim 2 \Gamma_{t}$, to allow for a proper definition of a bound state with sharp binding energy, a feature already pointed out in the early 1980s [127].

The decay amplitude is dominated by the contribution from longitudinal $W$ bosons because the decay rate of the longitudinal component scales with $m_{t}^{3}$, while the decay rate into transverse $W$ bosons increases only linearly with $m_{t}$. In both cases the $W^{+}$couples solely to $b$ quarks of left-handed chirality, which translates into left-handed helicity, since the $b$ quark is effectively massless compared to the energy scale set by $m_{t}$. If the $b$ quark is emitted antiparallel to the top-quark spin axis, the $W^{+}$must be longitudinally polarized, $h^{W}=0$, to conserve angular momentum. If the $b$ quark is emitted parallel to the top-quark spin axis, the $W^{+}$boson has helicity $h^{W}=-1$ and is transversely polarized. $W$ bosons with positive helicity are thus forbidden in top-quark decays due to angular momentum conservation, assuming $m_{b}=0$. The ratios of decay rates into the three $W$ helicity-states are given by [120]:

$$
\Gamma\left(h^{W}=-1\right): \Gamma\left(h^{W}=0\right): \Gamma\left(h^{W}=+1\right)=1: \frac{m_{t}^{2}}{2 M_{W}^{2}}: 0 .
$$

In the decay of antitop quarks negative helicity is forbidden. Strong NLO corrections to the decay-rate ratios lower the fraction of longitudinal $W$ bosons by $1.1 \%$ and increase the fraction of left-handed $W$ bosons by $2.2 \%$ [128, 129]. Electroweak and finite widths effects have even smaller effects on the helicity ratios, inducing corrections at the per-mille level [130]. Taking the non-zero b-quark mass into account yields very small corrections, leading to a non-zero fraction of $W$ bosons with positive helicity $\Gamma_{+} / \Gamma=3.6 \cdot 10^{-4}$ at Born level [128].

The spin orientation (helicity) of $W$ bosons from top-quark decays is propagated to its decay products. In case of leptonic $W$ decays the polarization is preserved and can be measured [131]. The angular distribution of charged leptons from $W$ decays originating from top quarks is given by

$$
\frac{1}{\Gamma} \frac{\mathrm{d} \Gamma}{\mathrm{d} \cos \theta_{\ell}}=\frac{3}{4} \frac{m_{t}^{2} \sin ^{2} \theta_{\ell}+M_{W}^{2}\left(1-\cos \theta_{\ell}\right)^{2}}{m_{t}^{2}+2 M_{W}^{2}}
$$

where $\pi-\theta_{\ell}$ is the angle between the $b$ quark direction and the charged lepton in the $W$ boson rest frame [132].

Given that the top quark decays almost $100 \%$ of the time as $t \rightarrow W b$, typical final states for the leading pair-production process can therefore be divided into three classes:

$$
\begin{aligned}
& \text { A. } \quad t \bar{t} \rightarrow W^{+} b W^{-\bar{b}} \rightarrow q \bar{q}^{\prime} b q^{\prime \prime} \bar{q}^{\prime \prime \prime} \bar{b}, \\
& \text { B. } \quad t \bar{t} \rightarrow W^{+} b W^{-} \bar{b} \rightarrow q \bar{q}^{\prime} b \ell \bar{\nu}_{\ell} \bar{b}+\bar{\ell} \nu_{\ell} b q \bar{q}^{\prime} \bar{b}, \\
& \text { C. } \quad t \bar{t} \rightarrow W^{+} b W^{-} \bar{b} \rightarrow \bar{\ell} \nu_{\ell} b \ell^{\prime} \bar{\nu}_{\ell^{\prime}} \bar{b},
\end{aligned}
$$

The quarks in the final state evolve into jets of hadrons. A, B, and $\mathrm{C}$ are referred to as the all-jets, lepton + jets $(\ell+$ jets $)$, and dilepton $(\ell \ell)$ channels, respectively. The relative contribution of the three channels A, B, C, including hadronic corrections, are given in parentheses above. While $\ell$ in the above processes refers to $e, \mu$, or $\tau$, most of the results to date rely on the $e$ and $\mu$ channels. Therefore, in what follows, $\ell$ will be used to refer to $e$ or $\mu$, unless noted otherwise. 


\section{Hadron Colliders and Collider Detectors}

The first hadron collider, the ISR at CERN, had to overcome two initial obstacles. The first was low luminosity, which steadily improved over time. The second was the broad angular spread of interesting events for which it was noted [133] that one needs " sophisticated detectors covering at least the whole central region $\left(45^{\circ}<\theta<135^{\circ}\right)$ and full azimuth". This statement reflects the major revelation of the period, namely that hadrons have partonic substructure producing a strong hadronic yield at large transverse momentum $\left(p_{T}\right)$. Caught unprepared, the ISR missed the discovery of the $\mathrm{J} / \psi$ and later missed the $\Upsilon$, though it did make important contributions in other areas and paved the way for future hadron colliders. The partonic substructure of colliding hadrons leads to multiple production mechanisms, $\left(q \bar{q}, q q^{\prime}, q g, g g \ldots\right)$ and a broad range of center of mass (CM) energies that allow one to probe mass scales far above or below the average constituent CM energy. A side effect is the absence of a beam energy constraint so that event momentum and energy generally only balance in the plane transverse to the beam axis. Another aspect of hadron collisions is the fact that cross-sections for interesting processes are many orders of magnitude below the total inelastic cross-section $\left(\sigma_{\text {tot }}\right)$. At the Fermilab Tevatron the value of $\sigma_{\text {tot }}$ is $\sim 60 \mathrm{mb}$ and rises to $\sim 100 \mathrm{mb}$ at the LHC, where standard model (SM) Higgs could have a production cross-section of order $10^{-10} \times \sigma_{\text {tot }}$ or smaller, depending on $M_{H}$. High luminosities also mean multiple interactions per beam crossing. Hadron beams are segmented into bunches, each containing $10^{11}-10^{12}$ protons or anti-protons. Tevatron bunches are separated in time by $396 \mathrm{~ns}$ for a bunch crossing rate of $2.5 \mathrm{MHz}$. The Tevatron currently attains peak luminosities in excess of $\mathcal{L}=3.5 \times 10^{32} \mathrm{~cm}^{-2} s^{-1}$ with an average of $10 p \bar{p}$ interactions per crossing. The LHC will have a bunch spacing of as little as $25 \mathrm{~ns}$ for a crossing rate of $40 \mathrm{MHz}$. There are expected to be an average of $\sim 25$ interactions per crossing at the design luminosity of $\mathcal{L}=10^{34} \mathrm{~cm}^{-2} \mathrm{~s}^{-1}$, corresponding to the production of as many as $10^{4}$ particles every $100 \mathrm{~ns}$.

In spite of a very challenging environment, hadron collider experiments have produced new discoveries as well as precision measurements. The cross-section for $\mathrm{W}$ production at the CERN SPS was $10^{-8} \times \sigma_{\text {tot }}$ at the time of its discovery [134, 135] and hadron collider experiments have since measured the $\mathrm{W}$ mass to high precision: $M_{W}(C D F \oplus D 0)=80.432 \pm 0.039 \mathrm{GeV} / \mathrm{c}^{2}$ [136]. Similarly, $t \bar{t}$ production was observed at $10^{-10} \times \sigma_{\text {tot }}$ at the Fermilab Tevatron [46, 47] where $M_{t}$ has since been measured to an accuracy of better than 1\% [50], as discussed below. For the discovery of $t \bar{t}$ production, hermetic calorimetry and very good lepton identification enabled signal events to be efficiently accumulated and reconstructed and a variety of techniques were used to distinguish signal from backgrounds. Though there was considerable skepticism about the utility of silicon microstrip detectors in a hadron collider environment [137], CDF silicon detectors [138, 139] dispelled all doubts, providing high precision tracking that made it possible to select extremely pure event samples by reconstructing the displaced vertices of $b$ hadron decays.

At present, the Tevatron at FNAL and the Large Hadron Collider (LHC) at CERN are the only machines capable of $t \bar{t}$ production. The collider detectors associated with these machines are often compared to cameras, recording a picture each time the beams collide at their centers. There is some truth to this analogy, but in reality the collisions occur so frequently that there is initially only enough time to draw a rough picture of what happened. If this picture gives an indication that something interesting has happened, a sequence of actions is then "triggered" that results in successively clearer pictures of the collision, each scrutinized to a greater extent. If a collision manages to remain interesting until the completion of this sequence, it is written to storage media. Typically only a few hundred collisions out of every 2 - 40 million occurring each second are recorded for posterity. This is a very important aspect of hadron colliders. The "trigger" system that decides whether or not to record data is the first and most critical step of the analysis of data. Furthermore, the tiny fraction of all collisions that are recorded still represent an enormous amount of data. Hadron colliders can easily acquire more than a petabyte $\left(10^{15}\right.$ bytes $)$ of raw data per year. 
Though different in their details, the hadron collider detectors at the Tevatron and LHC are similar in most respects. Primary $p \bar{p}(p p)$ interactions have a roughly Gaussian probability distribution along the beam-line, peaking at the detector center with $\sigma \sim 25 \mathrm{~cm}(\sim 5 \mathrm{~cm})$ at the Tevatron (LHC). In all cases, the central detector regions are cylindrical with their axes oriented along the horizontal direction. The cylinders are sandwiched between endcap regions arrayed as planar disks. Though it was not the case in previous generations of collider detectors at the Tevatron and SPS collider, all detectors now include a magnetic spectrometer, with the tracking system surrounded by calorimetry and muon chambers. Electrons, photons, and hadronic jets are identified using calorimeters and tracking while muons are identified by muon stations and tracking. The data are collected using a multi-level trigger system. The coordinate system used with hadron collider detectors is right-handed with the $\mathrm{z}$ axis along the direction of one of the beams, the $\mathrm{y}$ axis vertical and the $\mathrm{x}$ axis in the plane of the accelerator. The coordinates are also expressed in relation to particle trajectories via azimuthal angle $\phi$, rapidity $y$, and pseudorapidity $\eta$. The latter are functions of the polar angle $\theta$ and particle velocity $\beta: y(\theta, \beta) \equiv \frac{1}{2} \ln [(1+\beta \cos \theta) /(1-\beta \cos \theta)]$, and $\eta(\theta) \equiv y(\theta, \beta=1)$.

In the remainder of this section, essential features of the Tevatron and LHC, the general purpose detectors that record the remnants of hadron collisions from these machines and the basic methods used to select and reconstruct $t \bar{t}$ events are presented.

\subsection{Collider Experiments at the Tevatron in Run 2 (2001-present)}

Upgrades to the FNAL accelerator complex, including the addition of the new Main Injector, electron cooling, and the anti-proton recycler have enabled a luminosity increase of more than one order of magnitude relative to Run 1 (1992-1996). In addition, the superconducting magnets used in Run 2 are operated at a lower temperature allowing margin for higher currents to produce higher fields, supporting an increase in CM energy from $1.8 \mathrm{TeV}$ to $1.96 \mathrm{TeV}$. In Run 1 , luminosity peaked at $\mathcal{L} \sim 3 \times 10^{31} \mathrm{~cm}^{-2} \mathrm{~s}^{-1}$ with bunch spacing of $\sim 3.5 \mu \mathrm{s}$. In Run 2 peak luminosities are currently within about $35 \%$ of the Run 2 target of $\mathcal{L} \sim 5 \times 10^{32} \mathrm{~cm}^{-2} \mathrm{~s}^{-1}$. To date, the Tevatron has delivered over $6 \mathrm{fb}^{-1}$ to the CDF and D0 experiments and is adding new data at the rate of $\sim 2.5-3.0 \mathrm{fb}^{-1} /$ year. To handle the higher collision rates, the detectors were upgraded with new front-end and trigger electronics. Both CDF and D0 also installed completely new and more powerful tracking systems for Run 2 that include large-area, multi-layer silicon detectors.

The CDF Run 2 upgrade [140] includes silicon tracking, a wire drift chamber, a time-of-flight (TOF) detector, new endplug calorimeters, extended muon systems, and new front-end electronics, trigger, and data acquisition (DAQ). The goals of the 722,000 channel silicon system were to increase tracking coverage to $|\eta| \leq 2$, provide precise 2 dimensional (2D) and reasonable 3 dimensional (3D) impact parameter and vertex resolution and dead-time-less triggering on displaced tracks. The core of the system is the SVX II detector [140, 141] which has 5 double-sided layers extending to $\pm 45 \mathrm{~cm}$ from the center of the detector as necessitated by the broad span of interactions along the beam axis. The SVX II hit information is used in the online trigger [142] which makes it possible to collect large samples of hadronic charm and bottom particle decays as well as to tag long-lived particles in high $p_{T}$ events. Each of the 5 layers of SVX II contains double-sided Silicon that combines axially oriented strips with strips oriented either at $90^{\circ}$ or $1.2^{\circ}$ to the axial direction. The $1.2^{\circ}$ stereo angle provides less ambiguous matching to the axial strips but this is achieved at the cost of hit position resolution in the direction parallel to the strips. Typical hit resolutions in the $\mathrm{z}$ coordinate for correctly matched axial-stereo hit pairs are $\sim 1 \mathrm{~mm}(\sim 0.05 \mathrm{~mm})$ for $1.2^{\circ}\left(90^{\circ}\right)$ stereo angle. Inside the inner bore of the SVX II detector and mounted directly on the beam pipe in the radial range $1.35-1.70 \mathrm{~cm}$ is the Layer 00 silicon detector [143]. This layer uses single-sided $25 \mu \mathrm{m}$ pitch detectors with $50 \mu \mathrm{m}$ readout pitch. The readout electronics are mounted outside of the tracking volume. The silicon is cooled to $\sim 0^{\circ} \mathrm{C}$ and can operate at high bias voltages as necessitated by radiation damage. Layer 00 reduces uncertainty in 
the transverse impact parameter of tracks which in turn improves high $p_{T} b$ jet identification $(b$ tagging) and proper lifetime resolution in low $p_{T} b$ hadron decays. The SVT trigger and Layer 00 silicon were critical ingredients in the CDF observation of $B_{s}$ mixing [144]. Just outside the SVX II is the ISL [145] which is comprised of two layers of double-sided silicon $\left(1.2^{\circ}\right.$ stereo) at radii of 20 and $28 \mathrm{~cm}$. The ISL extends $\pm 95 \mathrm{~cm}$ along the beam axis. In the central region, $|\eta| \leq 1$, the ISL is surrounded by a wire drift chamber [146] with 96 wire planes grouped into eight superlayers, alternating between axial and $\pm 3^{\circ}$ stereo angle. Groups of 12 sense wires and 17 potential wires are contained in cells separated by $\mathrm{Au}-$ plated mylar sheets. Front-end electronics can measure charge deposition for particle identification. The tracking system has transverse momentum resolution $\sigma_{p_{T}} / p_{T}^{2} \sim 0.0017(\mathrm{GeV} / \mathrm{c})^{-1}$ and $r-\phi$ impact parameter resolution of $\sim 40 \mu \mathrm{m}$ which includes a $30 \mu \mathrm{m}$ contribution from the beamspot. Further particle identification is made possible by the TOF system [147] installed just beyond the drift chamber and constructed as a cylindrical array of scintillator bars. Scintillation light produced when a charged particle traverses the TOF is collected at both ends of the bars by fine-mesh photomultipliers. The time resolution is of order $120 \mathrm{ps}$ and enables kaon identification for $b$ flavor tagging. For improved electron and jet finding in the forward region, as well as improved missing transverse energy $\left(\mathbb{E}_{T}\right)$ resolution, CDF installed new scintillator-tile endplug calorimeters [148]. These have resolution comparable to the central calorimeters, namely $\sigma_{E} / E \sim 15 / \sqrt{E} \oplus 7 \%$ in the electromagnetic section which is designed to interact with and stop photons and electrons, and $\sigma_{E} / E \sim 70 / \sqrt{E} \oplus 4 \%$ in the hadronic section which is designed to interact with and stop hadrons. Outside the calorimeters, muon systems were upgraded to fill gaps and extend coverage. The CDF trigger system has 3 levels. The Level 1 hardware trigger has a pipeline of 42 cells allowing a $50 \mathrm{kHz}$ accept rate. At Level 2, there is a combination of hardware and firmware with a two stage asynchronous pipeline comprised of four buffer cells to allow an accept rate of $\sim 1 \mathrm{kHz}$. At Level 3 a Linux processor farm fully reconstructs events and selects $\sim 100$ events per second for storage.

The D0 Run 2 detector [149] includes a 793,000 channel Silicon Microstrip Tracker (SMT) [150] and a Central Fiber Tracker (CFT) inside a new $2 \mathrm{~T}$ solenoidal magnet with a radius of $60 \mathrm{~cm}$. The Run 2 upgrade also includes enhanced muon systems, and all new front-end electronics, trigger, and DAQ. The D0 silicon includes ' $F$ disks' which reside between and just beyond the barrels, and 'H disks' which are installed in the far forward regions. The barrels consist of axial and either $90^{\circ}$ or $2^{\circ}$ stereo layers while the $\mathrm{F}$ and $\mathrm{H}$ disks contain $\pm 15^{\circ}$ and $\pm 7.5^{\circ}$ symmetric $\mathrm{u}-\mathrm{v}$ doublets, respectively. Surrounding the silicon is the $2.6 \mathrm{~m}$ long CFT made up of eight layers of axial and $\pm 2^{\circ}$ stereo doublets of $1 \mathrm{~mm}$ diameter scintillating fibers. The 77,000 fibers are connected to visible light photon counters (VLPC) operated at $\sim 10^{\circ} \mathrm{C}$ with $\leq 0.1 \%$ noise occupancy. The axial layers are used in a Level 1 track trigger. The Silicon and Fibers are surrounded by the $2 \mathrm{~T}$ superconducting solenoidal magnet. The tracker has good momentum resolution and $r-\phi$ impact parameter resolution of $\sim 40 \mu m$ which includes a 30 $\mu m$ contribution from the beamspot. As for other experiments, the magnetic spectrometer can be used to improve the calibration of the calorimeter by comparing the transverse momenta of isolated tracks, (e.g. from electron candidates) with the corresponding transverse energy measurements.

In Run 2 D0 has retained its existing hermetic LAr calorimeter which has resolution $\sigma_{E} / E \sim$ $15 \% / \sqrt{E}$ for electrons and $\sigma_{E} / E \sim 50 \% / \sqrt{E}$ for pions. The total jet energy resolution is $\sim 15 \%$ at 30 $\mathrm{GeV}$ falling to $\sim 10 \%$ at $100 \mathrm{GeV}$ and to $\sim 5-7 \%$ above $200 \mathrm{GeV}$. There are also improved forward and central preshower detectors. The central preshower uses $7 \mathrm{~mm}$ prismatic scintillator strips to reduce the electron trigger rate by a factor of 5 . The D0 muon system is the outermost part of the detector and consists of a system of proportional drift tubes in the region $|\eta|<1$ and mini drift tubes that extend the coverage to $|\eta| \sim 2$. Scintillation counters are used for triggering and also for cosmic ray and beam-halo $\mu$ rejection. The system is completed by Toroidal magnets and special shielding. Each subsystem has 3 layers with the innermost layer in between the calorimeter and iron of the toroid. The average energy loss of $1.6 \mathrm{GeV}$ in the calorimeter and $1.7 \mathrm{GeV}$ in the iron is taken into account in determining the momenta of muons. The $p_{T}$ resolution of tracks from the SMT and CFT matched to $\mu$ 
segments is $\sigma_{p_{T}} / p_{T}=0.02 \oplus 0.002 p_{T} \mathrm{GeV} / \mathrm{c}$ [151]. The calorimeters use switched capacitor arrays for pipelining data. The silicon and fiber readout use the SVX2 chip which has a 32 cell pipeline. The multi-level trigger system focuses on regions of interest within events to avoid full event reconstruction. Level 0 is a beam crossing trigger ("minimum bias"). Level 1 is based upon calorimetry, preshower, fiber tracker, and muon scintillators as well as some information on displaced tracks from the silicon system and has a $2 \mathrm{kHz}$ accept rate. The level 2 hardware trigger has $1 \mathrm{kHz}$ accept rate. Level 3 is a processor farm with $50 \mathrm{~Hz}$ accept rate.

\subsection{Collider Detectors at the CERN LHC}

The LHC [152] will collide protons at $\sqrt{s}=10 \mathrm{TeV}$ in 2009 and 2010 with a bunch spacing of 50 ns. The luminosity will increase in tandem with increased understanding of the safe operation modes of the machine. Physics running will start at $\mathcal{L} \sim 10^{31} \mathrm{~cm}^{-2} \mathrm{~s}^{-1}$ and then increase in several steps to a target of $\mathcal{L} \sim 10^{32} \mathrm{~cm}^{-2} \mathrm{~s}^{-1}$. Of order $100 \mathrm{pb}^{-1}$ of data are planned to be delivered to each of the experiments. During a shutdown that is currently planned for 2011, additional protection systems will be installed in the LHC and the 1232 superconducting dipole magnets will be trained to operate at, or near to, the design current of $\sim 12 \mathrm{kA}$. Each dipole is $40 \mathrm{~m}$ long and operates at $1.9^{\circ} \mathrm{K}$. Subsequent running will be at or near $\sqrt{s}=14 \mathrm{TeV}$ with $25 \mathrm{~ns}$ bunch spacing and luminosity of $\mathcal{L}=\sim 10^{33} \mathrm{~cm}^{-2} \mathrm{~s}^{-1}$ for several years. The luminosity will then rise to $\mathcal{L} \sim 10^{34} \mathrm{~cm}^{-2} \mathrm{~s}^{-1}$ after exchanging Copper collimators for new ones made from Carbon.

ATLAS is the largest collider experiment ever built [153]. Its size is determined by the extent of its muon system which uses $20 \mathrm{~m}$ diameter air-core toroids and endcap planes separated by $46 \mathrm{~m}$. The ATLAS inner detector starts with pixel layers with $50 \mu \mathrm{m} \times 400 \mu \mathrm{m}$ pixels. There are three cylindrical layers at radii of $5.1,8.9$, and $12.3 \mathrm{~cm}$ and three end-cap disks per side. In total, the system contains $80 \mathrm{M}$ pixels. Outside the pixels there are four double-sided, shallow stereo barrel layers of silicon microstrips at radii of 30 to $51 \mathrm{~cm}$ and nine endcap disks per end. The total area of silicon is $\sim 61 \mathrm{~m}^{2}$. The Silicon is operated at -5 to $-10{ }^{\circ} \mathrm{C}$ to avoid destructive annealing that occurs after type inversion due to radiation exposure and which is accelerated at higher temperatures [154. Beyond the silicon is a transition-radiation, straw-tube tracker with 73 and 160 planes in the barrel and each end cap, respectively. Single hit resolution is $\sim 130 \mu \mathrm{m}$. The tracker will have momentum resolution of $\sigma_{p_{T}} / p_{T} \sim 5 \times 10^{-5} p_{T} \oplus 0.01\left(p_{T}\right.$ in $\left.\mathrm{GeV} / \mathrm{c}\right)$ with coverage for $|\eta| \leq 2.5$. The ATLAS tracker is situated in a $2 \mathrm{~T}$ solenoidal magnetic field. The calorimeter includes a $\mathrm{Pb}$-LAr accordion electromagnetic system in the central region surrounded by a scintillator-tile hadronic section. In the forward regions the electromagnetic and hadronic systems both use LAr. Preshower detectors are installed ahead of the electromagnetic calorimeter inside the solenoid cryostat wall and employ narrow strips to allow pointing to the interaction vertex with resolution of $\sim 50 \mathrm{mrad} / \sqrt{E}$. Overall the energy resolutions expected to be achieved are $\sigma(E) / E=10 / \sqrt{E} \oplus 0.2 \%$ with energy in $\mathrm{GeV}$ in the electromagnetic and $\sigma(E) / E \sim 50(80) / \sqrt{E} \oplus 3(6) \%$ in the central (forward) tile hadronic system with coverage of $|\eta| \leq 3.2$ (4.9) The muon system will provide momentum resolution of order $2-3 \%$ for intermediate momenta and $7-10 \%$ at $1 \mathrm{TeV}$ with coverage of $|\eta| \leq 2.7$. The ATLAS trigger has three levels. Level 1 is made up of custom electronics and uses coarse resolution data from the calorimeters and muon system. Level 2 is a software trigger run on a processor farm with full resolution data for regions associated with Level 1 trigger objects. The highest level is the Event Filter (EF) which has full resolution data for the full detector and runs full offline reconstruction and selection algorithms.

The CMS experiment [155] has an inner detector that includes a pixel detector with three barrel layers at radii between 4.4,7.3 and $10.2 \mathrm{~cm}$ and a silicon strip tracker with 10 barrel layers extending from a radius of $20 \mathrm{~cm}$ to a radius of $1.1 \mathrm{~m}$. Layers 1,2,5, and 6 of the silicon strip tracker are double-sided with $100 \mathrm{mrad}$ stereo angle. The size of the pixels is $100 \times 150 \mu \mathrm{m}^{2}$. Endcap systems consist of two disks in the pixel detector and three small inner disks plus nine large outer disks in the strip tracker on each side of 
the barrel. The silicon strip disks are composed of concentric rings of modules with ring 1 being closest to the beam. The innermost two of three rings in the small disks, and rings 1,2 and 5 of the large disks are double-sided. The disks extend tracking acceptance to $|\eta| \leq 2.5$. The CMS tracker is the largest silicon tracker ever built with more than $200 \mathrm{~m}^{2}$ of active silicon area. All told, there are $66 \mathrm{M}$ pixels and $9.6 \mathrm{M}$ silicon strips. The momentum resolution of the tracker is $\sigma_{p_{T}} / p_{T} \sim 1.0(2.0) \times 10^{-4} p_{T} \oplus 0.008(0.02)$ with $p_{T}$ in $\mathrm{GeV} / \mathrm{c}$ in the central (forward) regions. For muons, the additional lever arm of the muon system allows an improvement in resolution to $\sim 4.5 \% \times \sqrt{p_{T}(T e V)}$. The asymptotic transverse (longitudinal) impact parameter resolution is expected to be better than 35 (75) $\mu \mathrm{m}$. The CMS calorimeters include a $\mathrm{PbWO}_{4}$ crystal electromagnetic calorimeter with $\mathrm{Si}$ avalanche photodiode readout surrounded by a $\mathrm{Cu}$ and scintillator tile hadronic calorimeter. The calorimeters and tracking elements are inside a $4 \mathrm{~T}$ solenoidal magnetic field. The electromagnetic calorimeter is expected to achieve resolution of $\sigma(E) / \sqrt{E(G e V)} \sim 2.7(5.7) / E \oplus 0.55 \%$ in the central (forward) region. The hadronic calorimeter will have resolution of $\sigma(E) / \sqrt{E(G e V)} \sim 100 / E \oplus 5 \%$.

\subsection{Event Reconstruction and Pre-selection for $t \bar{t}$}

Although $t \bar{t}$ production is a rare process, it is at a sufficiently high scale to generate observable quantities that can be triggered online and selected offline with high efficiency. However, the ability to discriminate $t \bar{t}$ from background is another story and depends to a greater extent on details of particular final state topologies and detector capabilities. As discussed earlier, top quarks within the SM are predicted to decay into a $W$ boson and a $b$ quark with a branching fraction of nearly $100 \%$ [11]. The presence or absence of leptonic $W$ decays is then the primary factor in shaping final state signatures. One-third of $W$ bosons decay to leptons, including $\tau \nu_{\tau}$ with the $\tau$ decaying about one-third of the time to an $e$ or $\mu$ plus two neutrinos. The remaining two-thirds of $\tau$ decays involve hadrons and one neutrino. In view of these considerations, $t \bar{t}$ final states with 0,1 , or 2 potentially well-isolated electrons or muons from $W$ decays will occur at rates of roughly $54.9 \%, 38.4 \%$ and $6.7 \%$, respectively. These will be respectively referred to as the "all-hadronic", "lepton plus jets", and "dilepton" modes or channels of top events, where it is generally understood that "lepton" in this context refers to an $e$ or $\mu$. Whenever $\tau$ leptons are used, they are called out explicitly, as for example is done for the $\ell \tau$ dilepton channels. All $t \bar{t}$ final states include two high energy jets arising from fragmentation of $b$ quarks. The leptonic modes (including hadronic $\tau$ decays) have an imbalance in transverse momentum $\left(\mathbb{E}_{T}\right)$ due to the presence of undetected neutrinos. There are two additional jets for each hadronic $W$ decay and there are sometimes additional jets from QCD radiation. Existing collider experiments can identify isolated leptons from $W$ decays with very high efficiency and they can also measure their momenta with very good resolution. Jets and $\mathbb{E}_{T}$ in $t \bar{t}$ events are also easily detected but are not as well-resolved. It is worth noting that almost all high energy events at hadron colliders contain only jets because energetic isolated electrons and muons are due to rare weak decays involving real or virtual intermediate vector bosons. Lepton multiplicity is therefore highly correlated (anti-correlated) with signal purity (abundance) in event samples selected for $t \bar{t}$ studies.

Most measurements of top quark properties begin with the determination of the production cross section simply because it is almost a direct by-product of the selection of an event sample, including optimization of the discrimination of $t \bar{t}$ from backgrounds and establishing the detailed make-up of the final sample. Event sample selection can be divided into three stages; (i) online object (e.g. $e, \mu$, jet, $\mathbb{E}_{T}$ ) reconstruction and triggering, (ii) offline event reconstruction and pre-selection and (iii) the use of specialized information and techniques to better distinguish $t \bar{t}$ events from backgrounds and to understand as well as possible the contributions and characteristics of $t \bar{t}$ and various background processes that enter into the final sample.

This section provides an overview of object reconstruction, triggering, event selection and some of the methods used to discriminate signal from background in $t \bar{t}$ studies performed by the four major 
hadron colliders discussed sections 4.1 and 4.2. In the interest of space and pedagogy, no attempt is made to provide every detail of object and event reconstruction for all published $t \bar{t}$ studies. Rather the emphasis here is on the general ideas behind, and motives for, using some of the more important methods. Many more details can be found in the references cited in the text. A review of $t \bar{t}$ cross section measurements is then presented in section 5 .

The basic components of $t \bar{t}$ events that need to be identified and reconstructed are; (i) the primary interaction vertex, (ii) electrons, (iii) muons, (iv) jets and (v) $\mathbb{E}_{T}$. Additionally, for better discrimination of $t \bar{t}$ from backgrounds, special methods are used to tag jets resulting from $b$ quarks and discriminating variables are constructed based on event topology and the kinematic properties of the objects in the event. Discriminating variables are used either for simple cut-based selection algorithms or as inputs to likelihood functions and neural networks. For any given object, all of the hadron collider experiments typically employ similar reconstruction and identification techniques, though possibly in different order or with a different emphasis, depending upon the particular strengths of the apparatus.

The primary vertex for a given event is found by associating well-measured tracks to a common point of origin using, for instance, a Kalman filter [156]. Interaction vertices are found event-by-event and their locations for a large number of events can be used to define the beamline in the luminous region, defined as the region along the $\mathrm{z}$ axis where the probability for interactions is high. The probability of an interaction as a function of $\mathrm{z}$ in this region has an approximately Gaussian distribution with $\sigma \sim 30(\sim 5) \mathrm{cm}$ at each Tevatron (LHC) interaction region with peak at, or near to, the nominal geometric center of the detector. Typically there is more than one interaction per bunch crossing and more than one interaction vertex may be reconstructed. Provided that the multiplicity is not too high, it is generally true that only one of the interactions involves a hard-scatter with high constituent CM energy. The others are typically soft-scatters known collectively as "minimum bias" events. The choice of a primary vertex to associate with $t \bar{t}$ production can thus be done in several ways. One can choose the vertex with the highest $p_{T}$ sum of associated tracks. In leptonic modes, the primary vertex can be chosen to be the one to which the lepton is associated. In D0 a "minimum bias probability" [157] is computed for each reconstructed vertex based on the transverse momenta and total number of associated tracks. The primary vertex with the lowest minimum bias probability can then be selected as the hard-scatter vertex.

Unlike primary vertex reconstruction, lepton reconstruction involves not only the tracking system but also additional detector subsystems specifically designed for this purpose. Electron reconstruction in its most basic form is the simple matching of a localized deposit of energy in the electromagnetic (EM) calorimeter with a track reconstructed in the tracking system. The tracks are required to be above some $p_{T}$ threshold (typically no higher than $10-20 \mathrm{GeV} / \mathrm{c}$ ) and must pass quality cuts which are usually based on the number of hits found on tracking elements and the quality of the track fit as measured by the $\chi^{2}$ per degree of freedom $\left(\chi^{2} / N_{d o f}\right)$, for example. The energy deposited by an electron should be well-contained in the electromagnetic section which is designed and built with a large number of interaction lengths. The energy is also expected to have a relatively small lateral spread in $\eta-\phi$ in the absence of bremsstrahlung. The EM cluster is obtained by combining signals from neighboring cells using a variety of methods from simple seed-based, fixed proximity groupings or patterns, to algorithms using a small radius cone in $\eta-\phi$ such as $R=\sqrt{\Delta \eta^{2}+\Delta \phi^{2}} \sim 0.2$. More sophisticated algorithms that take into account bremsstrahlung photons by recapturing isolated EM clusters separated in azimuth from the main cluster are also employed in some cases. Electron discriminating variables include the relative proportions of hadronic and electromagnetic energy such as the ratio $E_{H A D} / E_{E M}$ or the EM fraction $\left(f_{E M}=E_{E M} / E\right)$ of the cluster energy. In D0 [157] a fixed cut, $f_{E M}>0.9$, is used while in $\mathrm{CDF}$ [158] it is energy dependent; $E_{H A D} / E_{E M} \leq 0.055+0.00045 E(G e V)$. More fine-grained detector elements in ATLAS [119], CDF and D0, provide additional shower shape information that is used to build likelihood discriminants.

It is interesting to note that the high radiation environment of the LHC has led to a greater use 
of silicon detectors for tracking as discussed in sections 4.1 and 4.2. This has resulted in an increase in the amount of material which leads to a substantial probability for bremsstrahlung radiation for low $E_{T}$ electrons. This has important implications for $e$ and $\gamma$ reconstruction. In CMS [159] for instance, the Kalman filter track algorithm valid for $p_{T} \geq 30 \mathrm{GeV}$ is replaced by a non-linear GaussianSum Filter (GSF) [160] in which state vectors and errors are Gaussian mixtures of components whose weights depend on the measurements. Bethe-Heitler [161 modeling of energy loss in tracking material is also performed. The track parameters that are obtained are more meaningful along the entire track trajectory which allows more powerful matching discriminants to be employed because the difference between the estimated $p_{T}$ of the electron at the primary vertex and that at the face of the EM calorimeter is a good measure of the fraction of energy lost to bremsstrahlung.

Muon reconstruction in its simplest form involves a match between a track in the tracking system and a track, segment or simply some number of hits in the outer muon stations. In CDF and D0 isolated high $p_{T}$ tracks in the tracker that pass standard quality cuts are extrapolated to the muon chambers situated beyond the calorimeter in search of a matched muon track segment. In D0 segments are defined when at least 2 (1) wire (scintillator) hits found in the three stations are matched. In ATLAS and CMS, the muon systems are used to obtain standalone muon reconstruction and $p_{T}$ measurements. Global muons in CMS correspond to muon tracks that are matched to tracker tracks. In all cases, the tracker provides most of the muon track parameter information. However, at very high momentum, the large lever arms of the CMS and ATLAS muon systems improve the $p_{T}$ measurements substantially. All experiments apply additional requirements to minimize contamination from a number of sources including hadron punch-through, decay in flight (DIF) hadrons, beam-halo and Cosmic ray muons. Punch-through hadrons are those rare cases in which hadrons from hadronic showers manage to escape the calorimeter and generate signals in the muon stations. Decay in flight hadrons involve real muons as in $\pi^{+} \rightarrow \mu \nu_{\mu}$ and $K^{+} \rightarrow \mu \nu_{\mu}$. These are dominant decay modes at nearly $100 \%$ and $63.5 \%$, respectively, but the lifetimes are long $\left(c \tau_{\pi}=7.8 \mathrm{~m}, c \tau_{K}=3.7 \mathrm{~m}\right)$ and they are generally highly boosted so that the decays occur relatively rarely inside the detectors. Also, when they occur they are generally inside jets and can be suppressed, but not entirely eliminated, by a variety of clean-up and isolation requirements. In the case of $t \bar{t}$ the candidate muons are from $W$ decays and so they are prompt (i.e. originating from the primary vertex) and generally well-isolated. Useful requirements are that the calorimeter energy should be consistent with what is expected for a minimum ionizing particle (MIP) such as a muon. The distance of closest approach $d$ to the primary vertex in the transverse plane divided by its uncertainty, also known as impact parameter significance $S_{d}$, should be consistent with what is expected for a prompt track (e.g. $S_{d} \equiv d / \sigma_{d} \leq 3$ in CDF). The track must also match to the appropriate primary vertex in z.

For both electrons and muons, there are different classes of quality that are defined, called "loose", "medium" and "tight" etc., for which progressively stronger requirements are used, and lower efficiencies are obtained. The key element in this progression is lepton isolation. Calorimeter and track isolation require that the fraction of summed $E_{T}$ of calorimeter towers or the summed $p_{T}$ of tracks in a cone of radius $R \sim 0.4-0.5$ about the centroid of the calorimeter cluster or the lepton flight path, excluding the contribution of the lepton itself, should be small, or a small fraction of the energy of the lepton. Requirements are usually on the order of a maximum of 1 to $6 \mathrm{GeV}$ or a fraction not to exceed $\sim 0.1$.

In all cases, lepton triggering and reconstruction efficiencies are studied via $Z \rightarrow \ell \ell$ decays. The basic goal is to obtain a large, pure and unbiased sample of high $p_{T}$ isolated leptons by using as few lepton discriminants as possible. The procedure, which dates back (at least) to the CERN SPS collider experiments is now known as "Tag and Probe" and typically begins with a sample of single lepton triggers that each contain a corresponding high $p_{T}$ tight lepton. The next step is to identify a potential second high $p_{T}$ lepton in the event using fewer lepton discriminants. If the two objects form an invariant mass that is comparable to the $Z$ mass, then it is very possible that the second candidate is a true lepton. In practice, the invariant mass distribution for many such events will show a peak at $M_{Z} \sim 91 \mathrm{GeV}$ over a background that can be measured in sidebands. The tight and loose selection criteria for the two 
lepton candidates can be varied to obtain an acceptable signal-to-background ratio. At this point, one has an unbiased sample of real leptons. These represent the denominator in subsequent measurements of efficiency. For instance one can select the second lepton without requiring it to have fired a lepton trigger and then see how often it has done so to extract a relative trigger efficiency. More generally, one can measure the relative efficiency of any criteria that was not used to select the probe lepton.

Like electrons, jets are constructed from calorimeter towers but are generally broader and involve substantial energy deposits in both the EM and the hadronic layers. They are frequently constructed from calorimeter cells and towers using iterative cone algorithms of various types. CDF and ATLAS use a default cone size of $R=0.4$ while CMS and D0 use $R=0.5$. In some cases there are multiple algorithms run all of whose results are available for use in an analysis. These may include infrared safe cone and $K_{t}$ algorithms [162. Once candidate jets are formed, selections may be applied to avoid problems associated with noisy towers and to remove electrons and photons. The EM fraction, for instance is required to be neither too small nor too large (e.g. $0.05 \leq f_{E M} \leq 0.95$ ). In addition there can be requirements on the longitudinal and lateral energy distributions. D0 for instance requires that the ratio of leading to next-to-leading $E_{T}$ cells be less than 10 , that no tower contain more than $90 \%$ of the total jet energy and that the fraction of energy in the outermost hadronic layer be less than $40 \%$. In all experiments, towers or cells associated with an identified $e$ or $\gamma$ are removed and one applies corrections to take into account response imperfections and other effects. The latter may include a correction for the energy offset due to contributions from the underlying event, pile-up of signal from successive bunch crossings or multiple interactions within a single bunch crossing, and noise in the calorimeter electronics. There is an additional correction for the $\eta$ dependence of calorimeter response and possibly for time dependence of the response. The latter is particularly important in calorimeters that use scintillators as active elements since the light yield diminishes with age and radiation dose. The jet energy scale (JES) is obtained from precisely measured EM objects by assuming $E_{T}$ conservation in $\gamma+$ jet events in order to obtain an average jet correction as a function of $E_{T}$. At $E_{T} \approx 10 \mathrm{GeV}$ jet corrections can be as large as $100 \%$ but are generally no more than $\sim 5-10 \%$ at $E_{T} \geq 100$.

The presence of a $\nu$ is inferred from energy imbalance in the transverse plane, or $\mathbb{E}_{T}$. The $x$ and $y$ components of raw $\mathbb{E}_{T}$ are calculated simply as the negative sum over the corresponding components of the $E_{T}$ in calorimeter cells. The sum includes all calorimeter cells that remain after noise suppression algorithms and corrections are applied. Several corrections are then made for cells associated with objects for which energy corrections are known. Thus the jet energy corrections that are applied to jets are subtracted vectorially. Similarly, the generally smaller energy corrections applied to clusters associated with electrons and photons are vectorially subtracted and the small vector $E_{T}$ contribution from a muon in the calorimeter is added while the muon $p_{T}$ vector is subtracted. Finally, additional corrections are applied for $E_{T}$ losses due to gaps in coverage and dead material such as occurs in the walls of LAr cryostats.

Triggering and preliminary event selection of leptonic modes are based upon the presence of at least one high $E_{T}$, isolated $e$ or $\mu$ and/or large $\mathbb{E}_{T}$ as defined in the preceeding section. To avoid various backgrounds, as discussed below, thresholds for these quantities are set as high as possible without incurring substantial loss of $t \bar{t}$ signal. It turns out that the optimal threshold for leptons in $t \bar{t}$ events is roughly the same at the LHC as at the Tevatron. In fact, the threshold is typically around $20 \mathrm{GeV}$ which is the level where one would successfully retain most leptons coming from $W$ 's produced at rest. This is the result of several factors. First, top quarks are produced with very little excess momentum because they are produced near threshold as a result of steeply falling parton distributions for $q \bar{q}$ annihilation at the Tevatron and $g g$ fusion at the LHC. Secondly the boost imparted to the $W$ from the decay of the top quark has little effect on the position of the peak in the lepton energy distribution for the decay $W \rightarrow \ell \nu$. This is because of the polarization of the $W ; \sim 70 \%$ longitudinal and $\sim 30 \%$ left-handed polarization, which means that the $\ell$ is on average emitted in a direction slightly opposed to the direction of flight of the $W$, neatly canceling out the effect of the boost. The most probable value 

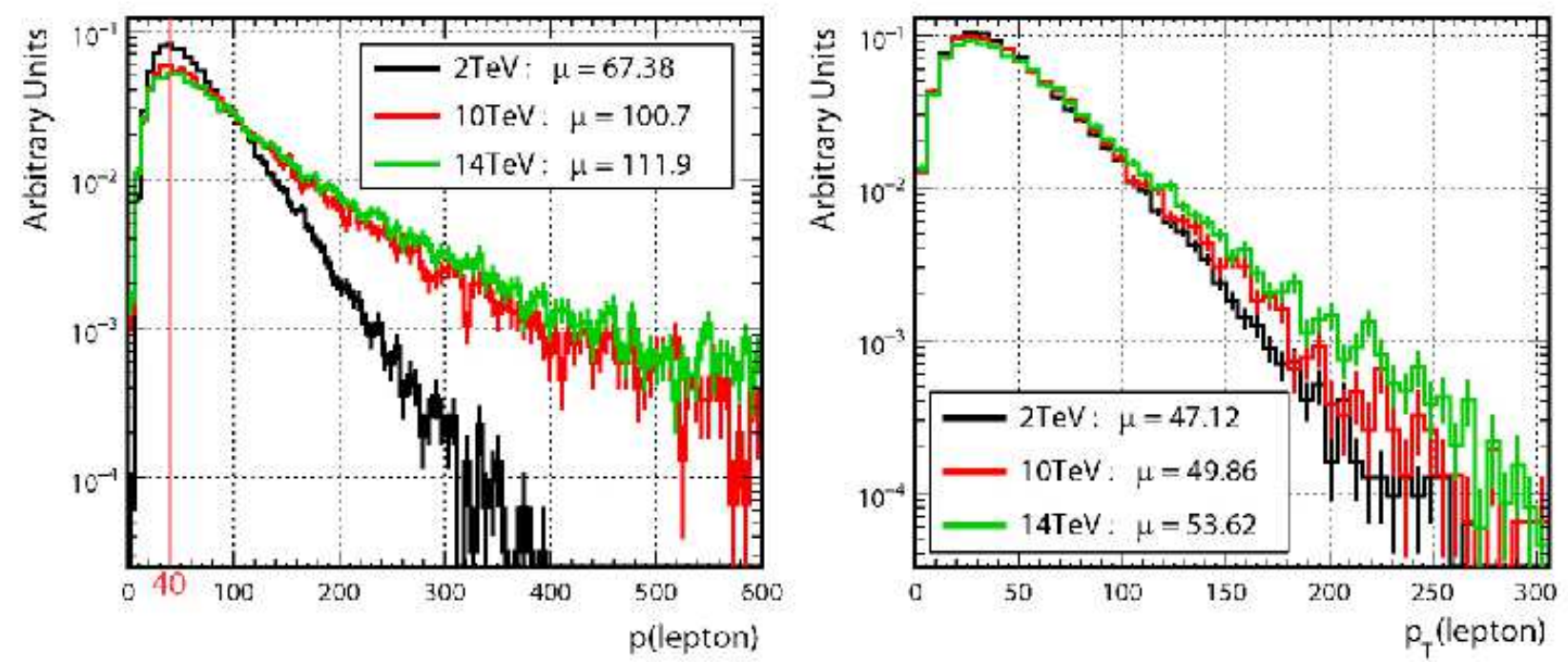

Figure 9: Lepton $p$ (left) and $p_{T}$ (right) for $e$ 's and $\mu$ 's from $W$ decays in $t \bar{t}$ at the Tevatron and LHC.

of the energy of the $\ell$ stays quite near to $M_{W} / 2 \sim 40 \mathrm{GeV}$ as seen in the generator level distributions shown in Figure 9. A cut on $p_{T}>20 \mathrm{GeV}$ thus retains the peak of the distribution of the leptons over the entire central region of the detector, $(|\eta| \leq 1.3)$. Of course a threshold of $20 \mathrm{GeV}$ is less efficient for an $e$ or $\mu$ coming from the decay chain $W \rightarrow \tau \nu$, with $\tau \rightarrow \ell \nu_{\ell} \bar{\nu}_{\tau}$ due to the energy carried by the two additional neutrinos.

The $t \bar{t}$ all-hadronic mode is selected by requiring multijet final states. For triggering purposes the calorimeter granularity can be simplified as in the case of CDF for which it is a $24 \times 24$ grid of "trigger towers" in $\eta-\phi$ with each tower spanning $\Delta \phi \times \Delta \eta=15^{\circ} \times 0.2$. In the most recent study of all-hadronic $t \bar{t}$ events by CDF [163] with $1.02 \mathrm{fb}^{-1}$ of data, the triggering at each level proceeds as follows. Level 1 requires a single tower with $E_{T} \geq 10 \mathrm{GeV}$. Level 2 requires $\sum_{j} E_{T, j} \geq 175 \mathrm{GeV}$ where the sum is over all trigger towers. Using more accurate energy estimates, Level 3 requires at least four jets with $E_{T} \geq 10 \mathrm{GeV}$. It is in fact relatively typical to find that the trigger and event pre-selection require at least four jets above an $E_{T}$ threshold of order 10-30 GeV. This is a particularly difficult channel because of the extremely large multijet backgrounds. After the final stage of triggering the CDF event sample contains 4.3 M events with a Monte Carlo (MC) estimated efficiency of $80 \%$ for all-hadronic $t \bar{t}$ events, corresponding to a $S / B$ of roughly $1 / 1100$ where the background is essentially all multijet events. Further improvements in event selection for all $t \bar{t}$ modes and the all-hadronic mode in particular requires more discriminating power.

It is very important to note that in the preceeding discussion the triggers and event selections do not make use of all of the components of the corresponding $t \bar{t}$ signatures. In particular there are always two additional b jets and two additional light quark or charm jets per hadronic $W$ decay. Indeed, for 0 , 1 and 2 lepton modes, the main concentrations of signal appear in samples of events with $\geq 2, \geq 4$ or $\geq 6$ jets, respectively. Similarly, the purity of the selected samples increases with each additional $b$ jet that's identified. The reason that these event characteristics are not used in the standard event selection (often called "pre-selection") is that it is important to have access to events from signal-depleted regions which are similar in many respects to events in signal regions. These provide access to control samples that can be used to test one's understanding of important SM backgrounds via data-driven and/or MC-based methods. The ability to extrapolate an understanding of backgrounds in control regions to 
the signal region can range from very direct and simple to very tenuous and speculative. In general, it is somewhere between these extremes. It is however one of the most important stages of the analysis for the experimentalist because one cannot generally rely upon simulations to provide an accurate characterization of the behavior of many backgrounds or of the signal. Many of the more important and creative contributions to experimental hadron collider physics and $t \bar{t}$ studies in particular have been associated with this stage of analysis.

Once the events have been triggered online and pre-selected offline, the next step is generally focused on methods for enhancing the $S / B$ ratio while retaining a substantial signal component. Ideally the methods employed do not bias the kinematic properties that are likely to be studied after a cross section is measured. A good example is the use of $b$ tagging. The most powerful techniques for identification of $b$ jets, or " $b$ tagging" are based upon the fact that $b$ hadrons have long lifetimes $(\tau \sim 1.5$ ps $)$ so that with typical transverse energies of 50 to $100 \mathrm{GeV}$ in $t \bar{t}$ events they have a mean travel path of order $5 \mathrm{~mm}$. If the $b$ hadron decays to two or more charged stable particles, it can often be detected via a well-reconstructed vertex that is significantly displaced from the primary vertex [46, 164, 151]. Since a $b$ hadron generally decays sequentially to a $c$ meson which also has a fairly long lifetime, it is possible for the visible charged tracks to originate from two different displaced vertices. In some cases, this spoils the ability to isolate a well-reconstructed vertex. Nevertheless, the $b$ jet can be tagged by algorithms that rely upon the low probability for jets of lighter flavor partons to produce tracks with large impact parameters $(d)$ relative to the primary vertex [165, 158] or beamline. Alternatively a $b$ tag can rely upon the appearance of an $e$ or $\mu$ that is closely associated with a hadronic jet [166, 167]. In the latter case, the lepton is assumed to result from the semileptonic decay of the $b$ or subsequent $c$ meson. Such leptons are more difficult to identify than those coming from $W$ decays because they are not isolated. However, they do retain many of the characteristics of leptons and often have a substantial component of their momentum transverse to the jet axis. They also often have large positive impact parameters but this information is not always used in order to retain high efficiency and avoid correlation with the lifetime tagging techniques.

Secondary vertex tagging in CDF operates on a per-jet basis, where only tracks within the jet cone are considered for each jet in the event. Tracks are selected based on $p_{T}$, track fit quality $\left(\chi^{2} / N_{d o f}\right)$ and the quality of associated Silicon detector hits because only tracks whose impact parameters are well-resolved will contribute usefully to secondary vertex reconstruction. Jets with at least two such tracks can produce a displaced vertex and so are called "taggable". The CDF SecVtx algorithm [168] developed in Run 1 uses a two-pass approach to find secondary vertices. In the first pass, relaxed track requirements are used $\left(p_{T}>0.5 \mathrm{GeV} / \mathrm{c} S_{d}>2.5\right)$, but high purity is achieved by requiring at least three tracks of which one has $p_{T}>1.0 \mathrm{GeV} / \mathrm{c}$. If the first pass is unsuccessful, track requirements are tightened $\left(p_{T}>1.0 \mathrm{GeV} / \mathrm{c} S_{d}>3\right)$ and an attempt is made to find a vertex with two tracks of which one has $p_{T}>1.5 \mathrm{GeV} / \mathrm{c}$. Once a vertex is found, the two dimensional (2D) decay length $\left(L_{2 D}\right)$ is calculated as the projection of the distance from the primary to the secondary vertex projected onto the jet axis in the transverse plane. The sign of $L_{2 D}$ is positive (negative) if the angle between the jet axis and the vector from the primary to secondary vertex in the transverse plane is less (more) than $90^{\circ}$. The latter corresponds to the unphysical situation in which the secondary vertex is behind the primary vertex relative to the jet direction. Secondary vertices corresponding to the decays of $b$ and $c$ hadrons generally have large positive values of $L_{2 D}$. Those from mismeasured tracks ("mistags") will have smaller, possibly negative values. The latter are suppressed by requiring "positive tags" with $S_{L_{2 D}} \equiv L_{2 D} / \sigma_{L_{2 D}}>3$. Nevertheless, "negative tags" with $S_{L_{2 D}}<-3$ are used to estimate the false positive tag rate. Note that values of $\sigma_{L_{2 D}} \sim 100-200 \mu \mathrm{m}$ are typical. Other experiments use similar but different algorithms based on secondary vertex finding. In D0 for instance [151], jets are formed as clusters of tracks and secondary vertices prior to being associated to calorimeter jets. As for CDF, track selection relies upon the quality of Silicon detector information, and impact parameter significance and a calorimeter jet is $b$ tagged if it has significant positive $2 \mathrm{D}$ decay length. In all instances, care is 
taken to reject tags consisting of pairs of tracks that construct an invariant mass comparable to known long-lived light flavor mesons such as $K_{S}^{o} \rightarrow \pi^{+} \pi^{-}$and $\Lambda^{o} \rightarrow p^{+} \pi^{-}$or photon conversions $\gamma \rightarrow e^{+} e^{-}$.

In the absence of vertexing, there are several commonly used algorithms for $b$ tagging. The simplest is the "track counting" method in which one counts tracks associated with a particular jet that have positive impact parameter significance above some threshold and declare a candidate $b$ tag when there are some minimum number of these tracks. In some cases [169] a jet is $b$-tagged if there are $N \geq 2(\geq 3)$ tracks with $S_{d}>3(>2)$. The $b$-tag efficiency in this case is slightly higher than that for secondary vertexing but a drawback is that the mistag rate is doubled. Another drawback of this algorithm is that it does not take into account the fact that the probability for a jet of light flavor origin $(u d s$ or $g)$ to pass the tagging requirements increases with the $E_{T}$ of the jet as a result of increasing numbers of tracks inside the jet cone. This means that the mistag rate rises with $E_{T}$. The problem is overcome in a more sophisticated version of the track counting algorithm known as the "jet probability" algorithm [165, 158]. For this algorithm, the negative side of the track impact parameter distribution is mirrored about zero to obtain a data-driven definition of positive impact parameter resolution. This is used to determine a per-track probability that a track with a given significance $S_{d}$ is in fact consistent with having come from the primary vertex. The products of such probabilities for all tracks in the jet is then used to define a jet probability $P_{J}$ which is the probability that an ensemble of $N$ tracks in the jet cone with impact parameter significances $S_{d}^{j}$ for $j=1, \ldots, N$ could be produced by fragmentation and hadronization of a light flavor parton with no lifetime. The negative impact parameter distributions are obtained from large samples of jet events for which the contamination of heavy flavor is small. To take into account possible variations in track parameter resolutions, CDF defines 72 different track categories corresponding to different values of $\vec{p}_{T}, \eta$ and the number of silicon detector hits on the track. Resolution functions for each category are a convolution of four Gaussian distributions. The jet probabilities constructed from the per-track probabilities are properly normalized to take into account the number of tracks contributing to the calculation of $P_{J}$. As a result, a sample of jets originating from light flavor partons will have a $P_{J}$ distribution which is flat from 0 to 1 while the distribution for a sample of jets originating from heavy flavor $(b$ and $c$ ) partons will tend to be concentrated at very low probabilities. The algorithm has a stable mistag rate as a function of jet $E_{T}$. It also has the attractive feature of providing a continuous variable, the value of $P_{J}$, for use in selecting events.

The algorithms described above have the potential for efficient identification of $b$ jets with fairly low mis-identification rates. Purity can be increased at the cost of efficiency, but it is difficult to extend their performance beyond efficiencies of order 50-60\%, and so more sophisticated approaches are taken for additional gains. D0 for example uses a neural-network $b$ tagger [170] and the LHC experiments have developed a variety of tagging algorithms including neural networks and methods that combine vertex, impact parameter and jet shape information into multivariate discriminants [159].

Measurements of $t \bar{t}$ production cross sections that rely upon $b$-tagging assume the SM branching ratio $\operatorname{Br}(t \rightarrow W b) \sim 100 \%$. There are however physics models in which this is not true and so an interesting approach to $t \bar{t}$ signal discrimination is the use of event topology information. D0 has used a set of 13 variables to build a discriminant function [157] in the lepton plus jets channel. Compared to $t \bar{t}$ events in which the final state objects are distributed somewhat isotropically, background events from hard-scatter processes have more of a "back-to-back" structure. These observations can be quantified using aplanarity $\mathcal{A}=\frac{3}{2} \lambda_{3}$ and sphericity $\mathcal{S}=\frac{3}{2}\left(\lambda_{2}+\lambda_{3}\right)$, defined by the normalized 2D momentum tensor $\mathcal{M}_{i j}=\sum p_{i} p_{j} /\left|\vec{p}^{2}\right|$ of the jets in the event and the $\lambda_{k}$ are ordered eigenvalues of the $\mathcal{M}_{i j}$ (with $\lambda_{1}$ the largest). In top quark events, these quantities are larger than for background. In addition, $t \bar{t}$ events are produced close to threshold and so have less longitudinal boost, resulting in a larger proportion of energy in the central rapidities. One thus obtains discrimination power from centrality $\mathcal{C}$, defined as the ratio of the scalar sum of $p_{T}$ of the jets to the scalar sum of the energy of the jets. Other useful variables include $H_{T} \equiv \sum p_{T}$ and $M_{T}$ which are the scalar sum $p_{T}$ and transverse mass, respectively, of the four leading jets in the event. 


\section{$5 \quad$ Measurement of the $t \bar{t}$ Production Cross Section}

Measurements of the top pair production cross section $\left(\sigma_{t \bar{t}}\right)$ can be sensitive to new particles which come from or decay to top quarks. Processes that could enhance $\sigma_{t \bar{t}}$ include Little Higgs [171], and strong dynamics [172]. Supersymmetry [173] could produce multi-lepton, multijet signatures with significant $\mathbb{E}_{T}$ akin to $t \bar{t}$ signatures and possibly also the cascade $t \rightarrow H^{+} b \rightarrow \tau \nu b$ which would alter observed top quark branching ratios. Conversely $t \bar{t}$ production must be well understood at the LHC where it is a background for most new physics models. Of course, $\sigma_{t \bar{t}}$ is also of interest in its own right along with other properties of this conspicuously heavy quark. In Run 2, CDF and D0 have accumulated $\sim 50$ times the integrated luminosity of Run 1. Current theoretical uncertainties on top production at the Tevatron have $\sim 10 \%$ uncertainties. At the LHC the product of cross-section and luminosity will be roughly three orders of magnitude higher, making the LHC a true top quark factory.

The cross section is determined by the number of observed candidate events $N_{o b s}$, the estimated number of background events $B$, the integrated luminosity $\int \mathcal{L} d t$, and the $t \bar{t}$ acceptance times efficiency $\mathcal{A} \times \epsilon$. The latter is defined as the fraction of simulated $t \bar{t}$ events that pass all selection criteria after correcting for known differences between real and simulated data as estimated with real and simulated control samples. With regard to the latter, there are a variety of programs regularly used. Exact leading-order calculations such as ALPGEN [174] and MADGRAPH [175] provide the partonic final states underlying some important background processes such as $W / Z+n$ jets with $n=1,2,3, \geq 4$. These programs are coupled with showering MC such as PythiA [176] and Herwig [177] which are often used for $t \bar{t}$ signal samples and rare electroweak backgrounds such as di-bosons. Heavy flavor decays are handled by programs such as EvTGEN [178]. In all cases, the GEANT [179, 180] program is used to simulate the response of the detectors. More details on Monte Carlo routines used in specific measurements of the $t \bar{t}$ production cross sections are available in the cited references.

The final cross section can be calculated by maximizing the likelihood of obtaining $N_{\text {obs }}$ events given the number expected $N_{\text {exp }}=S+B$, where $S=\sigma_{t \bar{t}} \mathcal{A} \epsilon \int \mathcal{L} d t$. Uncertainties are then taken from the cross section values where the logarithm of the likelihood decreases by 0.5 , and systematic uncertainties are included in the likelihood as nuisance parameters with Gaussian probability distributions. The result of the likelihood maximization is equal to that obtained from the familiar formula $\sigma_{t \bar{t}}=\left(N_{o b s}-\right.$ $B) /\left(\mathcal{A} \epsilon \int \mathcal{L} d t\right)$, and yields statistical uncertainties for a Poisson probability distribution while also having the capacity to extract a single cross section from multiple data samples.

What follows is a broad-brushed presentation, emphasizing important methods and results. Details are available in the references. Unless otherwise specified, the cross section values presented in this section assume $M_{t}=175 \mathrm{GeV} / \mathrm{c}^{2}$.

\subsection{Measurements of $\sigma_{t \bar{t}}$ in the Dilepton Channel}

At hadron colliders the main SM processes with true high- $p_{T} e \mu$ dilepton final states are $t \bar{t}, W^{ \pm} W^{\mp}$ and $Z / \gamma^{*} \rightarrow \tau \tau \rightarrow e \mu \nu_{e} \nu_{\mu} \nu_{\tau} \nu_{\tau}$. These processes are distinguishable from one another by jet counts and $\mathbb{E}_{T}$. There is typically less $\mathbb{E}_{T}$ in $Z \rightarrow \tau \tau$ events due to the many neutrinos which tend to wash out their collective impact and steal energy from the other leptons $(\ell=e, \mu)$. The $W / Z$ processes are generally produced with fewer and softer jets than $t \bar{t}$. The hadronic tau decay channels, labeled as $\ell \tau$ and $\tau \tau$, arise from these same processes but their experimental signatures are not as distinctive. Nevertheless, they can be statistically separated from jets to some extent, as discussed below. The ee and $\mu \mu$ final states again involve the same SM processes but must also contend with Drell-Yan (DY) $Z / \gamma^{*} \rightarrow \ell^{+} \ell^{-}$. To combat this, one raises the $\mathbb{E}_{T}$ threshold for all opposite-sign same-flavor (OSSF) dileptons and either vetoes events in the "Z mass window", $\left(\left|M_{\ell \ell}-M_{Z}\right| \leq 15 \mathrm{GeV}\right)$, or raises the $\mathbb{E}_{T}$ threshold further there.

In addition to sources of real lepton pairs, there are "instrumental backgrounds" in which leptons are 
faked by jets or other types of leptons. An isolated $\mu$ could be faked by a jet that fragments primarily to a single leading $p_{T}$ charged hadron that decays to a $\mu$, or by a hadron that punches through the hadron calorimeter to leave a signal in the muon system. A jet containing mainly $\pi^{o}$ 's can fake an isolated $e$ if a high $p_{T}$ electron from $\gamma \rightarrow e e$ provides a matched track. Hadronic $\tau$ decays have signatures similar to very narrow jets with low multiplicities of charged hadrons. Thus for $e e, e \mu, \mu \mu$, and $\ell \tau$ samples a significant background is $W+$ jets with $W \rightarrow e \nu, \mu \nu$ or $\tau \nu$ and a fake $e$ or $\mu$. A smaller background arises from multijet events requiring two fake leptons and fake $\mathbb{E}_{T}$. Though rare, these "QCD" events do occur because the QCD production cross section is many orders of magnitude higher than those for processes with real high $p_{T}$ isolated leptons.

Of course $t \bar{t}$ events always contain a pair of bottom quarks while the other SM sources of real or fake dileptons rarely do. Thus $b$ tagging can be used to improve $S / B$ in dilepton events. This was rarely done in earlier Tevatron measurements because of a shortage of events and the inherent purity of the $t \bar{t}$ dilepton signature. Larger dilepton samples are now available and analyses include $b$ tagging to produce ultra-pure $t \bar{t}$ samples.

The most recent CDF measurement of $\sigma_{t \bar{t}}$ in the dilepton channel [181] makes use of $2.8 \mathrm{fb}^{-1}$ of data. The basic selection and treatment of backgrounds is typical of most such measurements. Event selection includes two opposite sign (OS) leptons, large $\mathbb{E}_{T}$ and two jets with $E_{T}>30$ and $E_{T}>15 \mathrm{GeV}$. Remaining backgrounds fall into two categories as discussed above in which both leptons are real or at least one is fake. Diboson $(W W, W Z, Z Z)$ and $Z / \gamma^{*}$ are estimated from MC samples taking into account acceptances, efficiencies, theoretical cross sections, and the appropriate integrated luminosity. Differences between simulation and data are corrected as discussed in section 4.3. Jet multiplicity scale factors for processes involving multiple jets from QCD radiation such as $W W$ and $Z / \gamma^{*}$ are also implemented. The contribution of events with a fake lepton is estimated with a sample of same sign (SS) dileptons having the same kinematic selection as the OS sample. It is assumed that for the $W+$ jets background a fake lepton will pair with a real lepton with the same or opposite sign at the same rate. This is verified in the background dominated $e \mu+\leq 1$ jet control sample. Kinematic and geometric acceptance $\mathcal{A}$ is determined in simulation. Prior to $b$ tagging, the "pre-tag" sample has a total of 162 events and an estimated background of $51.9 \pm 4.5$ events. The dominant uncertainties are in the estimated background from fake leptons and the jet energy scale (JES). Requiring at least one $b$ tag leaves 80 events with a background $4.0 \pm 1.7$ events. The cross sections obtained without and with a $b$-tag requirement are: $\sigma_{t \bar{t}}^{0 b}=6.67 \pm 0.77$ (stat) \pm 0.43 (syst) \pm 0.39 (lumi) pb, and $\sigma_{t \bar{t}}^{1 b}=7.81 \pm 0.92$ (stat) \pm 0.68 (syst) \pm 0.45 (lumi) pb where the systematic uncertainty is a convolution of acceptance and background uncertainties. The $\mathbb{E}_{T}$ distribution for events in the pre-tag sample and the lepton $E_{T}$ distribution in the tagged sample are shown in Fig. 10,

The first CDF dilepton measurement in Run 2 [182] did not use $b$ tagging but combined two measurements, one of which required the standard OS leptons while the other required only one $e$ or $\mu$ paired with an OS, isolated high $p_{T}$ track (referred to as a "track-lepton"). For a data sample of $197 \pm 12 \mathrm{pb}^{-1}$ they reported a cross section of $7.0_{-3.4}^{+4.4} \mathrm{pb}$. The track-lepton approach has the potential to increase the signal acceptance, particularly for $W \rightarrow \tau \nu$ decays, and so improves sensitivity to $t \rightarrow H^{+} b \rightarrow \tau \nu b$. The track-lepton approach has been refined and used with $1.1 \mathrm{fb}^{-1}$ by CDF [183] and $0.4 \mathrm{fb}^{-1}$ by D0 [184]. Note that the probability for a jet to fake a track-lepton is more than an order of magnitude higher than that for a jet to fake an $e$ or $\mu$, making the $W+$ jets background more significant. Indeed, with higher statistics samples the standard way of using jet data to parameterize the per-jet fake rates versus $E_{T}$ and $\eta$ begins to show inadequacies when applied to control samples like the $W+1 j$ sample. The CDF analysis used jets in $\gamma+$ jets and $Z+j e t s$ data to parameterize the per-jet fake rates applied to $W+$ jets events. The method takes into account the fact that quark jets have roughly an order of magnitude higher probability to fake a track-lepton than do gluon jets, and $W / Z / \gamma+$ jets events often contain a leading quark jet in the final state whereas dijets more often contain gluons. The new approach reduces the relative uncertainty on the fake lepton background to 
Pretag Top Candidates With Njet $\geq 2$

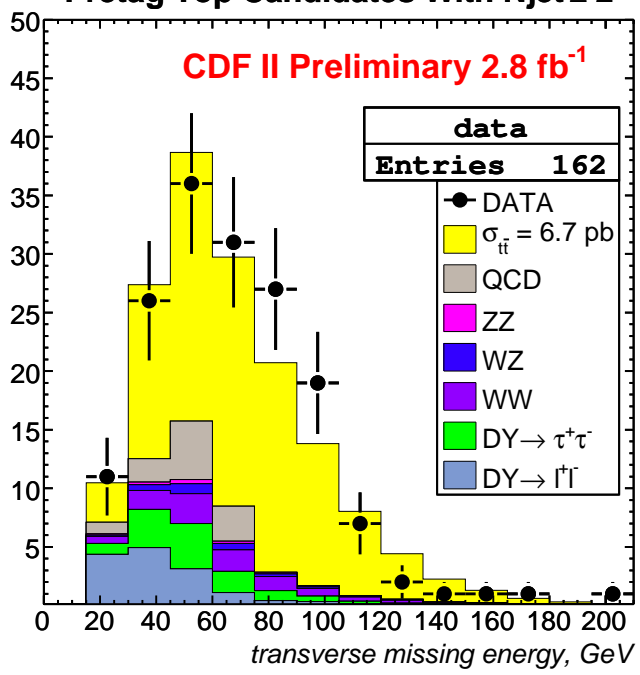

Tagged Top Candidates With Njet $\geq 2$

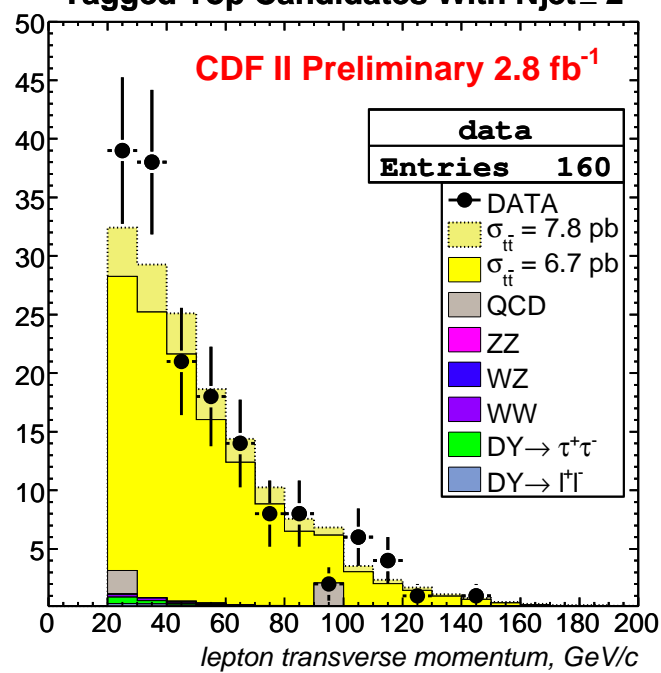

Figure 10: Comparison of data to expectations for missing $E_{T}$ for pre-tag (left) and lepton $E_{T}$ for tagged (right) CDF dilepton events [181].

$<20 \%$ from $30 \%-50 \%$ in prior Run 2 studies. Other sources of backgrounds for this analysis are $Z / \gamma^{*} \rightarrow e e, \mu \mu, \tau \tau$ in association with jets. The $\tau \tau$ contribution is estimated with MC events for which jet multiplicity is weighted to match data (e.g. $\ell \ell+j e t s$ events in the $Z$ mass window). The $e e$ and $\mu \mu$ contributions outside the $Z$ mass window that pass $\mathbb{E}_{T}$ cuts are estimated by multiplying the ratio of simulated DY events passing cuts outside and inside the $Z$ window times the observed number of events inside the $Z$ window. The final cross section combining tagged and untagged sub-samples is $\sigma_{t \bar{t}}=9.6 \pm 1.2$ (stat) ${ }_{-0.5}^{+0.6}$ (syst) \pm 0.6 (lumi) pb.

In the D0 analysis [184 the track-lepton category requires a $b$-tag. To extract the background with fake isolated muons D0 employs a "tight" sample containing $N_{T}$ events passing all $\mu \mu$ selection criteria and a "loose" sample of $N_{L}$ events with only one $\mu$ required to be isolated. The loose and tight event counts are then related to the signal-like events $\left(N_{s l}\right)$ and background-like events $\left(N_{b l}\right)$ in the "loose" sample where signal-like events contain muons from $W / Z$ decays and background-like events contain at least one muon candidate from sources associated with a jet. Thus $N_{L}=N_{s l}+N_{b l}$ and $N_{T}=\epsilon N_{s l}+f_{\mu} N_{b l}$ where $\epsilon\left(f_{\mu}\right)$ is the probability for the second muon in signal-like (backgroundlike) events to pass the isolation requirement. The same method expanded to four equations and four unknowns corresponding to pairings of real or fake leptons or track-leptons is used to estimate $W+j e t s$ and multijet backgrounds from special control samples. The efficiencies for the signal-like loose events to pass tight isolation criteria are obtained from simulated DY events while the corresponding efficiencies for background-like loose events were measured in multijet data with very low $\mathbb{E}_{T}$. The cross section obtained by combining all channels is $\sigma_{t \bar{t}}=7.4 \pm 1.4$ (stat) \pm 0.9 (syst) \pm 0.5 (lumi) pb.

More recently D0 carried out a study with a dataset of $1 \mathrm{fb}^{-1}$ [185] using a more sophisticated approach to $\tau$ identification. Three types of $\tau$ decays are defined and neural networks $\left(N N_{\tau}\right)$ for each type are developed using discriminating variables based on longitudinal and transverse shower profiles and calorimeter isolation. The three types of $\tau$ decay are characterized by a single track with energy deposited in the hadronic calorimeter (" $\pi^{ \pm}$-like"), a single track with energy in both the EM and hadronic calorimeters (" $\rho^{ \pm}$-like") and 2 or 3 tracks with invariant mass below 1.1 or $1.7 \mathrm{GeV}$, respectively. The cross section obtained for the combination of all channels is $\sigma_{t \bar{t}}=7.5 \pm 1.0$ (stat) ${ }_{-0.6}^{+0.7}$ (syst) ${ }_{-0.5}^{+0.6}$ (lumi) pb. The $\ell \tau$ channel was used to obtain a first measurement of the cross-section times branching ratio: $\sigma_{t \bar{t}} \times \operatorname{Br}(t \bar{t} \rightarrow \ell \tau b \bar{b})=0.13_{-0.08}^{+0.09}$ (stat) ${ }_{-0.06}^{+0.06}$ (syst) ${ }_{-0.02}^{+0.02}$ (lumi) pb for $M_{t}=170 \mathrm{GeV}$, in agreement 
with the SM expectation of $0.14 \pm 0.02 \mathrm{pb}$. In the $\ell \tau$ channel, QCD multijet backgrounds are partially reduced by requiring $15<\mathbb{E}_{T}<200 \mathrm{GeV}$ and a significant azimuthal separation between the $p_{T}$ of the $\ell$ and $\mathbb{E}_{T}$. The final cleanup of the $\ell \tau$ channel is provided by the requirement of at least one $b$-tag. The simulated background from $W+\geq 2$ jet events is normalized by fitting the transverse mass distribution of the isolated lepton and $\mathbb{E}_{T}$ to data. The multijet background is estimated with $S S$ events, taking into account $S S$ contributions from other backgrounds and $t \bar{t}$ with MC.

A final CDF dilepton measurement of note in recent years is one that employs a global fitting method [186] to simultaneously extract the production cross sections for $t \bar{t}, W^{+} W^{-}$and $Z \rightarrow \tau \tau$ events. These processes populate regions of the $\mathbb{E}_{T}-N_{j}$ plane to different degrees, where $N_{j}$ is the number of jets in the event. A study of the broader $\mathbb{E}_{T}-N_{j}$ plane has the potential to improve our understanding of all three processes and at the same time provide greater sensitivity to the appearance of discrepancies with the SM. The results for all three processes are consistent with SM expectations and the $t \bar{t}$ result is $\sigma_{t \bar{t}}=8.5_{-2.2}^{+2.7} \mathrm{pb}$.

\subsection{Measurements of $\sigma_{t \bar{t}}$ in the Lepton+Jets Channel}

The $\ell+j e t s$ channel $(\ell=e, \mu)$ is not intrinsically as pure as the dilepton channel. On the other hand, it has a much higher branching fraction and so can afford more stringent selection criteria to yield large event samples with good $S / B$. This is why the $\ell+j e t s$ channel played the largest role in the discovery of the top quark [46, 47] and carries the largest weight in the measurement of $M_{t}$ and many other top quark properties. The two main ways to obtain pure samples of $t \bar{t}$ events in this channel are the use of $b$ tags and event topology information. The main backgrounds are $W+$ jets events with $W \rightarrow \ell \nu_{\ell}$ and multijet events where a jet is misidentified as a lepton (as discussed in section 5.1) and energy is mis-measured resulting in substantial $\mathbb{E}_{T}$. The $t \bar{t}$ signal tends to occur with jet multiplicities $N_{j} \geq 4$ but there is also a non-negligible fraction with $N_{j}=3$. By contrast, the main backgrounds decrease rapidly with increasing jet multiplicities.

In the kinematic likelihood analyses of Ref. [157], D0 selects events with $N_{j} \geq 4$. The multijet background contribution is determined with data samples selected with loose and tight lepton identification as was discussed for the dilepton mode. The "loose - tight" sample, containing those events passing the loose, but not the tight criteria is used to model distributions of the variables used in the kinematic likelihood for multijets after correcting for residual $t \bar{t}$ and $W / Z+j e t s$ events. The variables, discussed in Section 4.3, exploit the topological differences between $t \bar{t}$ and backgrounds. They include aplanarity $\mathcal{A}$ and centrality $\mathcal{C}$, the $\sum p_{T}^{2}$ and $\sum \eta^{2}$ for the four leading jets, and many others. Templates for the distributions of these variables in $t \bar{t}$ and $W / Z+$ jets are based on simulated MC samples. The validity of the templates is tested on the $N_{j}=3$ sample. The templates are used in a maximum likelihood fit to the data. The multijet normalization is constrained to its estimated contribution within uncertainties. The $t \bar{t}$ cross section is measured separately for the different lepton types and all types combined. The combination result for $425 \mathrm{pb}^{-1}$ is: $\sigma_{t \bar{t}}=6.4_{-1.2}^{+1.3}$ (stat) \pm 0.7 (syst) \pm 0.4 (lumi) pb. D0 has recently published a result based on $0.9 \mathrm{fb}^{-1}$ [102] which alters somewhat the choice of discriminating variables that are used to include measures of the separation in $\phi$ and $\eta-\phi$ between pairs of objects. The analysis also includes the $N_{j}=3$ sample in the measurement, requiring that $\sum_{i=1}^{3} p_{T}{ }^{i}>120 \mathrm{GeV}$ for these events. They obtain $\sigma_{t \bar{t}}=6.62 \pm 0.78$ (stat) \pm 0.36 (syst) \pm 0.40 (lumi) pb. This is combined with an analysis in which at least one $b$ tag is required in place of the kinematic likelihood. The $b$ tag algorithm uses a neural network which combines parameters sensitive to the displaced decay vertices of the B hadrons. A typical operating point in D0 top analyses achieves a $b$ jet efficiency of $\sim 54 \%$ and light flavor mistag rate of $\sim 1 \%$. The multijet background is determined as described above and contributions from other background sources in the inclusive sample are estimated by multiplying events by their probability to be $b$-tagged. The latter is parameterized for jet $E_{T}$ and $\eta$ using MC samples with corrections for object reconstruction differences between data and $\mathrm{MC}$ and for the estimated $t \bar{t}$ content of the sample. The lat- 
ter depends on $\sigma_{t \bar{t}}$ and so the process is repeated iteratively allowing the $t \bar{t}$ cross section estimate to vary until the result stabilizes. The result obtained is $\sigma_{t \bar{t}}=8.05 \pm 0.54$ (stat) \pm 0.7 (syst) \pm 0.49 (lumi) pb. The combined result is: $\sigma_{t \bar{t}}=7.42 \pm 0.53$ (stat) \pm 0.46 (syst) \pm 0.45 (lumi) pb. The main sources of systematic uncertainty are the selection efficiency, b-tagging, JES and MC modeling which each contribute $\sim 0.2 \mathrm{pb}$.

CDF has used secondary vertex $(S V X)$, Jet Probability $(J P B)$, and Soft Lepton $\left(S L T_{\mu}\right) b$-tag algorithms as well as kinematic likelihoods in Run 2 measurements of $\sigma_{t \bar{t}}$. The $J P B$ algorithm was used with a $318 \mathrm{pb}^{-1}$ dataset [158] requiring at least one tight $\left(P_{J} \leq 1 \%\right.$ ) b-tag to obtain $\sigma_{t \bar{t}}=$ $8.9 \pm 1.0$ (stat) ${ }_{-1.0}^{+1.1}$ (syst) pb for $M_{t}=178 \mathrm{GeV} / \mathrm{c}^{2}$. The $S V X$ algorithm was used with a comparable dataset [187] to obtain $\sigma_{t \bar{t}}=5.8 \pm 1.2$ (stat) ${ }_{-0.7}^{+0.9}$ (syst) pb. The $S L T_{\mu}$ algorithm described briefly in Section 4.3 was recently applied to $2 \mathrm{fb}^{-1}$ of data [188]. This analysis makes use of a new way of measuring the dominant background of fake muons in $W+$ jets from decay in flight and punch-through hadrons. First, kaons, pions and protons are identified by reconstructing $D^{*+} \rightarrow D^{o} \pi^{+} \rightarrow K^{-} \pi^{+} \pi^{+}$, and $\Lambda \rightarrow p \pi^{-}$decays (and their conjugates) using data collected with the SVT trigger. The muon fake rate is then measured for each particle type in bins of $p_{T}$ and $\eta$ and convoluted with the corresponding probabilities in these bins for the various particle types to appear in $W+$ jets events as taken from simulation. They obtain $\sigma_{t \bar{t}}=9.1 \pm 1.6 \mathrm{pb}$.

The most recent and the most precise $t \bar{t}$ production cross section measurements are performed by CDF with nearly $2.8 \mathrm{fb}^{-1}$ of data where the uncertainty is limited by the systematic uncertainty which in turn is dominated by the $5.8 \%$ uncertainty on the integrated luminosity. To overcome this hurdle the ratio $\mathcal{R} \equiv \sigma_{t \bar{t}} / \sigma_{Z}$ is measured using $\ell+$ jets events selected in a fairly standard way. The $t \bar{t}$ and $Z$ cross sections are then measured using events collected with identical triggers. The $t \bar{t}$ events are discriminated from backgrounds using either an artificial neural network $(A N N)$ [189] based on kinematic and topological variables like those used by D0, or by means of the secondary vertex $b$-tag algorithm [190]. The dominant $W+$ jets background is modeled in the $A N N$ study using simulated samples of $W+n$ jets with $n=0,1, \ldots, 4$ partons. In the $S V X$ study the $W+j$ jets background has to be broken into separate heavy flavor $(H F)$ and light flavor $(L F)$ jet categories. Because $W+H F$ events are poorly modeled in Monte Carlo, a data-driven correction factor is calculated in a non-signal region and used to estimate the amount of $W+H F$ in the signal region. $W+L F$ events only enter the final sample when a light flavor jet is mistagged. This background is estimated by applying a parameterization of the mistag rate from jet data to events that pass selections except for a secondary vertex tag. For the non- $W$ background in both analyses, the $\mathbb{E}_{T}$ distribution in data is fit to templates for non- $W$ events (taken for instance from dijet triggers passing all selection requirements except the $\mathbb{E}_{T}$ requirement) and a template for $t \bar{t}$ signal from simulation. The result for the $S V X$ analysis is $\sigma_{t \bar{t}}=7.1 \pm 0.4$ (stat) \pm 0.6 (syst) \pm 0.4 (lumi) pb. The inclusive $Z / \gamma^{*}$ cross section is then measured in the range $66 \leq M_{\ell \ell} \leq 116 \mathrm{GeV}$ using consistent triggers and definitions of leptons as for the $t \bar{t}$ cross section to obtain: $\operatorname{Br}\left(Z / \gamma^{*} \rightarrow \ell \ell\right) \times \sigma_{\left(Z / \gamma^{*} \rightarrow \ell\right)}=253.5 \pm 1.1$ (stat) \pm 4.5 (syst) \pm 14.9 (lumi) pb Together with the $t \bar{t}$ result one obtains $1 / \mathcal{R}=35.7 \pm 2.0$ (stat) \pm 3.2 (syst), where $\mathcal{R}$ is the ratio of $t \bar{t}$ to $Z$ production. Multiplying $\mathcal{R}$ by the current theoretical value for the $Z$ cross-section $\left(\sigma_{z}=251.3 \pm 5.0 \mathrm{pb}\right)$ one obtains $\sigma_{t \bar{t}}=7.0 \pm 0.4$ (stat) \pm 0.6 (syst) \pm 0.1 (theory) pb. A similar

procedure was carried out for the $A N N$ analysis to obtain $1 / \mathcal{R}=36.5_{-2.3}^{+2.1}$ (stat) ${ }_{-2.0}^{+1.9}$ (syst) and $\sigma_{t \bar{t}}=6.89 \pm 0.41$ (stat) ${ }_{-0.37}^{+0.41}$ (syst) \pm 0.14 (theory) pb.

\subsection{Measurements of $\sigma_{t \bar{t}}$ in the All-Hadronic Channel}

Despite having the largest branching ratio, the absence of leptons in the all-hadronic channel makes it the most difficult of all channels in which to isolate $t \bar{t}$. Since the first observations of $t \bar{t}$ in the allhadronic channel in Run 1 by CDF [191] and D0 [192]. both experiments have continued to improve their strategies for approaching this challenging measurement. D0 measured $\sigma_{t \bar{t}}$ with $405 \mathrm{pb}^{-1}$ in Run 2 , 
selecting events with $\geq 6$ jets, employing "loose" and "tight" secondary vertex $b$-tagging corresponding to requirements on decay length significance $L_{2 D} / \sigma_{L_{2 d}}>5$ or 7 , respectively. Events were required to have at least one tight or two loose tags. Single and double tagged events were treated separately. A neural network was used to estimate the $t \bar{t}$ content of the samples in order to extract a cross section. The neural network used a variety of variables to distinguish $t \bar{t}$ from QCD multijets which is the only relevant background. The 6 variables used include ones that are similar to those discussed previously in the context of other kinematic discriminant analyses but also included a mass $\chi^{2}$ variable $\mathcal{M}$ which utilized the $W$ and top quark mass constraints associated with $t \bar{t}$ events. The combined cross section is found to be $\sigma_{t \bar{t}}=4.5_{-1.9}^{+2.0}$ (stat) ${ }_{-1.1}^{+1.4}$ (syst) \pm 0.3 (lumi) pb.

More recently CDF has performed a measurement with $1.02 \mathrm{fb}^{-1}$ of data using a basic event selection of 6 or more jets, of which at least one is $b$-tagged, and kinematic requirements imposed by means of a neural network [163]. The neural network improves the $S / B$ of the pre-tagged event sample by $60 \%$ to $1 / 12$ as compared to the previous cut-based measurement [164]. The multijet background is estimated from data using $t \bar{t}$-depleted control samples such as multijet events with exactly four jets with an estimated $t \bar{t}$ fraction of roughly $1 / 3600$. These events are used to parameterize the per-jet $b$-tag rate in multijet events, comprising both real heavy flavor tags and mistags. The parameterization is then validated on multijet events with higher numbers of jets prior to selection with the neuralnetwork, which is at a stage where the samples are dominated by background. After the neuralnetwork and $b$-tag requirements, 926 tags are observed in 772 events for which the background is estimated to account for $567 \pm 28$ of the tags. The cross section obtained with the selected sample is $\sigma_{t \bar{t}}=8.3 \pm 1.0$ (stat) ${ }_{-1.5}^{+2.0}$ (syst) \pm 0.5 (lumi) pb

\subsection{Combined Cross Sections from the Tevatron and Prospects for the LHC}

CDF and D0 have both recently released $t \bar{t}$ production cross section values obtained by combining results from various analyses. D0 combines results from the $\ell+j e t s, \ell \ell$ and $\ell \tau$ final states $(\ell=e, \mu)$ measured with $1 \mathrm{fb}^{-1}$ of Run 2 data 193 , with an assumed top mass value of $M_{t}=170 \mathrm{GeV} / \mathrm{c}^{2}$. The combined cross section is calculated with a joint likelihood function that is the product of Poisson probabilities for 14 non-overlapping subsamples of events. Additional Poisson terms representing backgrounds estimated separately in each subsample are also included. Systematic uncertainties are included as nuisance parameters with Gaussian distributions centered at zero and widths corresponding to a single standard deviation in the parameter uncertainty. The final result is:

$$
\sigma_{t \bar{t}}=8.18_{-0.87}^{+0.98} \mathrm{pb} \quad \text { D0 } 1 \mathrm{fb}^{-1}
$$

CDF combines five preliminary measurements performed with $2.8 \mathrm{fb}^{-1}$ of data [101]. One of the measurements is performed in the dilepton channel while the other four are in the $\ell+j e t s$ channel using different methods to discriminate against background including $A N N, S V X$ and $S L T_{\mu}$ already discussed earlier. The fourth measurement uses a Soft Electron $b$-tag $\left(S L T_{e}\right)$. The combination uses the BLUE technique [194] taking into account statistical and systematic correlations. The resulting cross section is:

$$
\sigma_{t \bar{t}}=7.02 \pm 0.63 \mathrm{pb} \quad \mathrm{CDF} 2.8 \mathrm{fb}^{-1}
$$

These measurements are in good agreement with theoretical expectations and have uncertainties comparable to those associated with the theoretical calculations. Reaching this level of accuracy has required many years during which statistically significant signal samples were accumulated and improved methods of analysis were developed and exploited. The ATLAS and CMS experiments have paid close attention to the Tevatron $t \bar{t}$ studies over the years and now contain many of the seasoned Tevatron experts. As discussed in Section 4.2 the LHC will be a top factory, producing huge numbers of $t \bar{t}$ 
events. The ATLAS and CMS collaborations are currently preparing to study events in early data taking. These studies have concentrated on the leptonic final states with an initial goal of assessing the $t \bar{t}$ production cross section at $10 \mathrm{TeV}$ as a background to new physics searches. ATLAS has studied the discrimination afforded by reconstruction of the hadronically decaying top quark in the $\ell+j e t s$ channel with and without $b$ tagging [119] which may not be available immediately at the start of data-taking. The dilepton channel without $b$ tagging has also been studied by ATLAS as well as CMS [79]. Once detectors and software are well understood, and large event samples are collected, the LHC experiments will begin to produce results comparable in quality to the Tevatron results but the environment of the LHC is different from that of the Tevatron and several new elements will require new adaptations. For instance, there will be substantially more jets in $t \bar{t}$ and $W / Z+$ jets events. For $E_{T} \geq 15 \mathrm{GeV}$, the cross section for $t \bar{t}+\geq 1$ jet saturates the total $t \bar{t}$ production cross section. Additionally, while it is true that $\sigma_{t \bar{t}}$ increases 100 -fold relative to the Tevatron while the inclusive $W$ and $Z$ cross sections increase by roughly a factor of 5 , the cross sections for $W / Z$ production in association with four or more jets [195] increase as much or more than $\sigma_{t \bar{t}}$ production and it is these events that are the backgrounds to $t \bar{t}$ in the $l+$ jets channels, for instance. All in all, the study of $t \bar{t}$ production will continue to be challenging at the LHC, but it will also continue to be rewarding and potentially crucial to the discovery of new physics.

\section{Measurement of Single Top-Quark Production}

While $t \bar{t}$-pair production via the strong interaction is the main source of top quarks at the TEVATRON and the LHC, the SM also predicts the production of single top-quarks via charged-current weak interactions, in which a virtual $W$ boson is either exchanged in the $t$-channel ( $t q \bar{b}$ production) or in the $s$-channel ( $t \bar{b}$ production), see section $[3.2$. At the LHC associated $W t$ production will also be important. While Run I and early Run II searches for single top-quarks at CDF [196, 197, 198] and DØ [199, 200, 201, 202] could only set upper limits on the production cross section, recent analyses, using more data and advanced analysis techniques, have found first evidence for singly produced top quarks [203, 204, 205]. Updates of theses analyses to larger data sets lead to the observation of single top-quark production at the level of five standard deviations in March 2009 [206, 207].

\subsection{Observation of Single Top Quarks at CDF and DØ}

The main thrust of these analyses was to establish evidence for single top-quarks, considering both production modes relevant at the Tevatron, $t$-channel and $s$-channel, as one single-top signal. The ratio of $t$-channel to $s$-channel events is assumed to be given by the SM. That is why, this search strategy is often referred to as combined search. By measuring the inclusive single top-quark cross section and using theory predictions one can deduce the absolute value of the CKM matrix element $\left|V_{t b}\right|$, without assuming there are only three generations of quarks. If one would measure $\left|V_{t b}\right|$ to be significantly smaller than one this would be a strong indication for a fourth generation of quarks or other effects beyond the SM [208, 209].

Single top-quark events feature a $W b \bar{b}$ (s-channel) or $W b q \bar{b}$ ( $t$-channel) partonic final state. The $W$ boson originating from the top-quark decay is reconstructed in the leptonic decay modes $e \nu_{e}$ or $\mu \nu_{\mu}$, while hadronic $W$ decays and decays to $\tau \nu_{\tau}$ are not considered because of large backgrounds from QCDinduced jet production. The quarks from the hard scattering process manifest themselves as hadronic jets with transverse momentum. Additional jets may arise from hard gluon radiation in the initial or final state. The experimental signature of SM single top-quarks is therefore given by one isolated high- $p_{\mathrm{T}}$ charged lepton, large missing transverse energy $\left(\mathbb{E}_{T}\right)$, and two or three high- $E_{T}$ jets.

At $\mathrm{D} \varnothing$, various trigger algorithms have been used requiring an electron or muon candidate and one 
or two jets. The backbone of the CDF analyses are collision data triggered by central electron or muon candidates. The lepton coverage is extended by one additional trigger path which requires large $\mathbb{E}_{T}$ and an energetic electromagnetic cluster in the forward calorimeter, and one path asking for large $\mathbb{E}_{T}$ and two jets. The latter one targets muon events in which a muon candidate could not be established at trigger level, but is reconstructable offline.

Event Selection At CDF, the offline event selection requires exactly one isolated electron with $E_{T}>20 \mathrm{GeV}$ or one isolated muon with $p_{T}>20 \mathrm{GeV} / c$. Electrons and muons are reconstructed up to $|\eta|<1$.6. D $\varnothing$ applies slightly lower $p_{T}$ thresholds and asks for electrons with $E_{T}>15 \mathrm{GeV}$ and muons with $p_{T}>18 \mathrm{GeV} / c$. Electrons are identified in the central region up to $|\eta|<1.1$ and muons up to $|\eta|<2.0$. In order to reduce the $Z+$ jets, $t \bar{t}$, and diboson backgrounds, events with a second lepton candidate are rejected by both collaborations. Cosmic ray and photon conversion events are identified and removed. Neutrinos from $W$-boson decay remain undetected and cause an imbalance in the transverse momentum sum. CDF selects events with $\mathbb{E}_{T}>25 \mathrm{GeV}$, while DØ applies the cut $15 \mathrm{GeV}<\mathbb{E}_{T}<200 \mathrm{GeV}$. QCD-multijet background without a leptonic $W$ decay is further minimized with additional requirements on, e.g., the angle between the direction of $\mathbb{E}_{T}$ and the lepton candidate, thereby removing events in which the lepton and the jet are in a back-to-back configuration. Reducing QCD-multijet events to a level of just a few percent is crucial because it is very difficult to model the full event kinematics of these misidentified events.

Hadronic jets are identified at CDF by a fixed-cone algorithm with radius $\Delta R \equiv \sqrt{(\Delta \eta)^{2}+(\Delta \phi)^{2}}=$ 0.4, while $\mathrm{D} \varnothing$ uses a midpoint-cone algorithm with $\Delta R=0.5$. Jet-energy corrections are applied to account for instrumental effects [210] and convert reconstructed jet energies to particle-level energies. Jets with the same $\eta$ and $\phi$ as a reconstructed electron are removed from the list of jets to avoid double-counting physics objects. CDF asks for two or three jets with $|\eta|<2.8$ and $E_{T}>20 \mathrm{GeV}$. The DØ analyses use events with two, three, or four jets with $E_{T}>15 \mathrm{GeV}$ and $|\eta|<3.4$, but there are additional requirements on the leading and subleading jet. The leading jet has to have $E_{T}>25 \mathrm{GeV}$ and $|\eta|<2.5$, the second leading one $E_{T}>20 \mathrm{GeV}$. Both collaborations further require at least one of the jets to be identified as originating from a $b$ quark. For a jet to be $b$ tagged at CDF it must contain a reconstructed secondary vertex consistent with the decay of a $b$ hadron [168]. D $\varnothing$ utilizes an advanced $b$-tagging algorithm based on neural networks (NN) [211]. To be subjected to the tagging algorithm, the jets have to contain at least two tracks that fulfill some minimum quality criteria and point to a common origin. Those jets are considered to be taggable. The NN uses various input variables to discriminate $b$-quark jets from other jets, such as the impact parameter significances of the tracks associated to the jet, the mass and the decay length significance of the secondary vertex, and the number of tracks used to reconstruct the secondary vertex. For a jet to be considered a $b$-quark jet the output of the NN has to be above a certain threshold. The average probability for a light-quark jet to be misidentified as a $b$-quark jet at this operating point is $0.5 \%$, while the average tagging efficiency for true $b$-quark jets with $|\eta|<2.4$ is $47 \%$.

Background Estimation The expected background event rates are obtained from a compound model comprising simulated events, theoretical cross sections, and normalizations in background-dominated control samples. The basic strategy is to normalize the main component, the $W+$ jets rate, to the observed yield before applying the $b$-tagging algorithm. This sample of events is the so-called pretag data set. The idea behind this is that the samples of simulated $W+$ jets events describe the event kinematics well, but do not predict the event rate correctly, and therefore it is sufficient to scale up the event rate by a constant factor. For the normalization in the pretag data to work the contributions to this data set which are not due to $W+$ jets have to be determined. The $t \bar{t}$ contribution is estimated by normalizing samples of simulated events - CDF uses PYTHIA [212], DØ ALPGEN [174] - to NLO cross sections. CDF estimates small contributions of a few electroweak processes, diboson production $(W W$, 
Table 4: Numbers of expected and observed lepton+jets events in the CDF and D $\varnothing$ single top-quark analyses using $3.2 \mathrm{fb}^{-1}$ or $2.3 \mathrm{fb}^{-1}$, respectively. The total prediction is in some cases not equal to the sum of the processes because of round-off effects.

\begin{tabular}{lccccc}
\hline \multirow{2}{*}{ Process } & $W+2$ jets & $W+3$ jets & $W+2$ jets & $W+3$ jets & $W+4$ jets \\
\hline$t q b+t b$ & $146 \pm 21$ & $45 \pm 7$ & $139 \pm 18$ & $63 \pm 10$ & $21 \pm 5$ \\
$W+$ jets & $1701 \pm 372$ & $503 \pm 109$ & $1829 \pm 161$ & $637 \pm 61$ & $180 \pm 18$ \\
$Z+$ jets / dibosons & $125 \pm 9$ & $46 \pm 3$ & $229 \pm 38$ & $85 \pm 17$ & $26 \pm 7$ \\
$t \bar{t}$ & $204 \pm 30$ & $482 \pm 70$ & $222 \pm 35$ & $436 \pm 66$ & $484 \pm 71$ \\
QCD multijets & $90 \pm 36$ & $35 \pm 14$ & $196 \pm 50$ & $73 \pm 17$ & $30 \pm 6$ \\
\hline Total prediction & $2265 \pm 375$ & $1112 \pm 130$ & $2615 \pm 192$ & $1294 \pm 107$ & $742 \pm 80$ \\
Observation in data & 2229 & 1086 & 2579 & 1216 & 724 \\
\hline
\end{tabular}

$W Z, Z Z)$ and $Z+$ jets in the same way, while $\mathrm{D} \varnothing$ incorporates those in the $W+$ jets estimate. However, the main challenge in understanding the pretag data set is to estimate the fraction of QCD-multijet events. CDF tackles this challenge by removing the cut on $\mathbb{E}_{T}$ and fitting the $\mathbb{E}_{T}$ distribution. The region of $\mathbb{E}_{T}<25 \mathrm{GeV}$ is dominated by QCD-multijet events and provides a normalization for this event class in the signal region. DØ uses the so-called matrix method to determine the contribution of QCD-multijet events. This method links the number of events with real and misidentified leptons in the pretag data set to the numbers in a control sample with relaxed lepton identification cuts by applying cut efficiencies that are derived in specific calibration data sets, i.e. $Z \rightarrow \ell \ell$ data or events with $\mathbb{E}_{T}<10 \mathrm{GeV}$. The factors to normalize the $W+$ jets background model to the observed pretag data are derived separately for each $W+n$ jets sample and for the different lepton categories. At $\mathrm{D} \varnothing$ these scale factors vary across the samples from $0.9 \pm 0.2$ to $1.6 \pm 0.4$. Both collaborations use the LO matrix-element generator ALPGEN [174] combined with parton showering and the underlying event model by PYTHia [212]. To properly describe the flavor composition in $W+$ jets data, CDF scales up the fraction of $W b \bar{b}$ and $W c \bar{c}$ events predicted by ALPGEN by a factor of $1.4 \pm 0.4$, as demonstrated by studies in the $W+1$ jet sample where one jet is identified as a $b$-quark jet. D $\varnothing$ uses a correction factor of 1.47 for $W b \bar{b}$ and $W c \bar{c}$, and 1.38 for the $W c j$ contribution.

$\mathrm{D} \varnothing$ derives the event yield in the $b$-tagged data sets from samples of simulated pretagged events after proper normalization by applying tag-rate functions derived from control samples. While D $\varnothing$ uses the tag-rate parameterization for all flavors, CDF applies the $b$-tagging algorithm to $b$-quark jets and $c$-quark jets in simulated events. The rates of tagged heavy flavor jets are subsequently corrected by a constant factor of $0.95 \pm 0.05$. The rate of misidentified light-quark jets at CDF is obtained from mistag-rate functions applied to the observed pretag data subtracting all contributions which are not due to $W+$ light jets.

CDF simulates single-top events using the LO matrix-element generator MADEVENT [213]. The two $t$-channel processes in Fig. \&[(b)] and in Fig. \&[(a) are produced and combined [214] to one sample to match the event kinematics as predicted by a fully differential NLO calculation [109]. D $\varnothing$ uses the Monte Carlo generator COMPHEP-SIngletop [215, 216] which has the matching between the two $t$-channel processes built in.

The resulting prediction and the observed event yields are given in Table 4. DØ finds a total acceptance of $(2.1 \pm 0.3) \%$ for the $t$-channel process and $(3.2 \pm 0.4) \%$ for the $s$-channel. The CDF analyses use an acceptance of $(1.8 \pm 0.3) \%$ for the $t$-channel and $(2.7 \pm 0.4) \%$ for the $s$-channel. In the lepton+jets data set corresponding to $3.2 \mathrm{fb}^{-1}$ CDF expects $191 \pm 28$ single top-quark events, while the $\mathrm{D} \varnothing$ expectation is $223 \pm 32$ signal events in $2.3 \mathrm{fb}^{-1}$. The dominating background process is $W b \bar{b}$, 


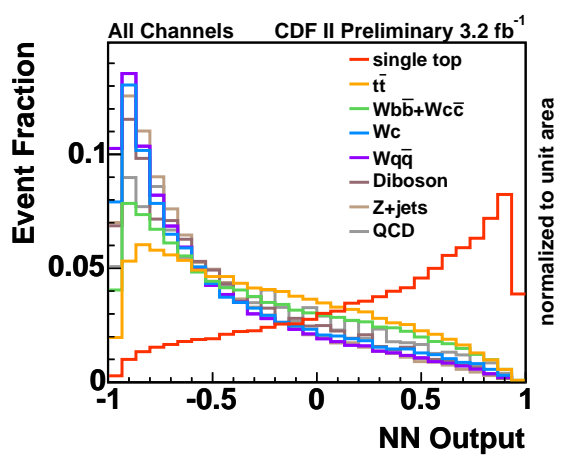

(a)

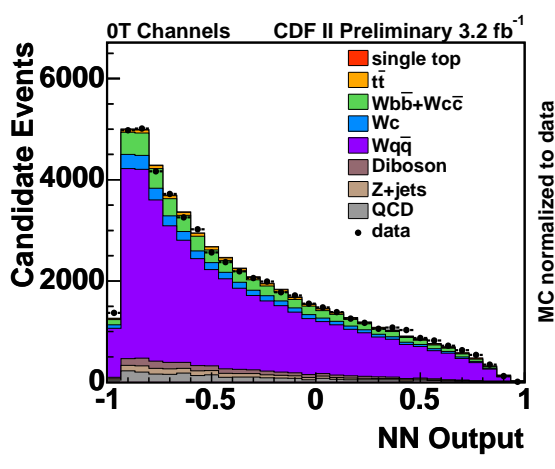

(b)

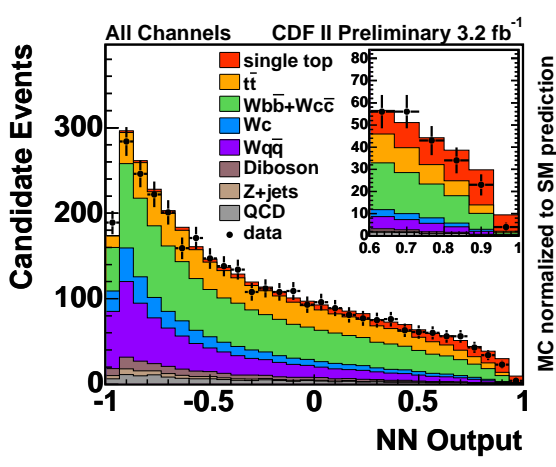

(c)

Figure 11: CDF NN analysis. (a) Normalized shapes of the NN output. (b) NN output on the untagged data set for validation. (c) NN output on the selected data set compared to the SM prediction.

followed by misidentified $W+$ light-quark jet events, $W c \bar{c}$, and $W c j$. In the $W+3$ jets data set $t \bar{t}$ is the most important background.

At CDF, an additional analysis investigates the $\mathbb{E}_{T}+$ jets data set which is disjoint to the lepton + jets data set mentioned above. The analysis selects events in which the $W$ boson decays into $\tau$ leptons and those in which the electron or muon are not identified. The main background in the $\mathbb{E}_{T}+$ jets channel is QCD-multijet production which is reduced by means of a NN discriminant that is computed using 15 kinematic variables. A cut on this discriminant reduces the background by $77 \%$, while the single top-quark signal is only diminished by $9 \%$. In a data set corresponding to $2.1 \mathrm{fb}^{-1}$ CDF selects 1411 candidate events. The expectation value of single top-quark events is $64 \pm 10$.

Multivariate Analyses Even though the single top-quark production cross section is predicted [108, 111 to amount to about $40 \%$ of the $t \bar{t}$ cross section, the signal has been obscured for a long time due to the very challenging background situation. After the cut-based event selection sketched above the signal-to-background ratio is only about 5 to $6 \%$. Further kinematic cuts on the event topology proofed to be prohibitive, since the number of signal events in the resulting data set would become too small. Given this challenging situation the analysis groups in both TEVATRON collaborations turned towards the use of multivariate techniques, in order to exploit as much information about the observed events as possible. The explored techniques comprise artificial neural networks (NN), LO matrix elements $(\mathrm{ME})$, boosted decision trees (BDT), and likelihood ratios. All these multivariate techniques aim at maximizing the separation between the single top-quark signal and the backgrounds by combining the information contained in several discriminating variables to one powerful discriminant. An example of these discriminants is shown in Fig. 11](a). To ascertain that these advanced methods work reliably, extensive checks have been undertaken. In a first step, it is important to check the modeling of input variables. This can be done on the set of selected events, but these checks are statistically limited. It is therefore more meaningful to check the modeling of the event kinematics in the untagged data set which comprises twenty to fifty times more events and consists of events in which at least one jet is taggable, but no jet is actually identified as a $b$-quark jet. A very important further check is to compute the discriminant on the untagged data set, which is completely dominated by background, and compare the observed distribution to the one obtained from the background model, see Fig. [11](b), Finally, after additional tests of robustness of the method and further checks of the background model have been passed, the analysis techniques are applied to the signal sample. Fig. 11](c) shows the NN discriminant at CDF in the lepton+jets data set. The observed distribution is compared to the expectation based on the background model and the SM prediction for the rate of single top-quark events. 


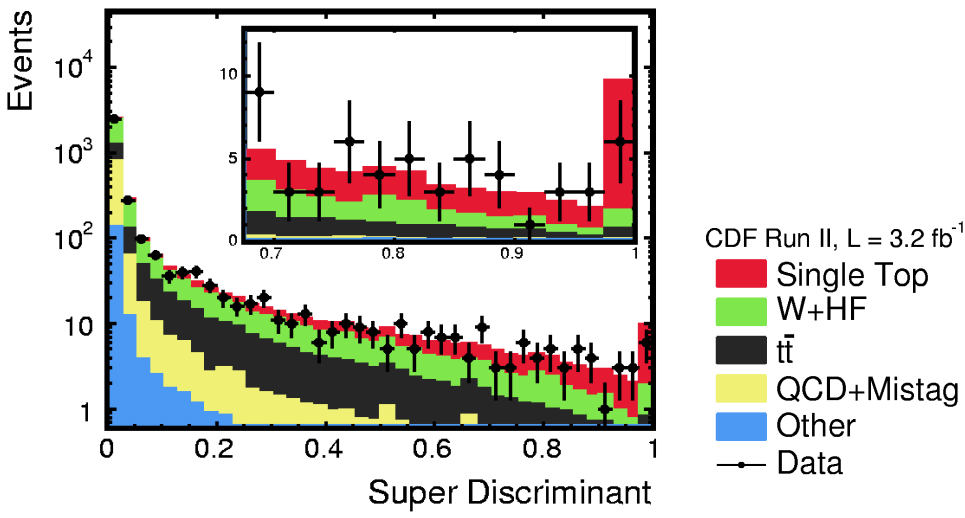

(a)

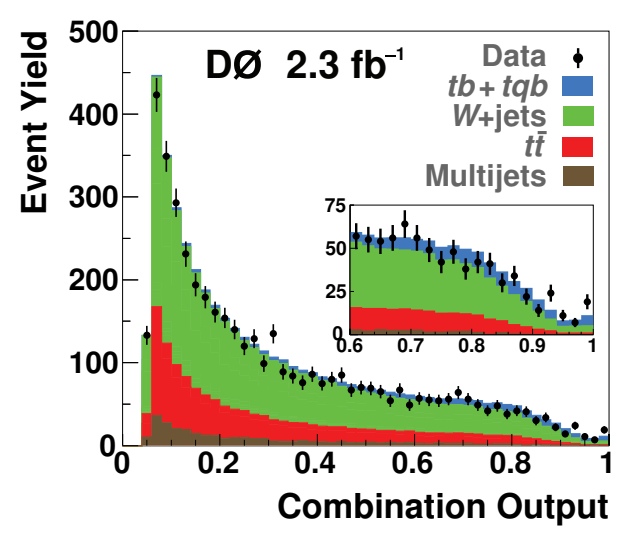

(b)

Figure 12: Distributions of super-discriminants, (a) at CDF.(b) at DØ. The observed data are compared to the SM prediction.

Even though all analyses run on the lepton+jets data set share the same events they are not fully correlated, the typical correlation being about 70\%. It is therefore worthwhile to combine the analyses. Both collaborations do this by computing a super-discriminant which takes the individual discriminants as input and combines them based on NN techniques. The resulting distributions are displayed in Fig. 12.

The templates of the discriminant distributions for the different processes, see e.g. Fig. 11](a), are compared to the observed data distribution using a Bayesian maximum likelihood technique. Systematic uncertainties arise from uncertainties in the jet-energy corrections, the modeling of initial and final state gluon radiation, the factorization scale used to generate the simulated events, the modeling of the underlying event and the hadronization process, and efficiencies for $b$-tagging, lepton identification and trigger. The effects of these uncertainties on the background rates, the signal acceptance and detection efficiency and the shape of the discriminant templates are investigated with dedicated Monte Carlo samples or by studying control samples of collision data, and then incorporated in the likelihood function in a parameterized form. The posterior probability density of the single top-quark cross section is then obtained by integrating over the parameters associated with the systematic uncertainties using Gaussian prior distributions and by applying a flat prior distribution to the signal cross section which is zero for negative values and one for positive ones. The cross sections measured by the various analyses and the combination are summarized in Table 5. CDF quotes the measured cross sections at $m_{t}=175 \mathrm{GeV} / c^{2}$, DØat $m_{t}=170 \mathrm{GeV} / c^{2}$. However, the acceptance correction due to $m_{t}$ are relatively small, namely $+0.02 \mathrm{pb} /\left(\mathrm{GeV} / c^{2}\right)$.

The significance of the expected and observed single-top signal is determined using a frequentist approach based on hundreds of millions of ensemble tests. While CDF uses the so-called $Q$-value as a test statistic, D $\varnothing$ uses the measured cross section. The $Q$-value is defined as the ratio of the posterior probability density for the observed data assuming the predicted single-top cross section over the posterior probability density assuming no single top-quarks to be present. Based on the chosen test statistic the collaborations calculate the probability ( $p$-value) to obtain the measured or a higher cross section under the assumption the observed data contain only background. The observed and expected significances are expressed in terms of standard deviations of a Gaussian distribution and given in Table 5. It is interesting to note that the CDF lepton+jets analyses are all below the theoretically expected cross section of $2.9 \pm 0.4 \mathrm{pb}$, while all $\mathrm{D} \varnothing$ measurements indicate a value above the prediction. Since the analyses in each collaboration use the same events, they are highly correlated with a correlation coefficient between $60 \%$ or $70 \%$. In summary, both collaborations have observed 
Table 5: Cross sections measured by the multivariate analyses searching for single top-quarks. The quoted cross sections are the sum of $t$-channel and $s$-channel cross sections for top quark and antitop quark production. Additionally, the expected and observed significance in standard deviations (SD) are given. The theoretical prediction at NLO is quoted for $m_{t}=175 \mathrm{GeV} / c^{2}$.

\begin{tabular}{|c|c|c|c|}
\hline Analysis & $\begin{array}{c}\text { Cross Section } \\
{[\mathrm{pb}]}\end{array}$ & $\begin{array}{l}\text { Expected Significance } \\
\text { [standard deviations] }\end{array}$ & $\begin{array}{l}\text { Observed Significance } \\
\text { [standard deviations] }\end{array}$ \\
\hline \multicolumn{4}{|c|}{$\mathrm{CDF}$} \\
\hline Neural Networks (NN) & $1.8_{-0.6}^{+0.6}$ & 5.2 & 3.5 \\
\hline Boosted Decision Tree (BDT) & $2.1_{-0.6}^{+0.7}$ & 5.2 & 3.5 \\
\hline Matrix Elements (ME) & $2.5_{-0.6}^{+0.7}$ & 4.9 & 4.3 \\
\hline Likelihood Function & $1.6_{-0.7}^{+0.8}$ & 4.0 & 2.4 \\
\hline$s$-Channel Likelihood & $1.5_{+0.8}^{+0.9}$ & 1.1 & 2.0 \\
\hline Super-Discriminant & $2.1_{-0.5}^{+0.6}$ & $>5.9$ & 4.8 \\
\hline $\mathbb{E}_{T}+$ Jets & $4.9_{-2.2}^{+2.5}$ & 1.4 & 2.1 \\
\hline Combined & $2.3_{-0.5}^{+0.6}$ & $>5.9$ & 5.0 \\
\hline \multicolumn{4}{|c|}{$\mathrm{D} \varnothing$} \\
\hline Boosted Decision Tree (BDT) & $3.7_{-0.8}^{+1.0}$ & 4.3 & 4.6 \\
\hline Neural Networks (NN) & $4.7_{-0.9}^{+1.2}$ & 4.1 & 5.2 \\
\hline Matrix Elements (ME) & $4.3_{-1.2}^{+1.0}$ & 4.1 & 4.9 \\
\hline Combined & $3.9 \pm 0.9$ & 4.5 & 5.0 \\
\hline Theory $\left(\mathrm{NLO}, m_{t}=175 \mathrm{GeV} / c^{2}\right)$ & $2.9 \pm 0.4$ & - & - \\
\hline
\end{tabular}

single top-quark production via the weak interaction at the level of five standard deviations.

Determination of $\left|\mathbf{V}_{\mathbf{t b}}\right|$ The measured single top-quark production cross sections can be used to determine the absolute value of the CKM-matrix element $V_{t b}$, if one assumes $V_{t b} \gg V_{t s}, V_{t b} \gg V_{t d}$, and a SM-like left-handed coupling at the $W t b$ vertex. Contrary to indirect determinations in the framework of flavor physics [11] the extraction of $V_{t b}$ via single top-quark production does not assume unitarity of the CKM matrix and is thereby sensitive to a fourth generation of quarks. The assumption of $V_{t s}$ and $V_{t d}$ being small compared to $V_{t b}$ enters on the production side, since top quarks can also be produced by $W t s$ and $W t d$ vertices, and in top-quark decay. The phenomenological analysis of [208] as well as the measurement of $R_{b}$, see section 8.1, indicate that this assumption is well justified.

To determine $\left|V_{t b}\right|$ the analysts divide the measured single top-quark cross section by the predicted value, which assumes $\left|V_{t b}\right|=1$, and take the square root. CDF obtains $\left|V_{t b}\right|=0.91 \pm 0.11$ (stat + syst) \pm 0.07 (theory) and $\left|V_{t b}\right|>0.71$ at the $95 \%$ C.L. DØ finds $\left|V_{t b}\right|=1.07 \pm 0.12$ and $\left|V_{t b}\right|>0.78$ at the $95 \%$ C.L. The lower limits assume a flat prior for $\left|V_{t b}\right|^{2}$ in the interval $[0,1]$.

\subsection{Separation of t-channel and s-channel Events}

In addition to the combined-search analyses described in the previous section CDF and D $\varnothing$ have also attempted to separate $t$-channel and $s$-channel single top-quark events. Both processes are treated as independent, i.e., the assumption of the ratio of the two cross sections to be given by the SM 


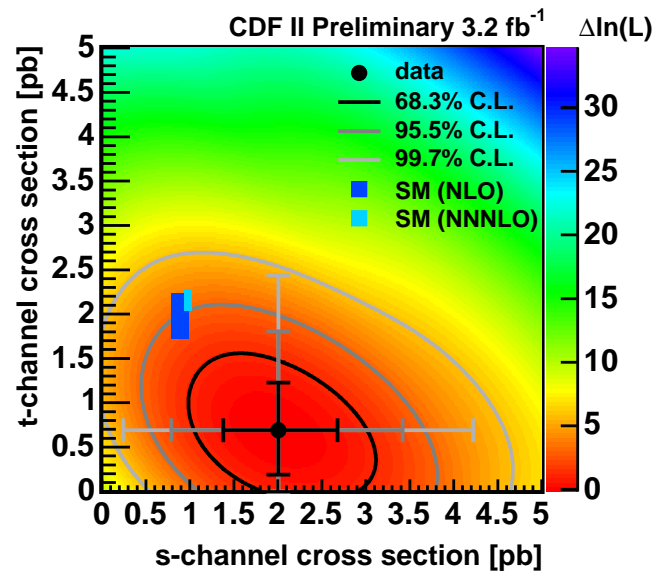

(a)

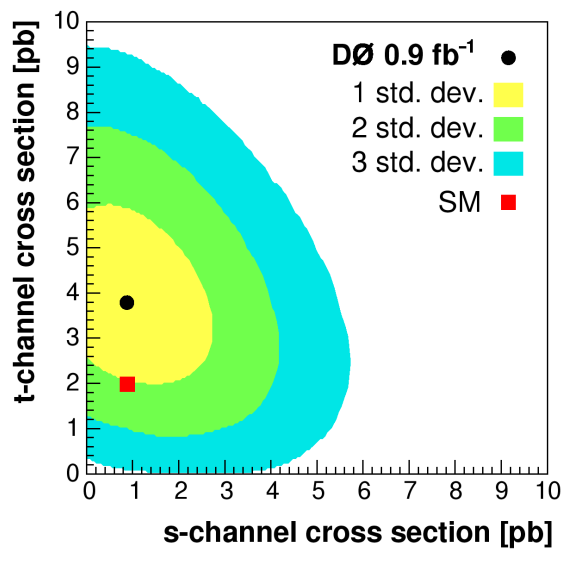

(b)

Figure 13: Results of the analyses separating $t$-channel $(t q \bar{b})$ and $s$-channel $(t \bar{b})$ events. The figures show the contours of $t$-channel versus the $s$-channel cross section, (a) obtained from the NN separate-search analysis at CDF, (b) obtained from the BDT analysis at D $\varnothing$. The separate search of DØ uses only $0.9 \mathrm{fb}^{-1}$.

is dropped. The separation of the two channels is important because they are subject to different systematic uncertainties. The $t$-channel cross section, for example, is quite sensitive to the $b$-quark PDF, respectively, the gluon PDF. In addition, the two channels also exhibit a different sensitivity to physics beyond the SM [217]. In Fig. 13 the resulting contours in cross section space are shown. CDF measures $\sigma_{t q \bar{b}}=0.7_{-0.5}^{+0.5} \mathrm{pb}$ and $\sigma_{t \bar{b}}=2.0_{-0.6}^{+0.7} \mathrm{pb}$. The quoted uncertainties include statistical and systematic components. The most probable values of the $\mathrm{D} \varnothing$ analysis are at $\sigma_{t q \bar{b}}=3.8 \mathrm{pb}$ and $\sigma_{t \bar{b}}=0.9 \mathrm{pb}[204]$. The values for the $t$-channel cross section are pointing in different directions. While CDF measures about $2 \sigma$ lower than the predicted value $\left(\sigma_{t q \bar{b}}=1.98_{-0.22}^{+0.28} \mathrm{pb}\right.$ at $\mathrm{NLO}$ and $\left.m_{t}=175 \mathrm{GeV} / c^{2}\right), \mathrm{D} \varnothing$ measures about $1 \sigma$ higher.

\subsection{Prospects for Single Top-Quark Measurements at the LHC}

Collisions at the LHC will be a copious source of top-quarks. Not only the $t \bar{t}$ cross section, but also the cross sections for single top-quark production will be a factor of 100 higher than at the TEVATRON. It will therefore be possible to not only measure cross sections, but also carefully study properties of single top-quarks in detail, like for example the polarization. The LHC experiments ATLAS and CMS have thoroughly studied the prospects of single top-quark measurements at the LHC [79, 119], which we will briefly summarize here.

Single top-quark analyses at ATLAS are based on a common preselection of events, which asks for an isolated electron or muon with $|\eta| \leq 2.5$ and $p_{T}>30 \mathrm{GeV} / c, \mathbb{E}_{T}>20 \mathrm{GeV}$, and at least two jets with $|\eta| \leq 5.0$ and $p_{T}>30 \mathrm{GeV} / c$, one of which has to be identified to originate from a $b$ quark [119]. Events with an additional isolated lepton with $p_{T}>10 \mathrm{GeV} / c$ are vetoed to reduce the number of $t \bar{t}$ dilepton events. Events with three or more additional jets with $p_{T}>15 \mathrm{GeV} / c$ are also removed.

After preselection, the analyses optimized for the different single top-quark channels follow separate branches. The selection of single-top t-channel candidate events dwells on the signature of a high$p_{T}$ light-quark jet in the forward direction, requiring $|\eta| \geq 2.5$. The $b$-tagged jet has to have $p_{T}>$ $50 \mathrm{GeV} / c$. Based on these cuts a signal-to-background ratio of $37 \%$ will be reached. With collision data corresponding to $1 \mathrm{fb}^{-1}$ ATLAS expects to measure $\left|V_{t b}\right|$ with a relative uncertainty of $\pm 12 \%$.

The discrimination of $s$-channel single top-quark events and $W t$ events will be much more challenging 
than measuring the $t$-channel cross section. ATLAS is therefore studying multivariate techniques to isolate those events. Evidence for $s$-channel production appears to be achievable at the $3 \sigma$ level with $30 \mathrm{fb}^{-1}$ of collision data, while evidence for $W t$ production may be reached with $10 \mathrm{fb}^{-1}$ using a BDT analysis.

Performance studies at CMS are based on sets of simulated events corresponding to $10 \mathrm{fb}^{-1}[79]$. The $t$-channel analysis asks for one isolated muon with $|\eta| \leq 2.1$ and $p_{T}>19 \mathrm{GeV} / c, \mathbb{E}_{T}>40 \mathrm{GeV}$, and two jets, one of which is identified as a $b$-quark jet. This $b$-tagged jet is required to have $|\eta|<$ 2.5 and $p_{T}>35 \mathrm{GeV} / c$, the light-quark jet must be in the forward direction with $|\eta|>2.5$ and $p_{T}>40 \mathrm{GeV} / c$. Further cuts are placed on the transverse mass of the reconstructed $W$ boson, $50<$ $M_{T}(W)<120 \mathrm{GeV} / c^{2}$, and the invariant mass of the reconstructed top-quark candidate, $110<M_{\ell \nu b}<$ $210 \mathrm{GeV} / c^{2}$. With these selection cuts CMS reaches a signal-to-background ratio of 1.34. The relative uncertainty on $\left|V_{t b}\right|$ is expected to be $5 \%$. For the $W t$-production mode the dilepton and semileptonic channels have been studied at CMS. The signal-to-background ratio is found to be 0.37 and 0.18 , respectively. The $s$-channel will be even more difficult yielding a signal-to-background ratio of 0.13.

\section{Top-Quark Mass Measurements}

The direct observation of the top quark in 1995 [46, 47] was anticipated since the $b$-quark was expected to have an isospin partner to ensure the viability of the Standard Model, and therefore not a big surprise. What was a surprise is the very large mass of the top-quark, almost 35 times the $b$-quark mass. The top-quark mass is a fundamental parameter in the Standard Model, and plays an important role in electroweak radiative corrections, and therefore in constraining the mass of the Higgs boson. A large value of the top-quark mass [50] indicates a strong Yukawa coupling to the Higgs, and could provide special insights in our understanding of electroweak symmetry breaking [218]. The top-quark mass could have a different origin than the masses of the other light quarks. Thus, precise measurements of the top-quark mass provide a crucial test of the consistency of the Standard Model and could indicate a strong hint for physics beyond the Standard Model. In doing that, it is important to measure and compare the top-quark mass in the different decay channels. Since all top mass measurements assume a sample composition of $t \bar{t}$ and Standard Model background events, any discrepancy among the measured top masses could indicate the presence of non-Standard Model events in the samples.

\subsection{Lepton+Jets Channel}

The top mass has been measured in the lepton+jets, dilepton and the all-jets channel by both CDF and $\mathrm{D} \varnothing$. At present, the most precise measurements come from the lepton+jets channel containing four or more jets and large missing $E_{T}$. The samples for the mass measurement are selected using topological (topo) or $b$-tagging methods. In this channel, four basic techniques are employed to extract the top mass. In the first, the so-called "template method" (TM) [219, 46, 47], an over-constrained (2C) kinematic fit is performed to the hypothesis $t \bar{t} \rightarrow W^{+} b W^{-} \bar{b} \rightarrow \ell \nu_{\ell} b q \bar{q}^{\prime} \bar{b}$ for each event, assuming that the four jets of highest $E_{T}$ originate from the four quarks in $t \bar{t}$ decay. There are 24 possible solutions reflecting the allowed assignment of the final-state quarks to jets and two possible solutions for the longitudinal momentum, $p_{z}$, of the neutrino when the $W$ mass constraint is imposed on the leptonic $W$ decay. The number of solutions is reduced to 12 when a jet with an identified secondary vertex is assigned as one of the $b$ quarks, and to 4 when the event has two such secondary vertices. A $\chi^{2}$ variable describes the agreement of the measurement with each possible solution under the $t \bar{t}$ hypothesis given jet-energy resolutions. The solution with the lowest $\chi^{2}$ is defined as the best choice, resulting in one value for the reconstructed top quark mass per event. The distribution of reconstructed top-quark mass from the data events is then compared to templates modeled from a mixture of signal and background 
distributions for a series of assumed top masses, see Fig. 14. The best fit value for $m_{t}$ and its uncertainty are obtained from a maximum-likelihood fit.
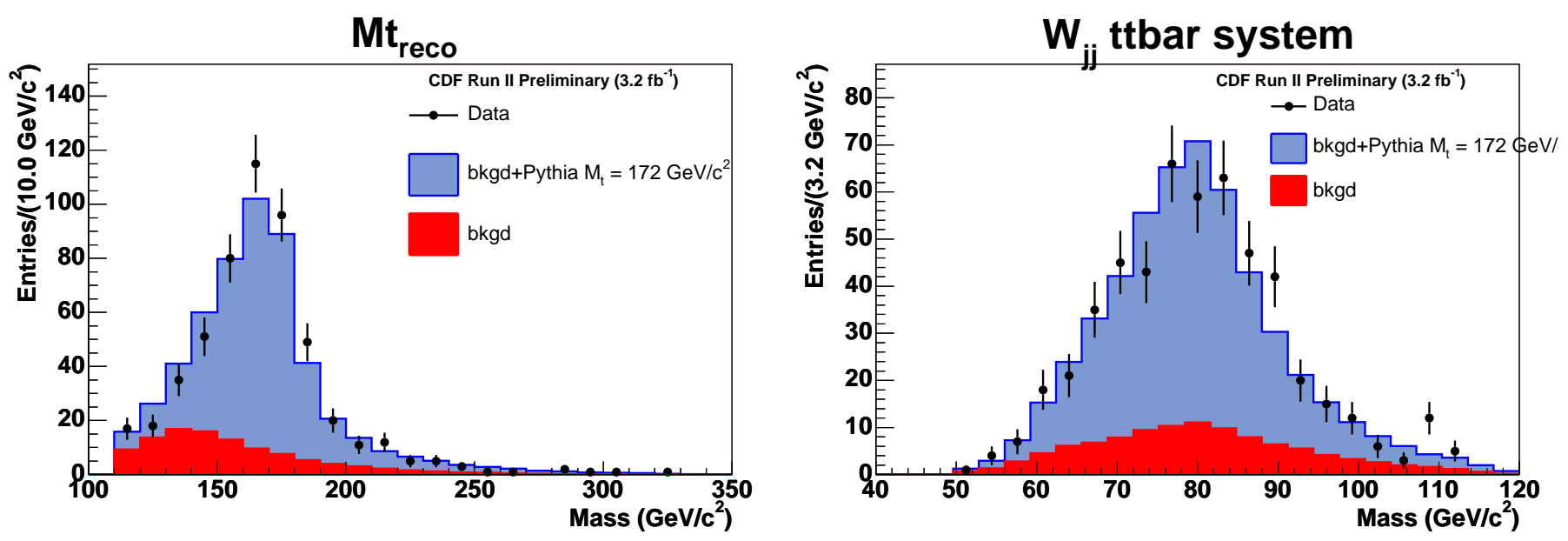

Figure 14: Top-quark mass analyses in the lepton+jets channel. The comparison between data and estimation in the lepton jet channel in the reconstructed top-quark (left) and the $W$-boson (right) mass using a template method with in situ calibration by CDF [220].

In the second method, the "Matrix Element/Dynamic Likelihood Method" (ME/DLM), similar to that originally suggested by Kondo et al. [221, 222, 223, 224] and Dalitz and Goldstein [225, 226, 227], a probability for each event is calculated as a function of $m_{t}$, using a LO matrix element for the production and decay of $t \bar{t}$ pairs. All possible assignments of reconstructed jets to final-state quarks are used, each weighted by a probability determined from the matrix element. The correspondence between measured four-vectors and parton-level four-vectors is taken into account using probabilistic transfer functions. The results of ensemble tests using the ME method at D $\varnothing$ are shown in Fig. 15,

In a third method, the "Ideogram Method" [230, 231, which combines some of the features of the above two techniques, each event is compared to the signal and background mass spectrum, weighted by the $\chi^{2}$ probability of the kinematic fit for all 24 jet-quark combinations and an event probability. The latter is determined from the signal fraction in the sample and the event-by-event purity, as determined from a topological discriminant in Monte Carlo events. An additional variation on these techniques is the "Multivariate Likelihood" (ML) technique, where an integral over the matrix element is performed for each permutation, and then summed with weights determined by the $b$-tagging information on each jet. Backgrounds are handled in the ML technique by "deweighting" events according to a background probability calculated using variables based on the topology of the event.

With at least four jets in the final state, the dominant systematic uncertainty on $m_{t}$ is from the uncertainty on the jet-energy scale. CDF (TM, ME, ML) and DØ (ME) have reduceed the jet energy scale uncertainty by performing a simultaneous, in situ fit to the $W \rightarrow j j$ hypothesis using the jets without identified secondary vertices, see Fig. 14 (right) and Fig. 16 (left). Also simultaneous measurements of the top-quark mass, the light-quark and the $b$-quark jet energy scale are proposed [232].

The fourth technique, the Decay Length Method" [233, 234] relies solely on tracking, and thus avoids the jet-energy scale uncertainty. This methods exploits the fact that, in the rest frame of the top quark, the boost given to the bottom quark has a Lorentz factor $\gamma_{b} \approx 0.4 m_{t} / m_{b}$. The measurement of the transverse decay length $L_{x y}$ of the $b$-hadrons from the top quark decay is therefore sensitive to $m_{t}$. Fig. 16 (right) displays the distribution of the two-dimensional decay length in $t \bar{t}$ candidate events at CDF. 
Dø Run Ilb Preliminary, L=2.6 fb $\mathrm{fb}^{-1}$
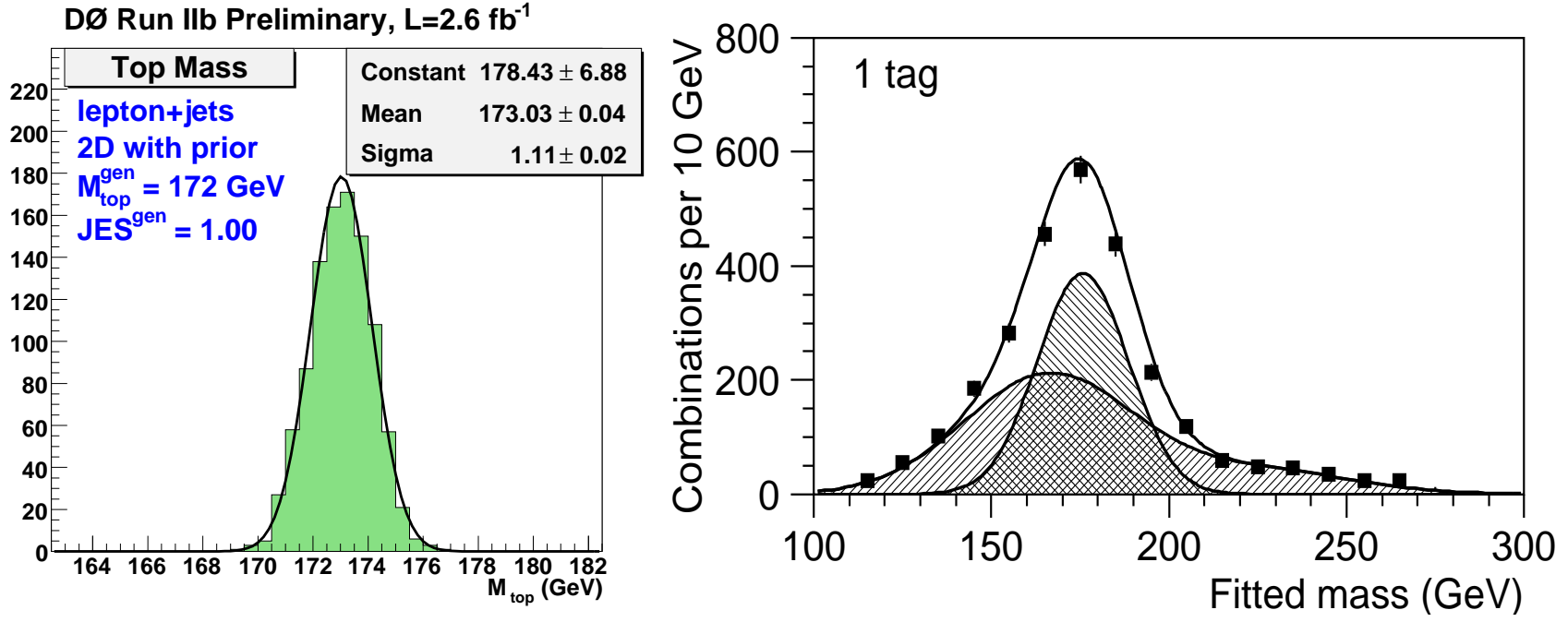

Figure 15: Top-quark mass analyses in the lepton+jets channel. Left(DØ): Distributions of fitted top quark masses from ensemble tests performed on $m_{t}^{g e n}=172.5 \mathrm{GeV} / \mathrm{c}^{2}$ and $J E S_{\text {gen }}=1.00 \mathrm{MC}$ samples using the Matrix Element Method in lepton+jets event with neural network $b$-tagging and in situ calibration is shown [228]. Right $(\mathrm{D} \varnothing)$ : Prediction of the shapes of the fitted mass distribution for the wrong and the correct permutations (hatched) and the sum of the two (black line) using the fitted parameters of an Ideogram method. The sum of the two is compared to the simulated data containing a weighted sum of all solutions (correct and wrong), for the default jet energy scale and a generated top quark mass of $175 \mathrm{GeV} / \mathrm{c}^{2}$ for events with one $b$-tag. [229]
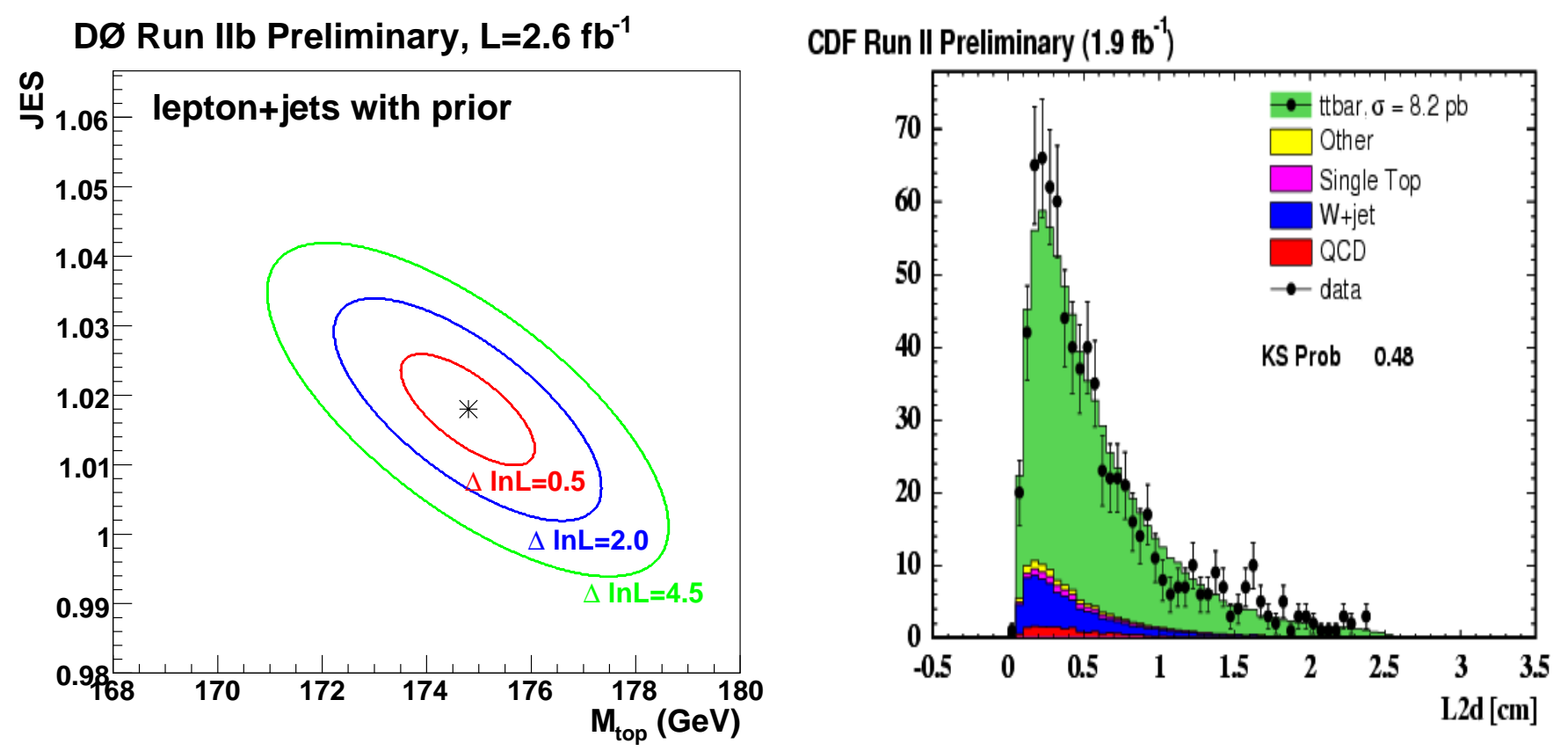

Figure 16: Top-quark mass analyses in the lepton+jets channel. Left(DØ): Calibrated result of the 2D (JES vs. $m_{t}$ ) analysis using the Matrix Element Method in lepton+jets event with neural network $b$-tagging and in situ calibration is shown [228. Right (CDF): For each lepton+jets event passing the selection, the decay length, L2d, of the two leading SecVtx tagged jets is recorded. Signal and background distributions for top mass hypotheses similar to the measured results $\left(m_{t}=178 \mathrm{GeV} / \mathrm{c}^{2}\right)$ area shown [235]. 


\subsection{Di-Lepton Channel}

Additional determinations of the top mass come from the dilepton channel with two or more jets and large missing $E_{T}$, and from the all-jets channel. The dilepton channel, with two unmeasured neutrinos, is under-constrained by one measurement. It is not possible to extract a value for the top-quark mass from direct reconstruction without adding additional information. Assuming a value for $m_{t}$, the $t \bar{t}$ system can be reconstructed up to an eight-fold ambiguity from the choice of associating leptons and quarks to jets and due to the two solutions to the $p_{z}$ of each neutrino. Recently, an analytic solution to the problem has been proposed [236]. At the Tevatron, two basic techniques are employed: one based on templates and one using matrix elements.

The first class of techniques incorporates additional information to render the kinematic system solvable. In this class, there are two techniques that assign a weight as a function of $m_{t}$ for each event based on solving for either the azimuth, $\phi$, of each neutrino given an assumed $\eta,(\boldsymbol{\eta}(\boldsymbol{\nu}))$ [237, 238, 239, or for $\eta$ of each neutrino given an assumed $\phi,(\phi(\nu))$ [240]. An alternative approach, $(\mathcal{M} W T)$ [237, 238, solves for $\eta$ of each neutrino requiring the sum of the neutrino $\vec{p}_{T}$ 's to equal the measured missing $E_{T}$ vector. In another technique, $\left(\boldsymbol{p}_{\boldsymbol{z}}(\boldsymbol{t} \overline{\boldsymbol{t}})\right)$ [241], the kinematic system is rendered solvable by the addition of the requirement that the $p_{z}$ of the $t \bar{t}$ system, equal to the sum of the $p_{z}$ of the $t$ and $\bar{t}$, be zero within a Gaussian uncertainty of $180 \mathrm{GeV} / \mathrm{c}$. In a variation of the $p_{z}(t \bar{t})$ technique, the theoretical relation between the top mass and its production cross section is used as an additional constraint. In most of the techniques in this class, a single mass per event is extracted and a top-mass value found using a Monte Carlo template fit to the single-event masses in a manner similar to that employed in the lepton+jets TM technique. The $\mathrm{D} \varnothing(\eta(\nu))$ analysis uses the shape of the weight distribution as a function of $m_{t}$ in the template fit. The $\mathbb{E}_{T}$ distribution for the events of this analysis is shown in Fig. 17 (left).

The second class, ME/DLM, uses weights based on the SM LO matrix element for an assumed mass given the measured four-vectors (and integrating over the unknowns) to form a joint likelihood as a function of $m_{t}$ for the ensemble of fitted events. Examples of this technique are shown in Fig. 17]
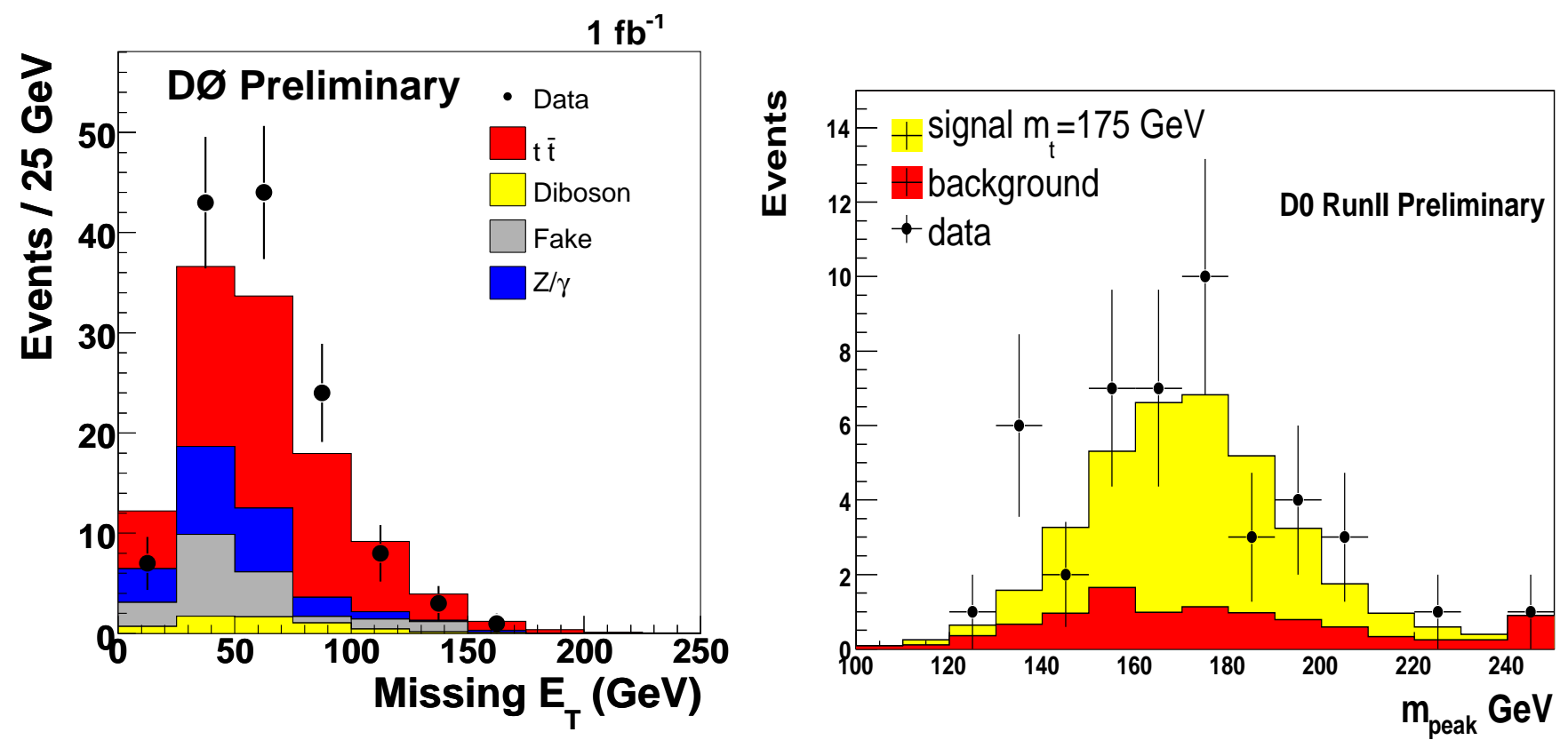

Figure 17: Top-quark mass analyses in the di-lepton channel. Left(DØ): DATA/MC comparison for the combination of dilepton channels for $E_{T}$ as used in the neutrino-weighting method with neural network $b$-tagging and a topological selection [242]. Right(DØ): Comparison of peak masses in data and Monte Carlo using a matrix weighting method with a topological selection [243]. 

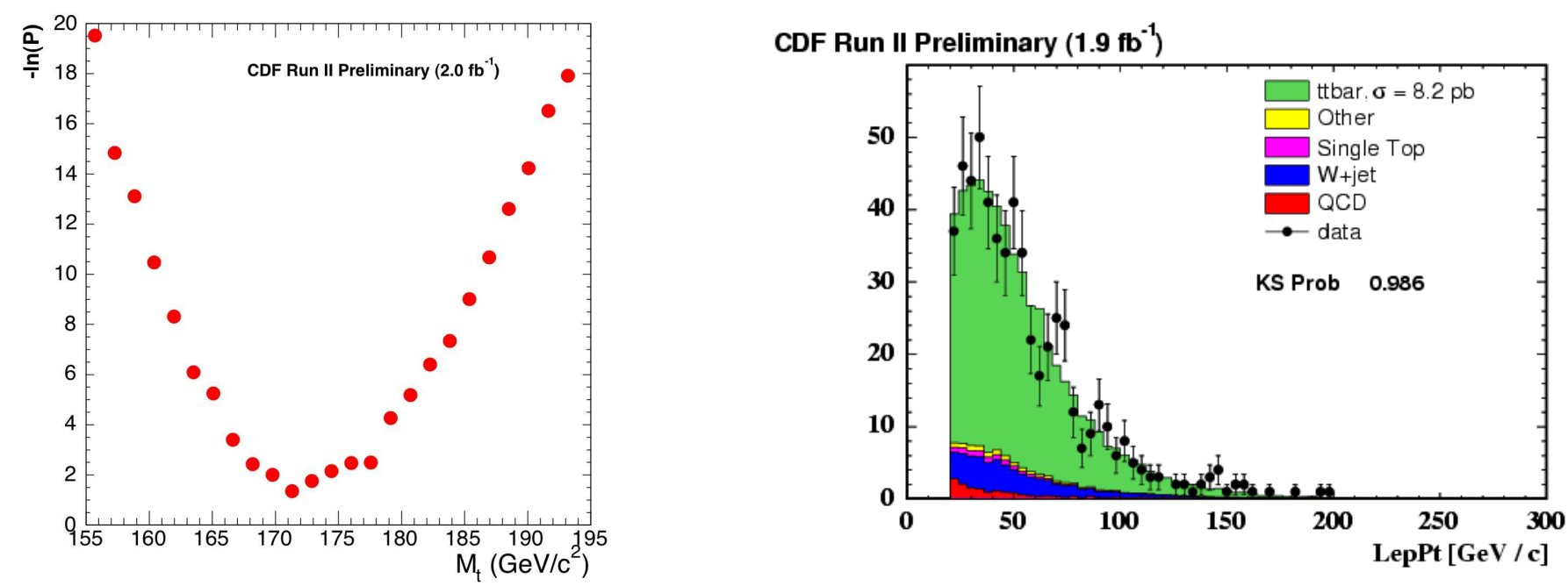

Figure 18: Top-quark mass analyses in the di-lepton channel. Left(CDF): Final posterior probability density as a function of top pole mass for the 344 candidate events in the CDF data using a matrix element method [244]. Right(CDF): Signal, background, and data for the lepton $p_{T}$ distribution, using a hypothesis top mass $m_{t}=173 \mathrm{GeV} / \mathrm{c}^{2}$ in a lepton- $p_{T}$ analysis [235].

(right) and Fig. 18 (left).

The $p_{T}$ spectrum of the leptons in the dilepton channel has also been used to extract a top mass measurement [245], see Fig. 18 (right). The resulting statistical uncertainty of the measurement is large, but as with the $L_{x y}$ technique, it is free of the systematic uncertainty due to the jet-energy scale.

In the most recent set of CDF results, a measurement has been done using the lepton+jets and dilepton channels simultaneously. In the lepton+jets channel, the TM is used together with an in situ $W \rightarrow j j$ fit. In the dilepton channel, $\eta(\nu)$ is used plus a fit to the scalar sum of transverse energies $\left(H_{T}\right)$, which is sensitive to the top mass.

\subsection{All-Jets Channel}

In the all-jets channel there is no unknown neutrino momentum to deal with, but the $\mathrm{S} / \mathrm{B}$ is the poorest. Both, CDF and D $\varnothing$, use events with 6 or more jets, of which at least one is $b$-tagged. In addition, both experiments have employed a neural network selection based on an array of kinematic variables to improve the $\mathrm{S} / \mathrm{B}$. At $\mathrm{D} \varnothing$, a top-quark mass is reconstructed from the jet-quark combination that best fits the hadronic $W$-mass constraint and the equal-mass constraint for the two top quarks. At CDF, the top-quark mass for each event was reconstructed applying the same fitting technique used in the $\ell+$ jets mode. In the most recent analysis, the in situ jet-energy scale calibration from the $W \rightarrow j j$ fit is also used. At both, CDF and DØ, the resulting mass distribution is compared to Monte Carlo templates for various top quark masses and the background distribution, see Fig. 19 for an example, and a maximum-likelihood technique is used to extract the final measured value of $m_{t}$ and its uncertainty.

$\mathrm{D} \varnothing$ also measures the top-quark mass via comparison of the $t \bar{t}$ production cross section with the Standard Model expectation [246]. This method has the advantage that it is very simple and sensitive to the top-quark pole mass, which is a very well defined concept. The fully-inclusive cross-section calculation, used for comparison, contains current best theoretical knowledge with reduced-scheme or scale-dependence.

It should be noted that the different techniques make assumptions about the SM-like production and decay of the top quark at different levels. In general, methods which make stronger assumptions about the top quark production and decay mechanism, such as the Matrix Element/Dynamic Likelihood Method, have the highest sensitivity to the top quark mass. Simple template methods in the lepton+jets 

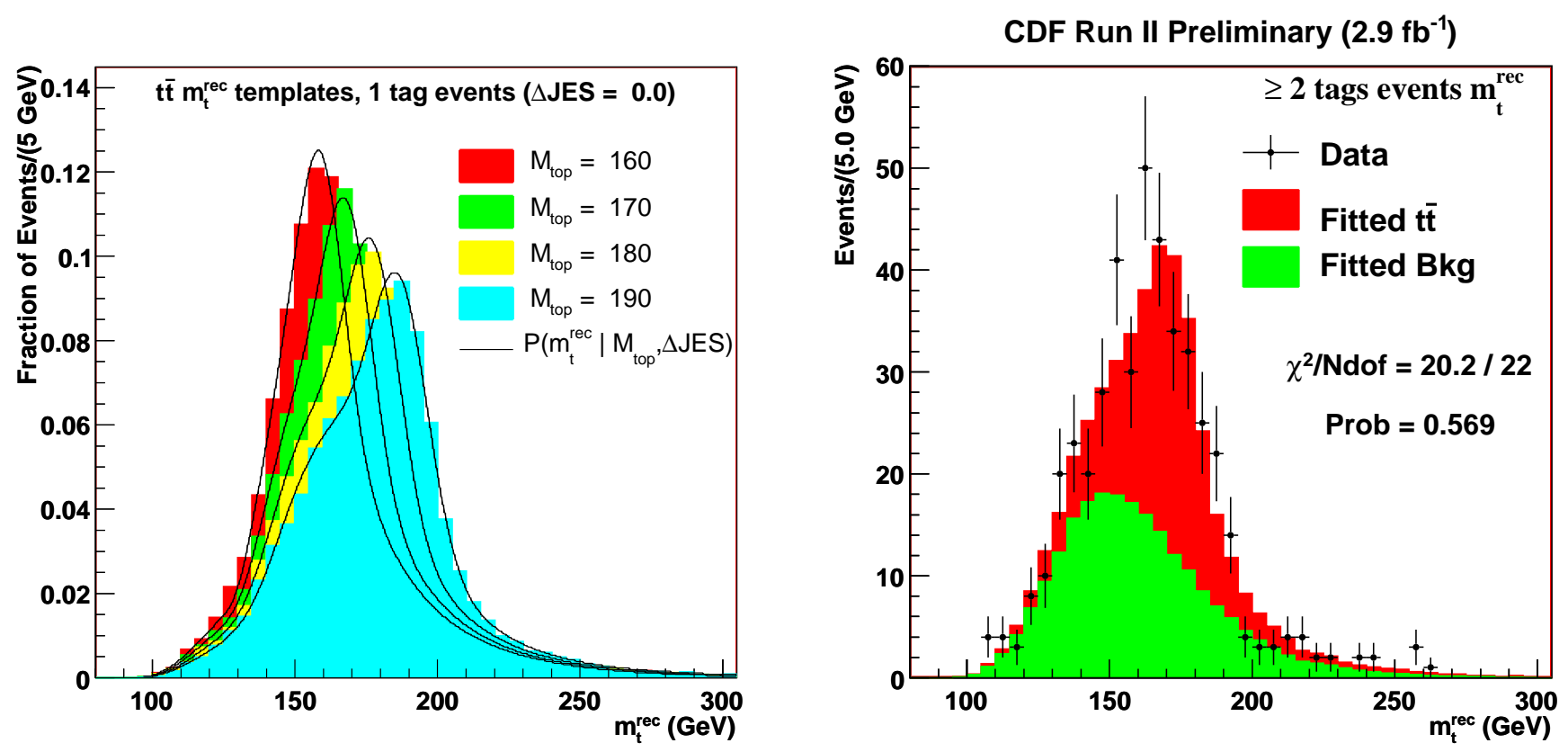

Figure 19: Top-quark mass analyses in the all-jets channel. Left(CDF): Reconstructed signal $m_{t}$ distribution, as a function of the input top mass for events with exactly $1 b$-tag, and their respective parametrization [247]. Right $(\mathrm{CDF})$ : Top reconstructed invariant masses for signal events with at least 2 b-tags [247].

channel, which only rely on energy and momentum conservation in the kinematic reconstruction and detector resolution functions, are on the one hand less sensitive to $m_{t}$, but on the other hand more stable with respect to possible modification in the details of the production and decay mechanisms involved.

The different measurements of $m_{t}$, described in this section, are summarised in Table 6. The systematic uncertainty (second uncertainty shown) is comparable to or larger than the statistical uncertainty, and is primarily due to the uncertainties in the jet-energy scale and in the Monte Carlo modeling. In the Run II analyses, CDF and D $\varnothing$ have controlled the jet-energy scale uncertainty via in situ $W \rightarrow j j$ calibration using the same $t \bar{t}$ events, as mentioned above, and via new techniques such as the decay-length technique.

The Tevatron Electroweak working Group (TevEWWG), responsible for the combined CDF/D $\varnothing$ average top mass in Table 6, took account of correlations between systematic uncertainties in the different measurements in a sophisticated manner [50]. The latest TevEWWG world average [50], including published and some preliminary Run II results, yields $m_{t}=173.1 \pm 1.3 \mathrm{GeV} / \mathrm{c}^{2}$ (statistical and systematic uncertainties combined in quadrature).

\subsection{Measurement of the Top-Quark Mass at the LHC}

At the LHC there will be 8 million $t \bar{t}$ pairs produced per year at a luminosity of $10^{33} \mathrm{~cm}^{-2} \mathrm{~s}^{-1}$. Such large event samples will permit precision measurements of the top-quark parameters. The statistical uncertainties on $m_{t}$ will become negligible, and, using essentially the methodes developed at the TEVATRON, systematic uncertainties, better than $\pm 2 \mathrm{GeV} / \mathrm{c}^{2}$ per channel are anticipated [78, 80, 79].

For example, CMs measures $m_{t}$ in the lepton+jets channel using a full kinematic fit to the events, and using the result of this fit in an event-by-event likelihood as a function of the top mass. In a data sample of $10 \mathrm{fb}^{-1}$, CMS expects a statistical uncertainty on the top-quark mass of only $200 \mathrm{MeV} / \mathrm{c}^{2}$, and a systematic uncertainty of $1.1 \mathrm{GeV} / \mathrm{c}^{2}$ if the dominating uncertainty, the $b$-jet energy scale, is known 
Table 6: Measurements of $m_{t}$ by D $\varnothing$ and CDF and their average [50]. It is a combination of Run I and Run II measurements (labelled with $\star$ ), yielding a $\chi^{2}$ of 6.3 for 10 degrees of freedom.

\begin{tabular}{|c|c|c|c|c|}
\hline$m_{t}\left(\mathrm{GeV} / \mathrm{c}^{2}\right)$ & Source & $\int \mathcal{L} d t$ & Ref. & Method \\
\hline $173.3 \pm 5.6 \pm 5.5$ & DØ Run I & 125 & [248, 249] & $\ell+$ jets, TM \\
\hline $180.1 \pm 3.6 \pm 3.9$ & DØ Run I & 125 & [250, 251] * & $\ell+$ jets, ME \\
\hline $168.4 \pm 12.3 \pm 3.6$ & DØ Run I & 125 & $238] \star$ & $\ell \ell, \eta(\nu) / \mathcal{M} W T$ \\
\hline $178.5 \pm 13.7 \pm 7.7$ & Run I & 110 & 252 & all jets \\
\hline $179.0 \pm 3.5 \pm 3.8$ & $\mathrm{D} \emptyset \quad$ Run I & $110-125$ & 250,251 & $\mathrm{D} \varnothing$ comb. \\
\hline $169.1_{-5.2}^{+5.9}$ & DØ Run II & 1000 & 253 & $\ell \ell, \ell+$ jets, $\sigma_{t \bar{t}}$ \\
\hline $173.7 \pm 0.8 \pm 1.6$ & DØ Run II & 3600 & [228] $\star$ & $\ell+$ jets $/ b$-tag, ME with $W \rightarrow j j$ \\
\hline $171.5 \pm 1.8 \pm 1.1$ & DØ Run II & 1000 & 254 & $\ell+$ jets $/ b$-tag, ME with $W \rightarrow j j$ \\
\hline $170 \pm 7$ & Run II & 1000 & 102 & $\ell+$ jets, $\sigma_{t \bar{t}}$ \\
\hline $173.7 \pm 4.4_{-2.0}^{+2.1}$ & DØ Run II & 420 & 229 & $\ell+$ jets $/ b$-tag, Ideogram \\
\hline $174.8 \pm 3.3 \pm 2.6$ & DØ Run II & 3600 & 255 * & $e \mu, \mathrm{ME}$ \\
\hline $176.0 \pm 5.3 \pm 2.0$ & DØ Run II & 1000 & 242 & $\ell \ell / b$-tag, $\eta(\nu)$ \\
\hline $175.2 \pm 6.1 \pm 3.4$ & DØ Run II & 1000 & 243 & $\ell \ell /$ topo, $\mathcal{M} W T$ \\
\hline $171.5_{-8.8}^{+9.9}$ & DØ Run II & 1000 & 256 & $\ell \ell, \tau+$ jets, $\sigma_{t \bar{t}}$ \\
\hline $174.2 \pm 0.9 \pm 1.5$ & DØ Run II & 3600 & [257] $\star$ & $\mathrm{D} \varnothing$ comb. \\
\hline $176.1 \pm 5.1 \pm 5.3$ & CDF Run I & 110 & $239,258,259] \star$ & $\ell+$ jets \\
\hline $167.4 \pm 10.3 \pm 4.8$ & CDF Run I & 110 & 239 * & $\ell \ell$ \\
\hline $186.0 \pm 10.0 \pm 5.7$ & CDF Run I & 110 & 260 * & all jets \\
\hline $176.1 \pm 6.6$ & CDF Run I & 110 & 239 & CDF comb. \\
\hline $181.3 \pm 12.4 \pm 3.5$ & CDF Run II & 2000 & 261 & $\ell+$ jets $/$ soft $-\mu$, TM \\
\hline $170.9 \pm 2.2 \pm 1.4$ & CDF Run II & 1000 & 262 & $\ell+$ jets, ME with $W \rightarrow j j$ \\
\hline $172.1 \pm 1.1 \pm 1.1$ & CDF Run II & 3200 & 263 * & $\ell+$ jets, multivariat with $W \rightarrow j j$ \\
\hline $168.9 \pm 2.2 \pm 4.2$ & CDF Run II & 1000 & 264 & $\ell+$ jets, $\mathrm{TM}$ \\
\hline $171.7_{-1.5}^{+1.4} \pm 1.1$ & CDF Run II & 3200 & 220 & $\ell \ell, \ell+$ jets, TM with $W \rightarrow j j$ \\
\hline $175.3 \pm 6.2 \pm 3.0$ & CDF Run II & 1900 & 235 * & $\ell+$ jets, Decay Length \\
\hline $172.1 \pm 7.9 \pm 3.0$ & CDF Run II & 2700 & 265 & $\ell+$ jets, Lepton $p_{T}$ \\
\hline $171.6 \pm 2.0 \pm 1.3$ & CDF Run II & 1700 & 266 & $\ell+$ jets, DLM with $W \rightarrow j j$ \\
\hline $171.2 \pm 2.7 \pm 2.9$ & CDF Run II & 1900 & 244 * & $\ell \ell, \mathrm{ME}$ \\
\hline $170.4 \pm 3.1 \pm 3.0$ & CDF Run II & 1800 & 267 & $\ell \ell, \mathrm{ME}$ \\
\hline $167.3 \pm 4.6 \pm 3.8$ & CDF Run II & 1000 & 268 & $\ell \ell / b$-tag, ME \\
\hline $172.0_{-4.9}^{+5.0} \pm 3.6$ & CDF Run II & 1800 & 269 & $\ell \ell / b$-tag, $\eta(\nu)$ \\
\hline $156 \pm 20 \pm 4.6$ & CDF Run II & 1800 & 245 & $\ell \ell$, Lepton $p_{T}$ \\
\hline $165.1_{-3.2}^{+3.3} \pm 3.1$ & CDF Run II & 2800 & 270 & $\ell \ell / b$-tag, $\phi(\nu)$ \\
\hline $169.7_{-4.9}^{+5.2} \pm 3.1$ & CDF Run II & 1200 & 271 & $\ell \ell, p_{z}(t \bar{t})$ \\
\hline $170.7_{-3.9}^{+4.2} \pm 2.6$ & CDF Run II & 1200 & 271 & $\ell \ell, p_{z}(t \bar{t})+\sigma_{t \bar{t}}$ \\
\hline $165.2 \pm 4.4 \pm 1.9$ & CDF Run II & 1900 & 272 & all jets, Ideogram \\
\hline $174.0 \pm 2.2 \pm 4.8$ & CDF Run II & 1000 & 163 & all jets, TM \\
\hline $174.8 \pm 2.4_{-1.0}^{+1.2}$ & CDF Run II & 2900 & 247] $\star$ & all jets, TM \\
\hline $171.1 \pm 3.7 \pm 2.1$ & CDF Run II & 1000 & 273 & all jets, ME+TM \\
\hline $172.6 \pm 0.9 \pm 1.2$ & CDF Run II & 3200 & 274 & CDF comb. \\
\hline $178.0 \pm 4.3$ & CDF \& D $\varnothing$ & $110-125$ & 275 & Run-I combination \\
\hline $173.1 \pm 1.3^{*}$ & $\mathrm{CDF} \& \mathrm{D} \varnothing$ & $110-340$ & 50 & world-average (2009) \\
\hline
\end{tabular}


to $1.5 \%$. In the dilepton channel already in $1 \mathrm{fb}^{-1}$ a measurement with a precision of $4.5 \mathrm{GeV} / \mathrm{c}^{2} \mathrm{can}$ be made, improving to 0.5 (stat.) \pm 1.1 (syst.) $\mathrm{GeV} / \mathrm{c}^{2}$ in $10 \mathrm{fb}^{-1}$.

Similarly, Atras studies measures the top-quark mass in the lepton+jets channel using kinematic fits. In the final expected result for $1 \mathrm{fb}^{-1}$, the systematic uncertainty dominates over the statistical one, and amounts to $0.7 \mathrm{GeV} / \mathrm{c}^{2}$ per $\%$ of $b$-jet energy scale uncertainty, $0.2 \mathrm{GeV} / \mathrm{c}^{2}$ per $\%$ of light-quark jet energy scale uncertainty, and $\sim 0.3 \mathrm{GeV} / \mathrm{c}^{2}$ due to uncertainties related to initial or final state radiation.

An interesting alternative method to measure the top mass has been studied by CMs, and involves the selection of events where a $b$-quark hadronizes into a $J / \Psi(+X)$ and the $J / \Psi$ into two leptons. The $W$-boson from the same top quark also decays leptonically. The invariant mass of the three leptons is sensitive to the top mass; the systematic uncertainties of this method include $b$-decay modelling and the lepton energy scale, but not the $b$-jet energy scale, and it is thus almost orthogonal to the standard methods. In $20 \mathrm{fb}^{-1}$ the statistical error could reach $\sim 1 \mathrm{GeV} / \mathrm{c}^{2}$ and the systematic error $\sim 1.5 \mathrm{GeV} / \mathrm{c}^{2}$, dominated by the theory systematic that may be further reduced by new calculations.

Given the experimental techniques used to extract the top mass, these mass values should be taken as representing the top pole mass. The top pole mass, like any quark mass, is defined up to an intrinsic ambiguity of order $\Lambda_{Q C D} \sim 200 \mathrm{MeV}$ [55]. For further discussion see, for example, [276] and references therein. High energy physicists around the world have started planning for a future $e^{+} e^{-}$linear collider, which may become operational in the next decade. Such a machine will offer new means for precision studies of the top quark properties and dynamics. For example, the top quark mass could be measured with a precision of $\approx 20 \mathrm{MeV} / \mathrm{c}^{2}$ from a threshold scan [63, 64, 277].

\section{Top-Quark Production and Decay Properties}

In Section 3 we have described the phenomenology of the top quark expected within the SM. To establish the top quark discovered at the TEvATRON as the SM top quark it is important to verify these properties experimentally. This section will summarize measurements of interaction properties of the top quark and the corresponding limits on possible deviations from the SM that do not assume explicit presence of non-SM particles. First measurements of the properties of electroweak interactions of the top quark shall be summarized. Then verifications of electrical properties will be described, followed by measurements of the strong interaction properties of the top quark. Finally, measurements of the top quarks width and lifetime are covered. Measurements that involve particles beyond the SM or the Higgs boson are covered in Section 9 ,

\subsection{Properties of the Electroweak Interaction of the Top Quark}

$\boldsymbol{W}$ boson helicity One of the first properties of the electroweak interaction with top quarks is that of the helicity states of the $W$ boson occurring in top-quark decays. The SM expects the top quark to decay to a $b$ quark and a $W$ boson. The $V-A$ structure of the charged weak current restricts the polarization of the $W$ boson in the SM. At the known values of $m_{t}$ and $m_{b}$ the respective fractions are about 30\% left handed (-) and about $70 \%$ longitudinal (0). Only a negligible fraction of right handed $(+) W$ bosons is expected in the SM. Depending on the $W$ helicity $(-, 0,+)$ the charged lepton in the $W$ decay prefers to align with the $b$ quark direction, stay orthogonal or escape in the direction opposite to the $b$ quark. Several observables are sensitive to these differences: the transverse momentum of the lepton, $p_{T}^{\text {lept }}$, the lepton- $b$-quark invariant mass, $M_{l b}(20$ left), and the angle between the lepton and the $b$-quark direction, $\cos \theta^{*}$. For best sensitivity at TEVATRON energies $\cos \theta^{*}$ is measured in the $W$ rest frame. 

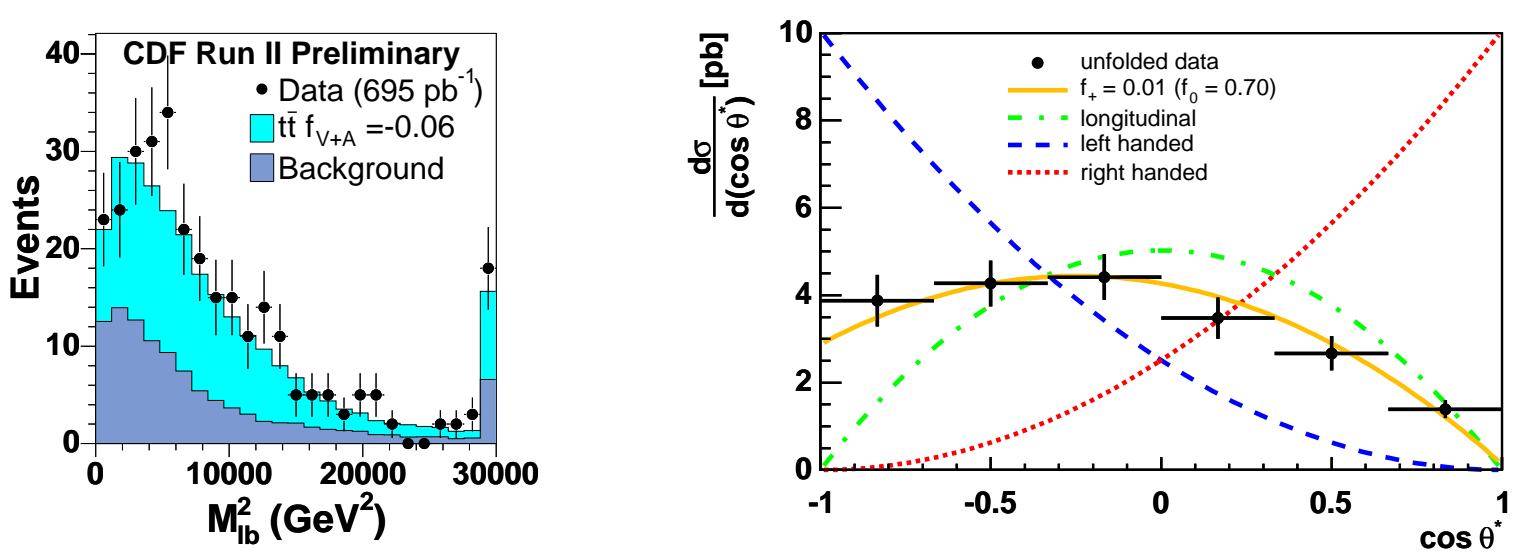

Figure 20: Distribution of the lepton- $b$-quark invariant mass squared with measured by CDF [282]. Observed $\cos \theta^{*}$ distribution after deconvolution of measurement effects, compared to expectations of the individual polarizations and the best fit combination [279].

The decay angle $\cos \theta^{*}$ has been used by both experiments in several analyses with increasing luminosity [278, 279, 280, 281]. This observable requires the reconstruction of the full top pair decay kinematics to find the rest frame of the $W$ boson that is needed to calculate $\cos \theta^{*}$. In the most recent results use datasets corresponding to luminosities between 1.9 and $2.7 \mathrm{fb}^{-1}$ [279, 281].

The CDF analyses concentrate on top-pair events in the lepton+jets channel requiring an isolated lepton, $\mathbb{E}_{T}$ and at least four jets, one of which is required to be identified as $b$-quark jet. Two methods to reconstruct the top-pair event kinematics are used. One method recovers the unmeasured neutrino momentum from the $\overrightarrow{\mathbb{E}}_{T}$ and from solving the quadratic equation that connects the neutrino and lepton momenta with $m_{W}$. The other method uses a constrained kinematic fit to determine the lepton and parton momenta, where these momenta are allowed to float within the experimental uncertainties of the measured quantities. Constraints are built from the reconstructed $W$-boson mass and the equality of the top and anti-top masses constructed from the fitted lepton and parton momenta. Both methods require the association of the measured jets to the partons of the top-pair topology and use the quality of the constrained fit to select this assignment.

The first analysis with full reconstruction uses the signal simulation to derive acceptance functions which are then convoluted with the theoretical predicted number of events in each bin of $\cos \theta^{*}$. The helicity fractions are then taken from a binned likelihood fit after subtracting the background estimation from data, see Fig. 20 (right). In the second analysis signal templates for the three different helicity states are combined the background expectations. The helicity fractions are taken from an unbinned likelihood fit with proper correction for acceptance effects. The results of both analyses agree in the analyzed $1.9 \mathrm{fb}^{-1}$ of data. They are combined with the BLUE method [283]:

$$
f_{0}=0.66 \pm 0.16(\text { stat }) \pm 0.05(\text { syst }) \quad f_{+}=-0.03 \pm 0.06(\text { stat }) \pm 0.03(\text { syst })
$$

with a correlation of about $-90 \%$ between $f_{0}$ and $f_{+}[279$.

CDF also performed an analysis using the Matrix Element technique with $1.9 \mathrm{fb}^{-1}$ and finds slightly smaller statistical uncertainties at the cost of slightly increased systematics [284].

$\mathrm{D} \varnothing$ has reconstructed the decay angle in both the lepton+jets and the dilepton channel of top pair decays. Events are selected by requiring an isolated lepton, $\mathbb{E}_{T}$ and at least four jets. No second lepton is allowed in the event. Dilepton events are selected with two isolated charged leptons with opposite charge, large $\mathbb{E}_{T}$ and at least two jets. A veto on $Z$ boson events is applied.

The top-pair decay kinematics in lepton+jets events is reconstructed using a constraint fit similar to the CDF analysis. To select the optimal jet parton assignment in addition to the fit quality, the 

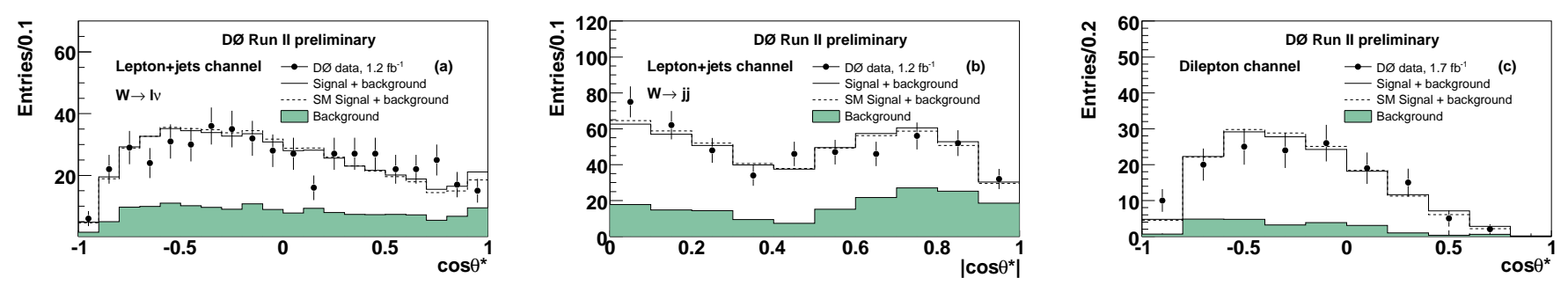

Figure 21: Distributions of $\cos \theta^{*}$ as measured in $\mathrm{D} \varnothing \mathrm{RunIIb}$ data. The left plot shows the results from the leptonic decay in lepton+jet events, the middle plot the one from the hadronic decay. The right plot shows the distribution obtained from dilepton events. In addition to the data shown with error bars all plots contain the best fit prediction is shown as full line, the SM as a dashed line histogram. The shaded area represents the background contribution [281].

probability to find the observed $b$ tags in the chosen assignment is considered. The decay angle, $\cos \theta^{*}$, is computed from the leptonic side and a second measurement of the absolute value is taken from the hadronic side. The kinematics of dilepton events can be solved assuming $m_{t}$ with a fourfold ambiguity. In addition, two possible assignments of the two leading jets in $p_{T}$ to the $b$ quarks are considered. To explore the full phase space consistent with the measurements, the measured jet and charged lepton momenta are fluctuated according to the detector resolution and $\cos \theta^{*}$ is computed for each fluctuation. The average over all solutions and all fluctuations is computed for each jet to find two $\cos \theta^{*}$ values per event. The resulting distribution for RunIIb data is shown in Fig. 21,

Simulations with specific $W$ boson helicities are used to construct a binned likelihood function, which is optimized to find the helicity fractions most consistent with the observed data. Combining the datasets of RunIIa and RunIIb to a total luminosity of $2.2-2.7 \mathrm{fb}^{-1} \mathrm{D} \varnothing$ finds

$$
f_{0}=0.490 \pm 0.106 \text { (stat) } \pm 0.085 \text { (syst) } \quad f_{+}=0.110 \pm 0.059 \text { (stat) } \pm 0.052 \text { (syst) } .
$$

The correlation between the two numbers is $-80 \%$ [281].

The lepton- $b$-quark invariant mass $M_{l b}$ has been used by CDF in lepton+jets events with one or two identified $b$-jets and and dilepton events with two identified $b$ jets. For each selected event $M_{l b}^{2}$ is computed. In lepton+jets events with a single identified $b$ jet the lepton the computation is unambiguous, though only correct in half the cases. For lepton+jets events with two identified $b$-jets a two dimensional distribution of $M_{l b}^{2}$ is constructed. One dimension being $M_{l b}^{2}$ computed with the higher energetic $b$-jet, the other with the lower energetic $b$-jet. In the dilepton events the two dimensional histogram is filled twice, i.e. using each of the leptons, c.f. Fig. 20. The distributions obtained in data are compared to simulation. Background is estimated from a combination of simulated events and data. Signal samples are simulated with left and right handed couplings. The fraction of left-handed $W$ bosons is obtained from a binned likelihood with nuisance parameters to describe the systematics: $f_{+}=-0.02 \pm 0.07$ or $f_{+}<-0.09$ at the $95 \%$ C.L. The longitudinal fraction is kept at its SM value of about 70\%. All helicity measurements are in good agreement with the SM expectations of a pure $V-A$ coupling.

The CKM element $\boldsymbol{V}_{\boldsymbol{t} \boldsymbol{b}}$ The strength of the charged weak interaction of the top quark is defined by the product of the weak coupling and the $t b$ element of the CKM matrix, $V_{t b}$. In principle this is a free parameter of the SM, but as the CKM matrix needs to be unitary, the knowledge about the other elements constrains the absolute value within the SM to $0.9990<\left|V_{t b}\right|<0.09992$ [285, 209]. Physics beyond the SM invalidates these assumptions and relaxes constraints on $\left|V_{t b}\right|$.

Deviations from the SM value modifies both the top quark decay and the weak production of single top quarks. As described in Section 6.1 the measured cross-section for single top-quark production in both experiments yields a lower limit of $\left|V_{t b}\right|>0.78$ [207, 206]. The analyses assume that top-quark decay is dominated by $t \rightarrow W b$. 

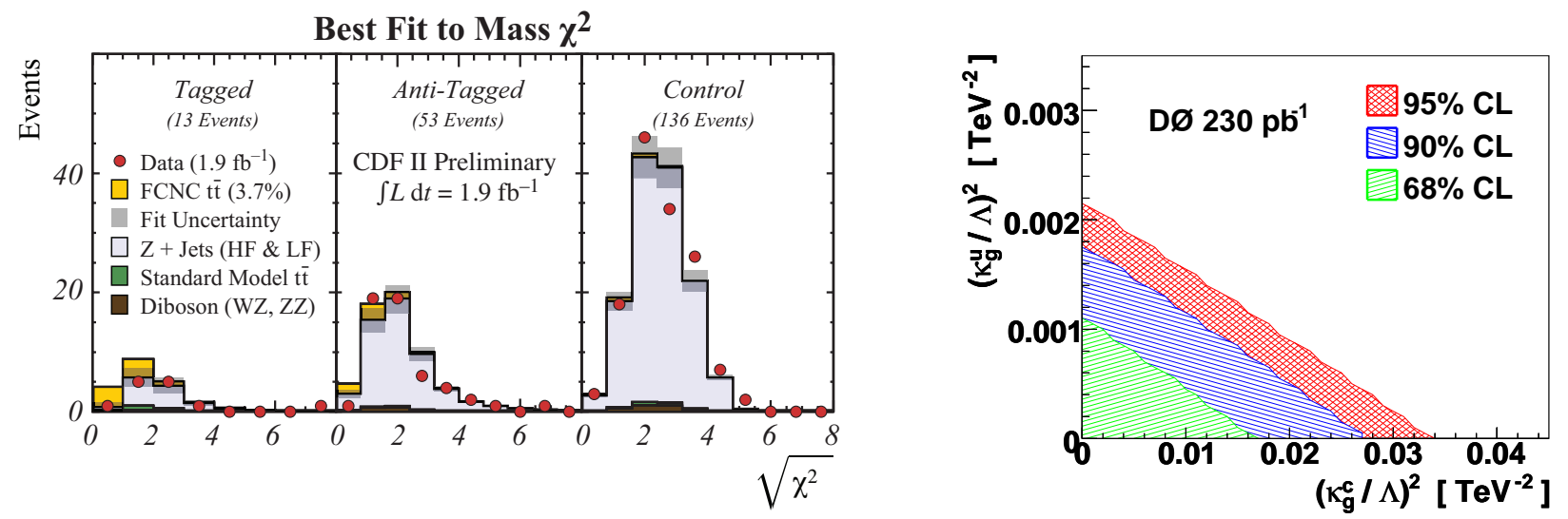

Figure 22: Left: Mass $\chi^{2}$ distribution observed by CDF in the two signal and one control sample compared the the best fit expectation plus a signal of $3.7 \%$ branching to $Z$ boson [293]. Right: Exclusion contour obtained by DØ in a two dimensional approach [291].

In top-pair events lower values of $\left|V_{t b}\right|$ reduce the amount of $b$ quarks in events with top pair signature. Both experiments have used the ratio of top-quark pair events with zero, one or two identified $b$ jets to extract the fraction of top quarks decaying to $W b: \quad R_{b}=\frac{\left|V_{t b}\right|^{2}}{\left|V_{t d}\right|^{2}+\left|V_{t s}\right|^{2}+\left|V_{t b}\right|^{2}}$. CDF has investigated lepton+jets and dilepton events and find $R_{b}$ being consistent with one [286]. DØ has investigated lepton+jets events with an integrated luminosity of $0.9 \mathrm{fb}^{-1}$. Again $R_{b}$ is found to be consistent with one and sets the currently best limit on $R_{b}>0.79$ at the $95 \%$ C.L. This limit is converted to a limit on the ratio of $\left|V_{t b}\right|^{2}$ to the off-diagonal elements: $\frac{\left|V_{t b}\right|^{2}}{\left|V_{t d}\right|^{2}+\left|V_{t s}\right|^{2}}>3.8$ at the $95 \%$ C.L. [287]. The only assumption entering this limit is that top quarks cannot decay to quarks other that the known SM quarks, which is valid even in presence of an additional generation of quarks as long as the $b^{\prime}$ quark is heavy enough. Both, the results from single top quark and those from top-quark pair events are consistent with the SM expectation.

Flavor-Changing Neutral Currents Flavor-changing neutral currents (FCNC) do not appear in the SM at tree level and are suppressed in quantum loops. Anomalous couplings could lead to enhancements of FCNC in the top sector and their observation would be a clear sign of new physics [288, 289]. The TEvatron experiments have looked for FCNC both in the (singly) production of top quarks [290, 291] and in top-quark decays [292, 293]. Limits on the single-top production through anomalous couplings were also set with LEP and HERA data [294, 295, 296, 297, 298, 299, 300].

The top decay through a $Z$ boson was searched for in $1.9 \mathrm{fb}^{-1}$ of CDF data [293. The event selection aims to identify events in which the $Z$ boson decays leptonically and the second top decay through a $W$ boson into hadrons. Two leptons of the same type and opposite charge are required with an invariant mass that lies within $15 \mathrm{GeV}$ of the $Z$ boson mass.

To separate signal from background the mass of the $W$ boson is reconstructed from two jets, the top quark is reconstructed by adding a third jet and a second top quark is reconstructed from the $Z$ boson with the fourth jet. A $\chi^{2}$ variable is built from the differences of the reconstructed masses to $m_{W}$ and $m_{t}$, respectively. The $\chi^{2}$ of the jet parton assignment that yields the lowest $\chi^{2}$ is used to build a distribution of $\chi^{2}$ values. The distribution observed in data is compared to signal and background templates derived from simulation and from control samples, c.f. Fig. 22, Data agree well with the SM expectation and thus limits on the branching fraction $t \rightarrow Z q$ are set. For this the Feldman-Cousins method is applied and yields $\mathcal{B}(t \rightarrow Z q)<3.7 \%$ at the $95 \%$ C.L. [293]. The Run I result in addition set limits on a flavor-changing currents in the photon plus jet mode of $\mathcal{B}(t \rightarrow \gamma u)+\mathcal{B}(t \rightarrow \gamma c)<3.2 \%$ [292]. 
While the above study of top-quark decays addresses FCNC through the $Z$ boson, investigations of the production of single top events can be used to restrict anomalous gluon couplings. CDF looks for the production of single top quarks without additional jets, $u(c)+g \rightarrow t$ [290]. To select such events with a leptonic top-quark decay, one isolated lepton, $\mathbb{E}_{T}$ and exactly one hadronic jet are required. The jet must be identified as $b$-quark jet. Additional cuts are used to reduce the non- $W$ backgrounds as in the single top analyses, see Section 6.1. DØ investigated a sample of single top quark production one additional jet [291]. Both experiments train neural networks to distinguish the FCNC from SM production. The neural network outputs are used to measure the cross-section of FCNC production of single top quarks. Both experiments find no evidence for such production and set limits. CDF concludes $\sigma_{t}^{\mathrm{FCNC}}<1.8 \mathrm{pb}$ at the $95 \%$ C.L. [290]. This cross-section limit is converted to limits on FCNC top-gluon coupling constants following [301, 302]. Under the conservative assumption that only one of the couplings differs from zero, CDF finds $\kappa_{\text {gtu }} / \Lambda<0.018 \mathrm{TeV}^{-1}$ or $\kappa_{\text {gtc }} / \Lambda<0.069 \mathrm{TeV}^{-1}$. Expressed in terms of the top branching fraction through these processes these limits correspond to $\mathcal{B}(t \rightarrow u+g)<3.9 \cdot 10^{-4}$ and $\mathcal{B}(t \rightarrow c+g)<5.7 \cdot 10^{-3}$ [290, 303].

$\mathrm{D} \varnothing$ computes the limits directly in terms of the anomalous gluon couplings $\kappa_{\text {gtu }} / \Lambda$ and $\kappa_{\text {gtc }} / \Lambda$. A likelihood is folded with a prior flat in the FCNC cross sections and exclusion contours are computed as contours of equal probability that contain $95 \%$ of the volume. These two-dimensional limits on the squared couplings are shown in Fig. 22 (right).

\subsection{Electrical Properties of Top Quark}

The top quark's electrical properties are fixed by its charge. However, in reconstructing top quarks the charges of the objects usually aren't checked. Thus an exotic charge value of $\left|q_{t}\right|=4 e / 3$ isn't excluded by standard analyses. To distinguish between the SM and the exotic top charge it is necessary to reconstruct the charges of the top-quark decay products, the $W$ boson and the $b$ quark.

$\mathrm{CDF}$ and $\mathrm{D} \varnothing$ have investigated lepton+jets events with at least two identified $b$ jets. CDF also includes dilepton events with one and two identified jets. The $W$ boson charge is taken from the charge of the reconstructed lepton. As a measure of the $b$ quark charge a jet charge is defined as a weighted sum of the charges of the tracks inside the $b$ jet $Q_{\text {jet }}=\sum w_{i} q_{i} / \sum w_{i}$. As a weight CDF uses the component of the track momenta longitudinal to the jet $w_{i}^{\mathrm{CDF}}=\left(\vec{p}_{i} \cdot \vec{p}_{\text {jet }}\right)^{\kappa}$ with $\kappa=0.5$; D $\varnothing$ uses the component transverse to the jet momentum $w_{i}^{\mathrm{D} \varnothing}=p_{\perp, i}^{\kappa}$ with $\kappa=0.5$. These jet charges will have significant eventto-event fluctuations, but are good for a statistical analysis. For the statistical analysis it is critical to understand the purity of the $b$-tagged jets and the amount of contributions from $c$-quark jets and from light-quark jets. Both experiments use dijet data to calibrate their jet-charge observable. Finally, one of the identified $b$ jets has to be assigned to the lepton, putting the other to hadronic top-quark decay. That assignment is taken which is best described by a kinematic fit with constraints on $m_{t}$ and $m_{W}$. For the dilepton events CDF bases the corresponding decision on the invariant mass values between the associated lepton and $b$ quark.

Likelihood methods are used to test the consistency of the data with the SM and the exotic scenario. Both experiments confirm the SM. The strongest results stems from CDF. Using data corresponding to $1.5 \mathrm{fb}^{-1}$, a Bayes factor of $2 \log B_{f}=12$ is found.

\subsection{Properties of the Strong Interaction of the Top Quark}

Forward Backward Asymmetry At the TEVATRon the initial state of proton antiproton is not an eigenstate under charge conjugation. Thus in principle also the final state may change under this operation. In QCD, however, such a charge asymmetry appears only at NLO and arises mainly from interference between contributions symmetric and anti-symmetric under the exchange of top and antitop quarks [75]. Experimentally, CDF and D0 investigated forward-backward asymmetries [304, 305, 

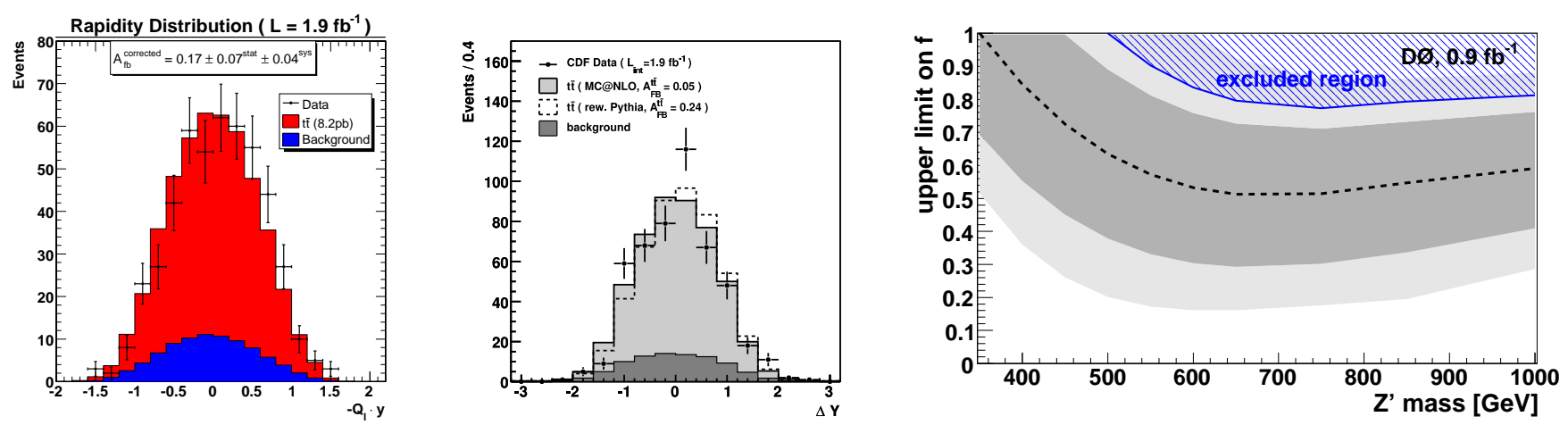

Figure 23: Distribution of the top quark rapidity (left) and rapidity difference (middle) as measured by $\mathrm{CDF}$ in $1.9 \mathrm{fb}^{-1}$ compared to the $\mathrm{SM}$ prediction [304]. Left: Limits on a possible fraction, $f$, of resonant top pair production through a $Z^{\prime}$ boson obtained from the measurement of the forward backward asymmetry in $\mathrm{D} \varnothing[306]$.

306, $A_{\mathrm{FB}}=\left(N_{\mathrm{F}}-N_{\mathrm{B}}\right) /\left(N_{\mathrm{F}}+N_{\mathrm{B}}\right)$, where $N_{\mathrm{F}}$ and $N_{\mathrm{B}}$ are the number of events observed in forward and backward direction, respectively. The forward and backward directions are either defined in the laboratory frame, i.e. according to the sign of the rapidity of the top quark, $y_{t}$, or can be defined in the frame where the top pair system rests along the beam axis, i.e., according to the sign of the rapidity difference between top and anti-top quark, $\Delta y=y_{t}-y_{\bar{t}}$. The two different definitions of forward and backward yield two different asymmetries that are labeled $A_{\mathrm{FB}}^{p \bar{p}}$ and $A_{\mathrm{FB}}^{t \bar{t}}$ according to their rest frame of definition. In the SM at NLO, asymmetries are expected to be 0.05 and 0.08 , respectively [307, but at NNLO significant corrections for the contributions from $t \bar{t}+X$ are predicted [308]. The smallness of the asymmetries expected within the SM make them a sensitive probe for new physics.

The measurements by CDF and D $\varnothing$ use lepton+jets events with at least one identified $b$ jet. For each event the kinematics is reconstructed with a kinematic fit that uses constraints on the reconstructed top quark and $W$ boson mass. The jet-to-parton assignment with the best fit is used. The rapidity of the hadronically decayed (anti-)top quark, $y_{h}$ is multiplied by the minus the charge, $Q_{\ell}$, of the lepton to obtain the top quark rapidity, $y_{t}=-Q_{\ell} y_{h}$. Correspondingly the rapidity difference $\Delta y=y_{t}-y_{\bar{t}}$ is computed as $\Delta y=Q_{\ell}\left(y_{\ell}-y_{h}\right)$,

The obtained distributions depend on the amount of background events and differ from the true shape due to acceptance and detector effects. After background subtraction CDF derives the particle level distributions inverting the acceptance efficiencies and migration probability matrices as derived from simulation with zero asymmetry using a reduced number of only four bins. The final unfolded asymmetries obtained by CDF data are [305, 304]:

$$
A_{\mathrm{FB}}^{p \bar{p}}=0.193 \pm 0.065_{\text {stat }} \pm 0.024_{\text {syst }} \quad\left(3.2 \mathrm{fb}^{-1}\right) \quad A_{\mathrm{FB}}^{t \bar{t}}=0.24 \pm 0.13_{\text {stat }} \pm 0.04_{\text {syst }} \quad\left(1.9 \mathrm{fb}^{-1}\right) .
$$

These values are larger than the 0.05 and 0.08 expected in the SM at NLO, respectively, by up to two standard deviations.

$\mathrm{D} \varnothing$ estimates and corrects for the contribution of background event using a likelihood discriminant insensitive to the asymmetry. In $0.9 \mathrm{fb}^{-1}$ of data $\mathrm{D} \varnothing$ find a final observed asymmetry [306] of

$$
A_{\mathrm{FB}}^{t \bar{t} \text { obs }}=0.12 \pm 0.08_{\text {stat }} \pm 0.01_{\text {syst }}
$$

To keep the result model independent and in contrast to the CDF results this number is not corrected for acceptance and resolution effects. Instead it needs to be compared to a theory prediction for the phase space region accepted in this analysis and corrected for dilution effects. For NLO QCD and the cuts used in this analysis $\mathrm{D} \varnothing$ evaluates $A_{\mathrm{FB}}^{t \bar{t}}=0.008$. Thus as for CDF this result corresponds to an 


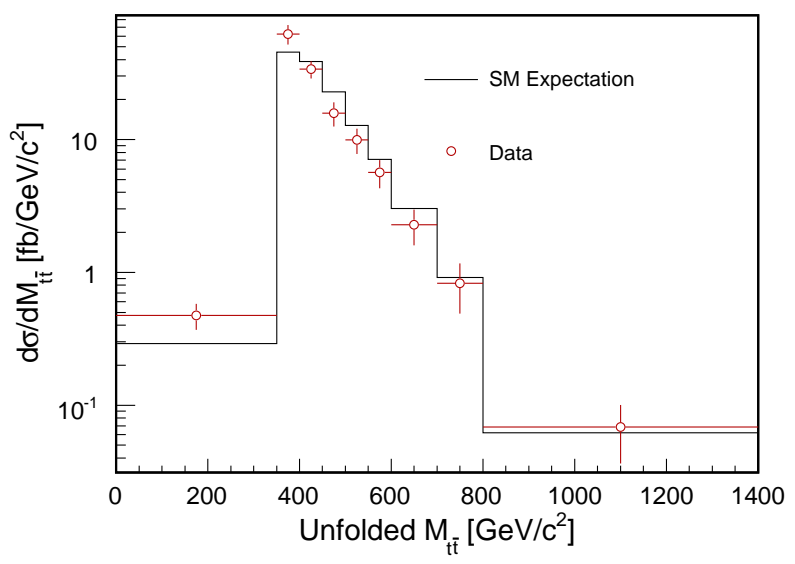

Figure 24: Left: Differential top pair production cross-section measured by CDF in $2.7 \mathrm{fb}^{-1}$ of data using the semileptonic decay mode. Indicated are the total uncertainties for each bin, excluding the overall luminosity uncertainty of $6 \%$ 309].

asymmetry that is slightly higher than expected in NLO QCD, but not by more than two standard deviations.

In addition to the QCD expectation D $\varnothing$ provides a parameterized procedure to compute the asymmetry expected in this analysis for an arbitrary model of new physics. As an example the measurement's sensitivity to top pair production via a heavy neutral boson, $Z^{\prime}$, with couplings proportional to that of the SM $Z$ boson is studied. Limits on the possible fraction of heavy $Z^{\prime}$ production are determined as function of the $Z^{\prime}$ boson mass, c.f. Fig. 23 (right). These can be applied to wide $Z^{\prime}$ resonance by averaging of the corresponding mass range.

Differential Cross-section Measurements of the differential cross-sections of top pair production can be used to verify the production mechanism assumed in the SM. Due to the required unfolding these measurements are especially cumbersome. The CDF collaboration has measured the differential cross section with respect to the invariant top pair mass $\frac{\mathrm{d} \sigma_{t \bar{t}}}{\mathrm{~d} M_{t \bar{t}}}\left(M_{t \bar{t}}\right)$ using $2.7 \mathrm{fb}^{-1}$ of lepton+jets data with one identified $b$ jet [309]. The invariant mass of the top pairs is reconstructed from the fourmomenta of the four leading jets in $p_{T}$, the four momentum of the lepton and the missing transverse energy. The $z$-component of the neutrino is not reconstructed but used as if it was zero.

The expected background is subtracted from the observed distribution and then distortions are unfolded using the singular value decomposition [310] of the response matrix that is obtained from simulations. The differential cross section obtained in $2.7 \mathrm{fb}^{-1}$ of data using the semileptonic decay mode is shown in Fig. 24. The consistency with the SM expectation has a $p$-value is 0.28 showing good agreement of with the SM. The invariant top pair mass was used in further analyses by both CDF and DØ to search for new physics. These results are described in Section 9 .

Gluon Production vs. Quark Production Production of top quark pairs in the SM occurs through strong force. At the Tevatron the dominant process is that of $q \bar{q}$ annihilation, about $15 \%$ are contributed by gluon gluon fusion. Due to large uncertainties on the gluon PDF the exact size of the gluon contribution is rather uncertain [311, 95, 100].

Two properties of the two production processes allow to separate them and to measure their relative contributions. Close to threshold the spin states of the gluon fusion are $J=0, J_{z}=0$, while the $q \bar{q}$ annihilation yields $J=1, J_{z}= \pm 1$ [312]. This yields angular correlations between the charged leptons in the dilepton channel. Alternatively one can exploit the difference in the amount of gluon 
radiation from quarks and gluons. The gluon fusion processes are expected to contain more particles from initial-state radiation.

CDF has used both features to measure the gluon fraction of top-quark pair production [313, 314. The dilepton analysis uses the azimuthal angle between the two charged leptons and compares the distribution to templates of the pure production modes. In the investigated $2.2 \mathrm{fb}^{-1}$ of dilepton events CDF obtains a gluon-fusion fraction of $0.53 \pm 0.37$ [313]. The total uncertainty is dominated by statistical uncertainties and is not yet able to restrict the theoretical uncertainties on the gluon fusion production.

The lepton+jets analysis uses the average number of of soft tracks per event, $\left\langle N_{\text {trk }}\right\rangle$, with $0.9 \mathrm{GeV}<$ $p_{T}<2.9 \mathrm{GeV}$ in the central detector region $|\eta| \leq 1.1$ as a measure of the amount of initial state radiation. This observable was found to have a linear relation with the average number of gluons in the hard process. For the measurement this relation is calibrated from samples of $W+0$ jets which have low and dijet events which have a high gluon content. In the dataset of $0.96 \mathrm{fb}^{-1} \mathrm{CDF}$ determines a gluon-fusion fraction of top-pair production in semileptonic events of $0.07 \pm 0.14_{\text {stat }} \pm 0.07_{\text {syst }}$ 314. This number corresponds to an upper limit of 0.33 at $95 \%$ C.L, well in agreement with the SM expectations. Also this measurement is statistically limited.

\subsection{Width and Lifetime}

The top-quark width $\Gamma_{t}$ and its lifetime are related by Heisenberg's uncertainty principle. In the SM $\Gamma_{t}$ is expected to be $1.34 \mathrm{GeV}$, corresponding to a very short lifetime of about $5 \cdot 10^{-25} \mathrm{~s}$. Experimentally, these predictions have been challenged for gross deviations in two different analyses. Lower limits on the lifetime are determined from the the distribution of reconstructed top mass values and upper limits on the lifetime from the distribution of lepton track impact parameters.

A limit on $\Gamma_{t}$ was obtained by CDF from the distribution of reconstructed top mass values in lepton plus jets event using $1 \mathrm{fb}^{-1}$ of data [315]. The top mass values are reconstructed in each event using a kinematic fit with constraints on the $W$ boson mass and the equality of the top and anti-top masses. The jet parton assignment which yields the best fit is used in the analysis. CDF claims that the use of the constraint of the equality of the top quark masses does not destroy the sensitivity to $\Gamma_{t}$.

To find the measured value of $\Gamma_{t}$ the distribution of top quark masses reconstructed with the best association in each event is compared to parameterized templates with varying nominal width. Templates for top pair signal events use a nominal top quark mass of $m_{t}=175 \mathrm{GeV}$. Including all systematics this CDF analysis of $1 \mathrm{fb}^{-1}$ yields an upper limit of the top quark width $\Gamma_{t}<12.7 \mathrm{GeV}$ at the $95 \%$ C.L. which corresponds to $\tau_{t}>5.2 \cdot 10^{-26} \mathrm{~s}[315]$. These limits would improve if the top quark mass used in the templates was closer to the current world average of $173.1 \mathrm{GeV}$ [50].

A limit on the top quark lifetime was obtain by CDF the lepton track impact parameter distribution in lepton plus jets events [316]. The lepton track impact parameter is computed with respect to the position of the beam line in the transverse plane at the reconstructed $z$ position of the primary vertex.

Templates of the expected distribution are derived from simulations done for an ideal detector using the resolution of the CDF detector calibrated in Drell-Yan data. The template with zero life time $c \tau_{t}=0 \mu \mathrm{m}$ describes the data best. With the Feldman-Cousins approach a limit of $c \tau_{t}<52.5 \mu \mathrm{m}$ at the $95 \%$ C.L. is set [316].

The measurements of the top quark width and lifetime are fully consistent with the SM. Only extraordinary large deviations can be excluded with these results.

\subsection{Outlook to LHC}

The measurements of the top quark interaction properties performed at the TEVATRON are in general limited by statistics. The increased cross-section at the LHC and the relatively reduced SM backgrounds help to improve the experimental constraints of top quark interaction properties. 
ATLAS has investigated the prospects for collisions of $\sqrt{s}=14 \mathrm{TeV}$ and a luminosity of $1 \mathrm{fb}^{-1}$ for various measurements [119]. For this reference scenario the expected results surpass the current TEVATRON results significantly. $W$ Helicity reaches uncertainties of 0.03 and 0.05 for $f_{+}$and $f_{0}$, respectively; top quark charge measurements can distinguish the SM from the exotic scenario by multiple standard deviations and the branching fractions of FCNC can be limited to below $10^{-2}$ and $10^{-3}$ for the $t Z q$ and the $t \gamma q$ processes, respectively. CMS has investigated a scenario of $10 \mathrm{fb}^{-1}$ and $\sqrt{s}=$ $14 \mathrm{TeV}$ [79]. At this reference point the top quark spin correlations are accessible and can be extracted with a precision of $20-30 \%$.

\section{The Top Quark as a Probe for New Physics}

The phenomenology of the top quark may also be altered by particles that are not expected within the SM. Various such particles are expected by the many models beyond the SM. They may occur in the top quark production or its decay, depending on the specific model or its parameters. Some models also contain new particles with signatures that are very similar to the SM top quark. The TEvATRON experiments have checked for all these different extensions of the SM. This section will actually start with a process that is expected in the SM though at very low rate: associated Higgs production. It will continue with new particles occurring in the top quark production, then move to beyond the SM particles in the top quark decay and finally discuss production of particles that look like the top quark but aren't.

\subsection{Top Quark Production through New Particles}

Associated Higgs Production Top pairs can also take place in association with additional particles. For parameters where Higgs bosons dominantly decay to bottom quark pairs, this associated production is a possibility to measure the Yukawa coupling. While the corresponding cross-section in the SM is too low to allow a Higgs discovery in this channel only, it still contributes to the combination of the SM Higgs searches. In models some including new physics an enhancement of $t \bar{t} H$ production is expected [317, 318, 319.

D $\varnothing$ performed an analysis searching for associated Higgs production in lepton plus jets events [320]. The analysis uses the scalar sum of transverse momenta, $H_{T}$, the number of jets and the number of jets identified as $b$-jets to discriminate the SM backgrounds and top pair production containing no Higgs from the signal.

The observed data agree with the background expectations within statistical and systematic uncertainties. Limits on $\sigma(t \bar{t} H) \cdot \mathcal{B}(H \rightarrow b \bar{b})$ are then derived using the $\mathrm{CL}_{s}$ method for Higgs masses between 105 and $155 \mathrm{GeV}$. While the limit of about 60 times the SM cross-section for $M_{H}=115 \mathrm{GeV}$ exclude unexpectedly large Higgs production in association with the top quark, its contribution to the SM Higgs search remains small.

Resonant Top Quark Pair Production Due to the fast decay of the top quark, no resonant production of top pairs is expected within the SM. However, unknown heavy resonances decaying to top pairs may add a resonant part to the SM production mechanism. Resonant production is possible for massive $Z$-like bosons in extended gauge theories [321], Kaluza-Klein states of the gluon or $Z$ boson [322, 323], axigluons [324], topcolor [172, 325], and other theories beyond the SM. Independent of the exact model, such resonant production could be visible in the reconstructed $t \bar{t}$ invariant mass.

CDF has employed several different techniques to search for resonances in the $t \bar{t}$ invariant mass distribution [326, 327, 328]. All analyses use lepton plus jets events with four or more jets. Their main difference is the method to reconstruct the $t \bar{t}$ invariant mass distribution. 


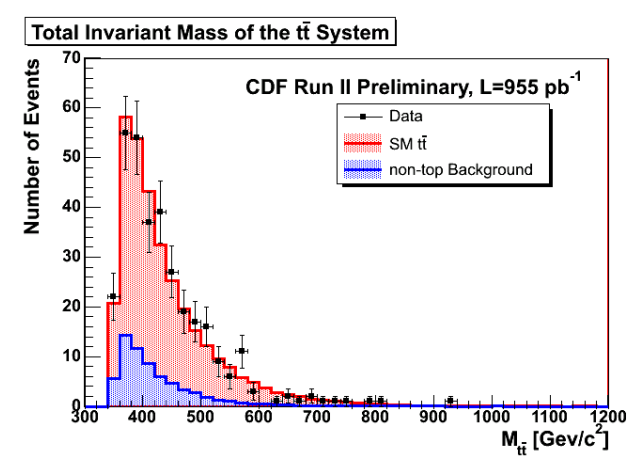

(a)

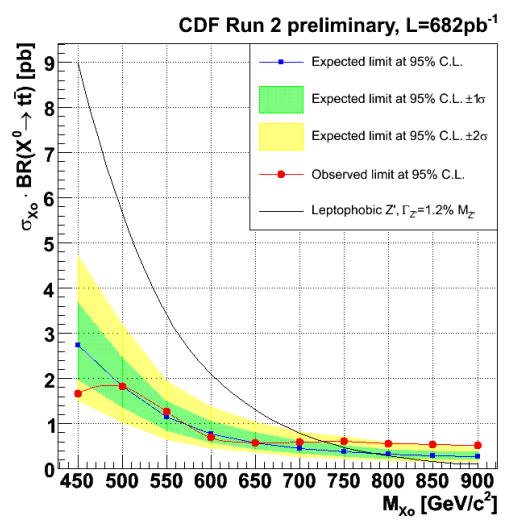

(c)

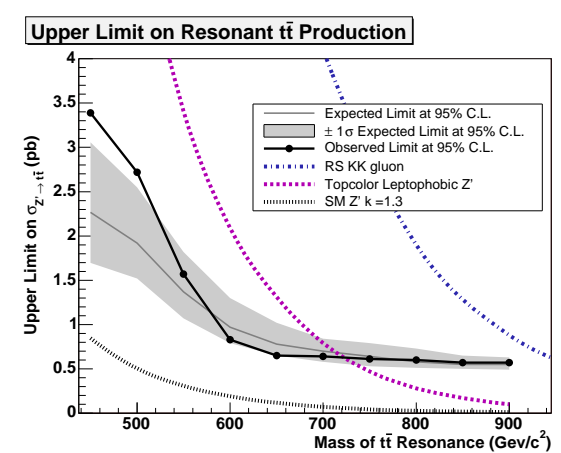

(b)

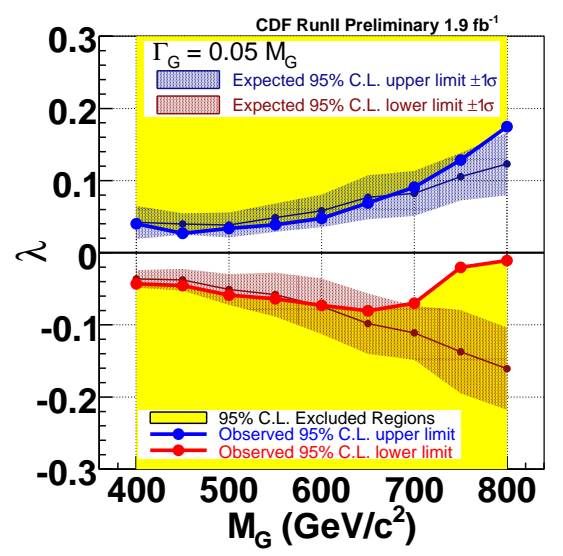

(d)

Figure 25: Distribution of the invariant top pair mass reconstructed with a constraint fit in $1 \mathrm{fb}^{-1}$ of CDF data (a) and resulting limits on the cross-section times branching fraction for resonant top pair production (b) [326]. Corresponding limits obtained the matrix-element technique in $680 \mathrm{pb}^{-1}$ of CDF data (c) 327. Theoretical curves are shown for various model and used to set mass limit on the corresponding resonance. Limits on the coupling strength of a massive gluon, $G$, contribution deduced for various masses and two widths obtained with a Matrix Element technique in $1.9 \mathrm{fb}^{-1}[\mathrm{~d})$ [328].

The method that uses the least assumptions reconstructs the invariant mass using a constraint kinematic fit [326] and is performed requiring at least one identified $b$ jet. The fit requires the reconstructed $W$ boson mass to be consistent with its nominal value and the reconstructed top quark mass to be consistent $175 \mathrm{GeV}$ within the corresponding natural widths. Of the possible jet parton assignments the one with the best fit is used to compute the top pair invariant mass for each event. The measured distribution shown in Fig. 25)(a) is compared to templates for SM production, obtained from a combination of simulation and data, and to templates of resonant production from a narrow width $Z^{\prime}$ resonance with masses between 450 and $900 \mathrm{GeV}$. The possible cross-section time branching fraction of resonant production, $\sigma_{X} \mathcal{B}(X \rightarrow t \bar{t})$, is computed from a likelihood that includes nuisance parameters to describe the systematics. Expected and observed limits are shown in Fig. 25.(b). At high resonance masses the observed limits excludes a resonant top pair production with $\sigma_{X} \mathcal{B}>0.55 \mathrm{pb}$ at $95 \%$ C.L. A leptophobic topcolor production mechanism is excluded for resonance masses up to $720 \mathrm{GeV}$.

The resolution of the reconstructed invariant top pair mass can be improved by assuming additional information. In an analysis of $680 \mathrm{pb}^{-1} \mathrm{CDF}$ employed the matrix-element technique to reconstruct the invariant mass distribution that is used to search for resonant production [327] following the mass analysis [329]. For each event a probability distribution, $P(\{\vec{p}\} \mid\{\vec{j}\})$, for the momenta of the top pair decay products (4 quarks, charged lepton and neutrino, $\{\vec{p}\}$ ) is computed given the observed quantities in that event, $\{\vec{j}\}$. This probability distribution uses parton density functions, the theoretical matrix- 
element for SM top pair production and decay and jet transfer functions that fold in the detector resolution. This probability density is then converted to a probability density for the top pair invariant mass:

$$
P_{f}\left(M_{t \bar{t}} \mid\{\vec{j}\}\right)=\int \mathrm{d}\{\vec{p}\} P(\{\vec{p}\} \mid\{\vec{j}\}) \delta\left(M_{t \bar{t}}-m(\{\vec{p}\})\right)
$$

The sum of the probabilities for all possible jet-parton assignments is used to compute the reconstructed invariant mass as mean value. Here the $b$-tagging information is used to reduce the number of allowed jet parton assignment, but the events are not required to contain $b$-tagged jets in the event selection. Again the measured distribution is compared to templates for the SM expectation and for resonant production through a narrow width $Z^{\prime}$ boson. In this analysis Bayesian statistics is employed to compute a possible contribution from such resonant production. As the data show no evidence for resonant top pair production upper limits are derived for $\sigma_{Z^{\prime}} \cdot \mathcal{B}\left(Z^{\prime} \rightarrow t \bar{t}\right)$, c.f. Fig. 25] (c), A comparison to the leptophobic topcolor assisted technicolor model yield a exclusion of this model for $M_{Z^{\prime}}<725 \mathrm{GeV}$ at 95\%C.L.

A more recent variation of this CDF analysis is used to search for a new color-octet particle, called a massive gluon, in $1.9 \mathrm{fb}^{-1}[328$. In contrast to the previously described analysis the invariant top pair mass is reconstructed without using the production part of the Matrix Element to construct the probability densities in Eq. (35) to avoid a bias towards the SM production mechanism. An unbinned likelihood fit based on the production matrix elements with and without massive gluon contribution is used to extract the possible coupling strengths of such a massive gluon contributing to the top pair production. The likelihood is computed for various the masses and widths of the massive gluon. The observed data agree with the SM expectation within $\sim 1.7 \sigma$. Limits on the possible coupling strength of a massive gluon, $G$, contributing to the top pair production are set at 95\%C.L. for various values of the width, $\Gamma_{G}$ as function of the mass, $M_{G}$. Fig. 25)(d) shows the expected and observed limits for one choice of the massive gluon width.

$\mathrm{D} \varnothing$ investigated the invariant mass distribution of top pairs in up to $3.6 \mathrm{fb}^{-1}$ of lepton plus jets events [330, 331, 332]. In these analyses the top pair invariant mass is reconstructed directly from the reconstructed physics objects. A constraint kinematic fit is not applied. Instead the momentum of the neutrino is reconstructed from the transverse missing energy which is identified with the transverse momentum of the neutrino and by solving $M_{W}^{2}=\left(p_{\ell}+p_{\nu}\right)^{2}$ for the $z$-component of the neutrino momentum. $p_{\ell}$ and $p_{\nu}$ are the four-momenta of the lepton and the neutrino, respectively. The method uses even fewer assumption first CDF analysis above. Compared to the constraint fit reconstruction it gives better performance at high resonance masses and in addition allows the inclusion of events with only three jets. Templates of the SM expectation and for resonant production through narrow width $Z^{\prime}$ are compared to data. The cross-sections of resonant production most consistent with the data are evaluated using the Bayesian technique for various resonance masses. Data agree with pure SM expectation and thus limits are set on the $\sigma_{X} \cdot \mathcal{B}(X \rightarrow t \bar{t})$ as function of the assumed resonance mass. The excluded values range from about $1 \mathrm{pb}$ for low mass resonances to less then $0.2 \mathrm{pb}$ for the highest considered resonance mass of $1 \mathrm{TeV}$. The benchmark topcolor assisted technicolor model can be excluded for resonance masses of $M_{Z^{\prime}}<820 \mathrm{GeV}$ at $95 \% \mathrm{CL}$.

Single Top Production through Charged Higgs Charged Higgs bosons appear in many extentions of the SM due to the need for an additional Higgs doublet. A charged Higgs can replace the $W$ boson in single top quark production and in top quark decays. Both effects have been searched for at the Tevatron, the latter will be described in 9.2 .

The signature for single top quark is identical to that of the SM $s$-channel, but may have a resonant structure in the invariant mass distribution of its decay products, the top and the bottom quark. Following their single top analysis, DØ selects events with an isolated lepton, missing transverse energy and exactly two jets, one of which is required to be identified as b-jet [333]. SM and charged Higgs 

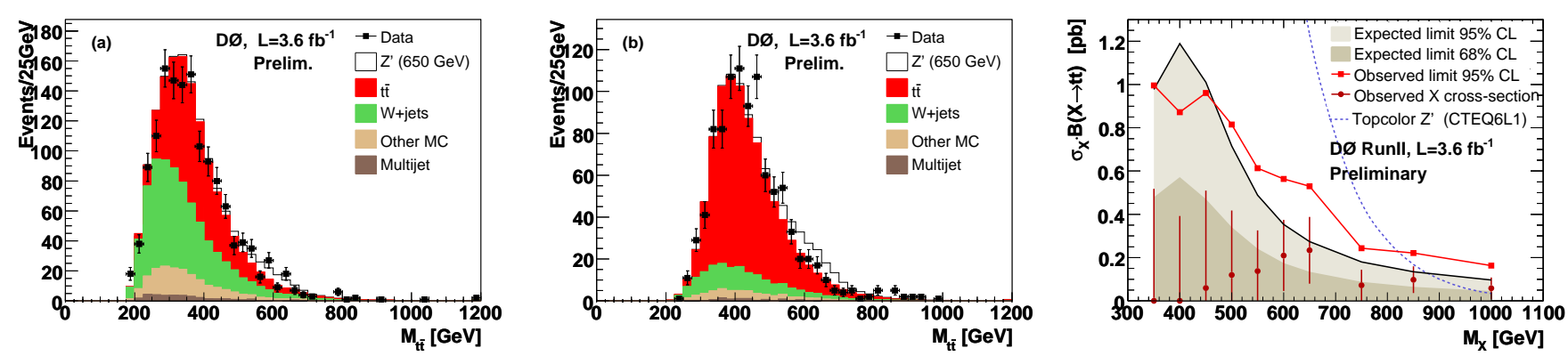

Figure 26: Expected and observed $t \bar{t}$ invariant mass distribution for the combined (left) $\ell+3$ jets and (middle) $\ell+4$ or more jets channels, with at least one identified $b$ jet. Expected upper limits on $\sigma_{X} \cdot B(X \rightarrow t \bar{t})$ (shaded) vs. the assumed resonance mass compared to the observed cross-section and exclusion limits at $95 \% \mathrm{CL}$ for $3.6 \mathrm{fb}^{-1}$ of $\mathrm{D} \varnothing$ data [332]. The prediction of the topcolor assisted technicolor model used to derive benchmark mass limits.
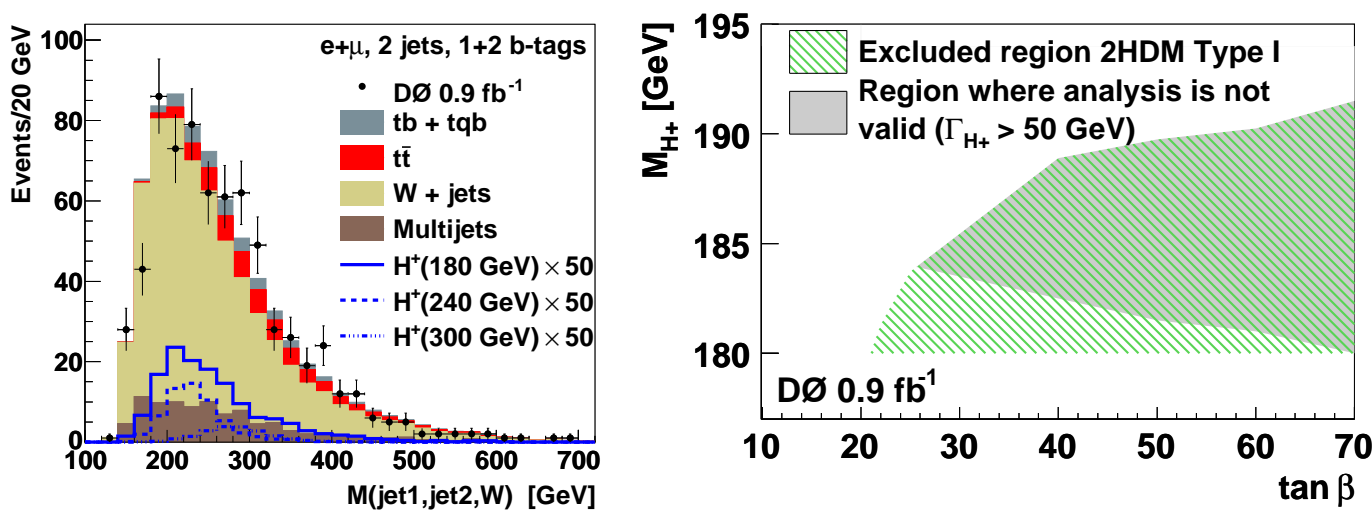

Figure 27: Left: Observed and expected invariant mass of $W$ and the two leading jets in $0.9 \mathrm{fb}^{-1}$ of $\mathrm{D} \varnothing$ Data. Right: Corresponding exclusion areas for Type I two Higgs Doublet Model (2HDM). [333.

production of single tops is separated by reconstructing the invariant mass of the two jets and the $W$ boson. This distribution shows good agreement between data and the SM expectation, see Fig. 27(left) Bayesian statistics is used to determine the allowed cross-section for single top production through a charged Higgs using templates that represent a narrow width $H^{ \pm}$boson.

Only for the Type I two Higgs doublet model, in which one Higgs gives mass to all fermions, some region in $\tan \beta$ vs. $m_{H^{ \pm}}$can be excluded, c.f. Fig. 27(right). A significant fraction of phase space is not accessible by the analysis in its current form due to the restriction to narrow $H^{ \pm}$decay widths.

Single Top Production through Heavy $\boldsymbol{W}^{\prime}$ Boson Similar to a charged Higgs boson a charged vector boson, commonly called $W^{\prime}$, may replace the SM $W$ boson in the single top quark production. The couplings of such a $W^{\prime}$ boson may be to left-handed fermions like for the SM $W$ boson or include right-handed fermions. In general a mixture of these two options is possible. If the $W^{\prime}$ boson has left handed couplings, it will have a sizeable interference with the SM $W^{ \pm}$boson [334]. For purely righthanded couplings, the leptonic decay may only occur when the right-handed neutrinos are lighter that the $W^{\prime}$ boson. In this case the decay to a top and bottom quark is an interesting channel to perform direct searches for such $W^{\prime}$ bosons.

Both CDF and D0 search for various types of $W^{\prime}$ bosons decaying to $t b$ pairs in conjunction with their single top analyses. The main discriminating observable is the reconstructed invariant mass of the decay products, which was also utilized to search for a heavy charged Higgs boson, described in the previous paragraph. 

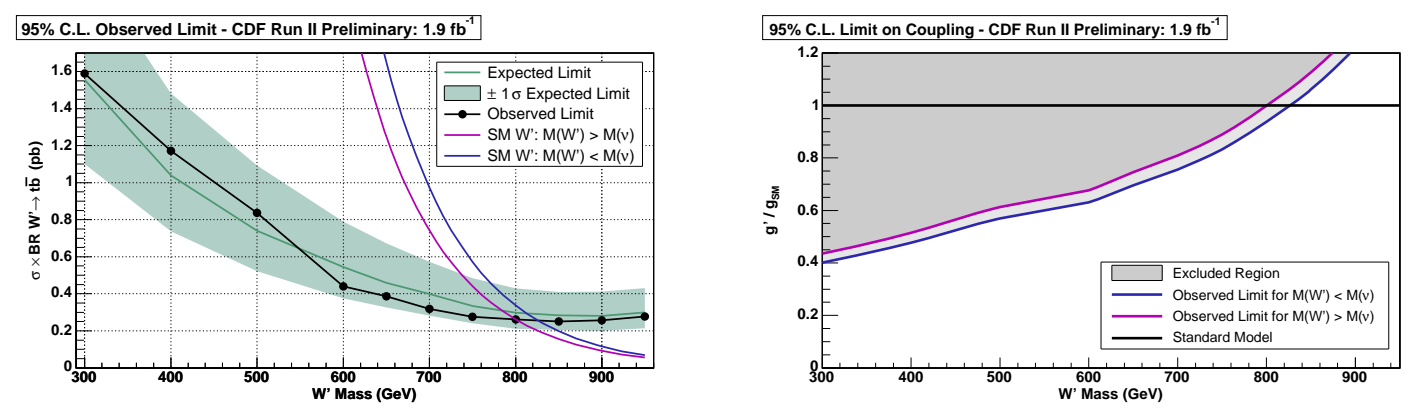

Figure 28: CDF results of a search for $W_{R}^{\prime}$ in $1.9 \mathrm{fb}^{-1}$ of data [335]. Left: Limits on $W_{R}^{\prime}$ cross-section times branching fraction to $t b$ as function $M_{W_{R}^{\prime}}$ compared to theory. Right: Limits on $W_{R}^{\prime}$ coupling strength relative the SM coupling as function $M_{W_{R}^{\prime}}$.

In an investigation of $1.9 \mathrm{fb}^{-1}$ of data 335] CDF selects $W+$ jets events requiring one lepton $(e, \mu)$ isolated from jets, missing transverse energy and two or three energetic jets. At least one of the jets must be tagged as $b$-jet. The unobserved $z$ component of the neutrino is inferred assuming the SM $W$ boson mass. The invariant mass of the lepton, the neutrino and the two leading jets, $M_{W j j}$, is then used as a discriminating observable. $W^{\prime}$ signal events are simulated for $W^{\prime}$-masses between 300 and $950 \mathrm{GeV}$ with fermion couplings identical to the $W$ boson. When the right-handed $W^{\prime}$ is heavier than the right-handed neutrinos, the branching fraction to $\ell \nu$ is corrected according to the additional decay modes. Limits on the $W_{R}^{\prime}$ boson production cross-section are set as a function of $m_{W_{R}^{\prime}}$ assuming the SM coupling strength. These are converted to mass limits by comparison to the corresponding theoretical expectation and yield $m_{W_{R}^{\prime}}>800 \mathrm{GeV}$ for $W_{R}^{\prime}$ bosons which decay leptonically and $m_{W_{R}^{\prime}}>825 \mathrm{GeV}$ for $M_{v_{R}}>M_{W_{R}^{\prime}}$. For the more general case that the $W_{R}^{\prime}$ coupling is a priori unknown the $W_{R}^{\prime}$ coupling strength, $g^{\prime}$, relative to the SM coupling, $g_{W}$, is constrained. Limits are computed from the above analysis as function of the assumed $m_{W^{\prime}}$. The observed and expected limits derived by CDF for $m_{W_{R}^{\prime}}$ and $g^{\prime} / g_{W}$ are shown in Fig. 28 .

$\mathrm{D} \varnothing$ searched for a heavy $W^{\prime}$ boson that decays to a top and a bottom quark using $0.9 \mathrm{fb}^{-1}$ of data [336]. Following their single top quark analysis events are required to have an isolated lepton, missing transverse momentum and two or three jets one of which must be identified as $b$-jet. The invariant mass of the bottom and the top decay products is computed from the measured four-momenta of the leading two jets, the charged lepton and the neutrino. Also D $\varnothing$ computes the $z$ component of the neutrino momentum assuming the SM $W$ boson mass and compares the observed distribution to templates for the SM expectation. The templates for a production through a $W^{\prime}$ boson assume the $W^{\prime}$ boson to decay to top and bottom quarks with couplings as those of the $W$ boson in the SM, though possibly to right handed fermions. D $\varnothing$ uses the Bayesian approach with a flat non-negative prior on the cross-section times branching fraction. As the data agree with the expectations of the SM, limits are derived. Expected and observed results are shown in Fig. 29, Comparing upper limits on the $W^{\prime}$ cross-section time branching fraction to top and bottom to the NLO theory predictions [337] excludes left handed $W^{\prime}$ bosons with $m_{W_{L}^{\prime}}<731 \mathrm{GeV}$. If only hadronic decays are allowed the right handed $W_{R}^{\prime}$ boson is excluded for $m_{W_{R}^{\prime}}<768 \mathrm{GeV}$, when leptonic decays are also possible the limit is $739 \mathrm{GeV}$. Without assuming the coupling strength the Bayesian approach is used to determine a limit on the size of this coupling relative to the SM, see Fig. 29 (right). Theses limits assume no interference between the SM $W$ and the $W^{\prime}$.

\subsection{Top Quark Decays including new Particles}

New particles in the final state of top pair events may alter the branching fractions to the various decay channels and modify the kinematic properties of the final state. 

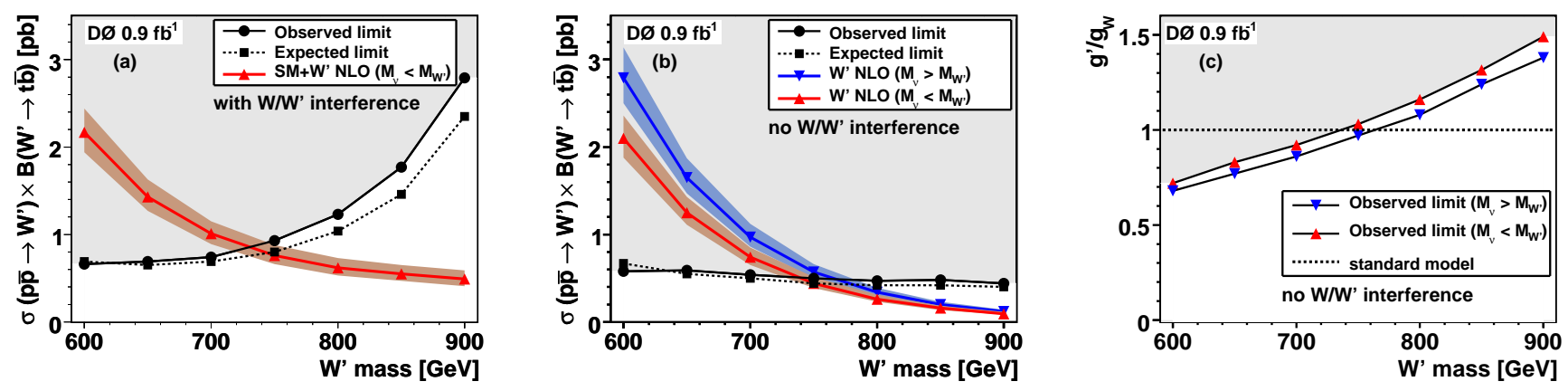

Figure 29: DØ results on a search for $W^{\prime}$ boson decaying to top and bottom using $0.9 \mathrm{fb}^{-1}$ of data. Left: Expected and observed limits on a left-handed $W^{\prime}$ production cross-section times branching fraction to top and bottom as function of $m_{W^{\prime}}$ compared to the theory prediction. Middle: Same but for right-handed $W^{\prime}$ production. Right: Limits on the $W^{\prime}$ boson coupling relative to the SM $W$-boson coupling.

Charged Higgs in Top Quark Decays An obvious candidate for such a particle is a charged Higgs boson. Because charged Higgs bosons have different branching fractions than $W$ bosons this alters the branching fractions to the various top pair decay channels. If its mass is different from the $m_{W}$ is also modifies the kinematic properties of the top pair final state. In the MSSM the decay at low $\tan \beta$ is dominated by hadronic decay to $c \bar{s}$ at low Higgs masses and to $t^{*} \bar{b}$ for Higgs masses from about $130 \mathrm{GeV}$. For $\tan \beta$ between 1 and 2 a leptonic decay to $\tau \bar{\nu}$ dominates.

A recast of the cross-section measurements performed in the $\ell+$ jets channel (with at exactly one $b$-tag or two or more $b$ tags), the dilepton channel and the $\tau+\ell$ channel has been performed by both TEVATRON experiments [338, 193]. In addition to the efficiencies for SM top pair decays, efficiencies for decays including a charged Higgs are computed.

DØ considers $H^{+} \rightarrow c \bar{s}$ and $H^{+} \rightarrow \tau^{+} \overline{\nu_{\tau}}$ decays. A likelihood for the observed number of events in the various channels is build as function of the branching fraction, $\mathcal{B}\left(t \rightarrow H^{+} b\right)$. The Feldman-Cousins procedure is used to find its allowed range. The resulting limits exclude branching fraction above around $20 \%$ for the pure leptophobic model, see Fig. 30(left). For a pure tauonic decay of the charged Higgs boson a simultaneous measurement of the top pair cross-section and the charged Higgs contribution. In such a two dimensional fit the otherwise dominating systematic uncertainty due to the assumed top pair cross-section no longer exists and the second largest uncertainty, the luminosity uncertainty, is absorbed by the fit. This results in limits that exclude charged Higgs contributions of more than $15-25 \%$ depending on the Higgs mass (Fig. 30, right).
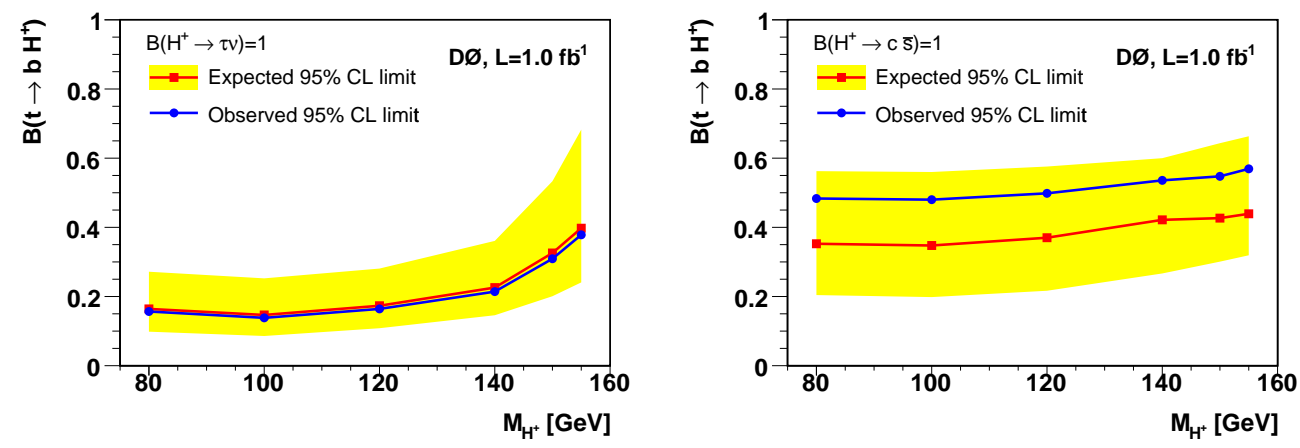

Figure 30: Limits on the contribution of charged Higgs in top decays for a leptophobic model (left) and a tauonic model (right) obtained in $1 \mathrm{fb}^{-1}$ of DØ data [193]. 


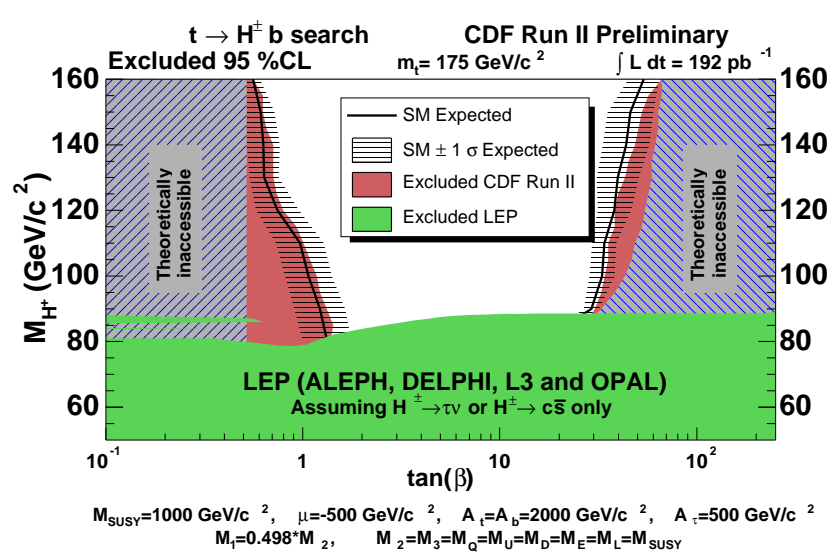

(a)

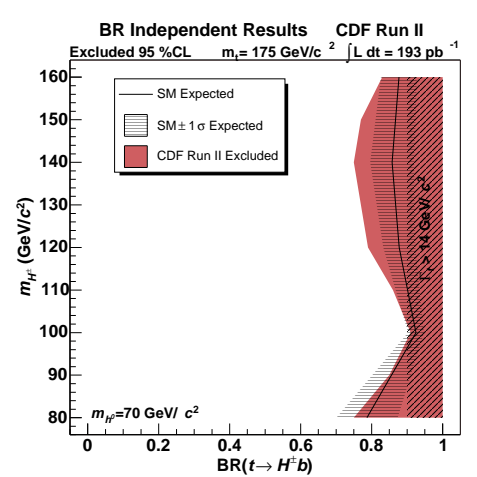

(b)

Figure 31: Results from recasting the CDF top cross-section measurements [338. (a), Exclusion region in the MSSM $m_{H^{-}} \tan \beta$-plane for an example benchmark scenario corresponding to the parameters indicated below the plot. (b): Upper limits on $\mathcal{B}\left(t \rightarrow H^{+} b\right)$ derived without assumptions on the charged Higgs branching fraction.

The CDF analysis includes the $H^{+} \rightarrow t^{*} \bar{b}$ and $H^{+} \rightarrow W^{+} h^{0} \rightarrow W^{+} b \bar{b}$ which are relevant at intermediate values of $\tan \beta$ in addition to the leptophobic and the tauonic decay. The event counts observed in data in the four channels are compared to the expectations in three different ways. For specific benchmarks of the MSSM a Bayesian approach is used set limits on $\tan \beta$. This analysis uses a flat prior on $\log \tan \beta$ within the theoretically allowed range. These limits are computed for various values of the charged Higgs mass and five different parameter benchmarks. Fig. 31](a)] shows the results for one specific benchmark.

For the high $\tan \beta$ region $H^{+} \rightarrow \bar{\tau} \nu$ dominates in a large fraction of the MSSM parameter space. Setting the branching fraction of $H^{+} \rightarrow \bar{\tau} \nu$ to $100 \%$, limits on the charged Higgs contribution to top decays are set using Baysian statistics. A flat prior for $\mathcal{B}\left(t \rightarrow H^{+} b\right)$ between 0 and 1 is used. For charged Higgs masses between $80 \mathrm{GeV}$ and $160 \mathrm{GeV}$ CDF can exclude $\mathcal{B}\left(t \rightarrow H^{+} b\right)>0.4$ at $95 \%$ C.L.

Finally, a more model independent limit is computed by scanning the full range of possible charged Higgs decay. For all five $H^{ \pm}$decay modes considered the branching fraction is scanned in steps 21 steps, assuring that the sum of branching fractions adds to one. Limits on $\mathcal{B}\left(t \rightarrow H^{+} b\right)$ are computed for each combination. The least restrictive limit is quoted. Also this analysis is repeated for various charged Higgs masses. The limits obtained in this more general approach, shown in Fig. 31)(b), exclude only very high contribution of charged Higgs to the top decay of above approx. 0.8 to 0.9 , depending on the charge Higgs mass.

At low $\tan \beta$, where the charged Higgs bosons decay also to $c \bar{s}, \mathrm{CDF}$ uses the invariant dijet mass to search for a possible contribution of a charged Higgs boson in semileptonic top pair events [339]. A kinematic fit is used to reconstruct the momenta of the top quark decay products. Constraints are employed on the consistency of the fitted lepton and neutrino momenta with the $W$ boson mass and the reconstructed top masses to be $175 \mathrm{GeV}$.

To determine a possible contribution of charged Higgs in the decay of top pair production a binned likelihood fit is performed. The likelihood is constructed with templates for signal and background with the branching fraction of top to charged Higgs, the number of top pair events and the number of background events as parameters. The observed dijet mass distribution and he fitted background composition is shown in Fig. 32)(a) including a charged Higgs contribution of 10\%. For various assumed Higgs masses 95\%CL limits on the branching fraction are determined by integrating the likelihood distribution to $95 \%$ of its total. As shown in Fig. 32(b) limits between $10 \%$ and $30 \%$ can be set 


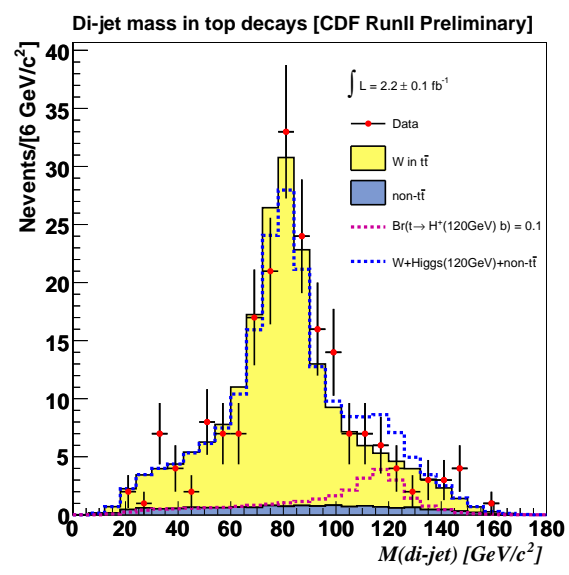

(a)

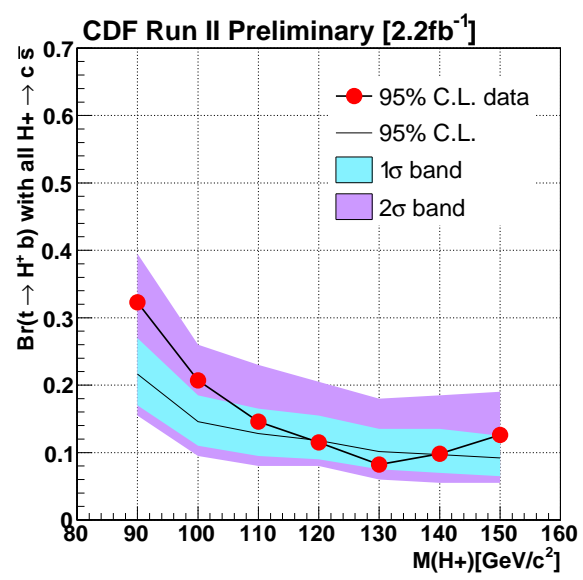

(b)

Figure 32: CDF results on search for charged Higgs using kinematic differences [339]. (a) CDF dijet mass distribution with $120 \mathrm{GeV}$ Higgs events assuming $\mathcal{B}\left(t \rightarrow H^{+} b\right)=0.1$. The size of Higgs signal corresponds to the expected upper limit branching ratio at $95 \%$ C.L. for $120 \mathrm{GeV}$. (b) CDF observed limits on $\mathcal{B}\left(t \rightarrow H^{+} b\right)$ from $2.2 \mathrm{fb}^{-1}$ data (red dots) compared with the expected limit in the SM (black line).

depending on the mass of the charged Higgs, consistent with the expected limits for pure SM top decays. This result is less model dependent than the above CDF limits, but not as strict within the models used above.

\subsection{Non-top quark signatures}

A final very fundamental question that may be asked in the context of top quark physics beyond the SM is, whether the the events that are considered to be top quarks actually are all top quarks or whether some additional unknown new particle is hiding in the selected data. CDF and D $\varnothing$ have followed this question by searching for two new particles that yield a signature similar to the SM top quark pair signature, the top quarks supersymmetric partners, stop $\tilde{t}_{1,2}$, and new heavy quarks, $t^{\prime}$.

Admixture of Stop Quarks in Top Pair Events In supersymmetry scalar partners of the left and the right handed top quarks are introduced. The lighter of the two supersymmetric partners of the top quark, the stop squark $\tilde{t}_{1}$, could be the lightest scalar quark. Within the MSSM these would be preferably produced in pairs by the strong interaction. The decay of the stop squark depends on the details of the parameters of the MSSM. Decays through neutralino and top quark, $\tilde{\chi}_{1}^{0} t$, or through chargino and $b$ quark, $\tilde{\chi}_{1}^{ \pm} b$, yield neutralino, $b$ quark and $W$ boson, $\tilde{\chi}_{1}^{0} b W$, as decay products. These have a signature very similar to that of semileptonic top quark pairs if the neutralino is the lightest supersymmetric particle and is stable due to $R$ parity conservation. In the decay through, $\tilde{\chi}_{1}^{ \pm} b$, the chargino may also result in leptonic final states, $\tilde{\chi}_{1}^{0} \ell \nu(+b)$. If this is the dominant decay stop quark pairs yield a dilepton signature.

CDF has searched for a contribution of stop quarks in the dilepton channel using upto $2.7 \mathrm{fb}^{-1}$ of data. The reconstructed stop mass is used to distinguish a stop signal from SM backgrounds including top pair production. The stop mass, $m_{\tilde{t}_{1}}$, is determined following an extention of the dilepton neutrino weighting technique. $b$-jets are assigned to their proper lepton based on jet-lepton invariant mass quantities. Neutralino and neutrino are considered as a single, though massive, pseudo particle. For given $\phi$ directions of the pseudo particles the particle momenta are determined with a fit to the measured 
Observed $95 \% \mathrm{CL}$

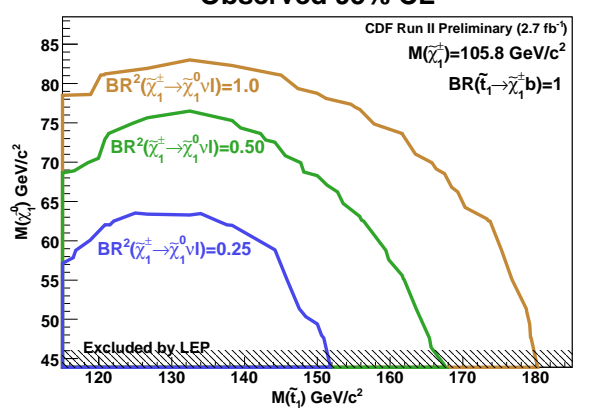

Observed $95 \% \mathrm{CL}$

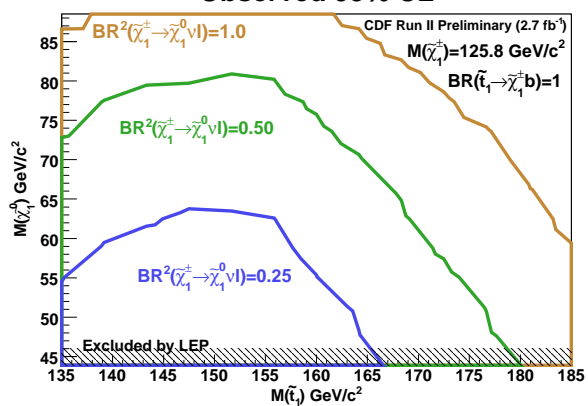

Figure 33: CDF observed 95\%CL limits in the stop mass vs. chargino mass plane. The left plot shows limits for a chargino mass of $105.8 \mathrm{GeV}$, the right for $125.8 \mathrm{GeV}$.
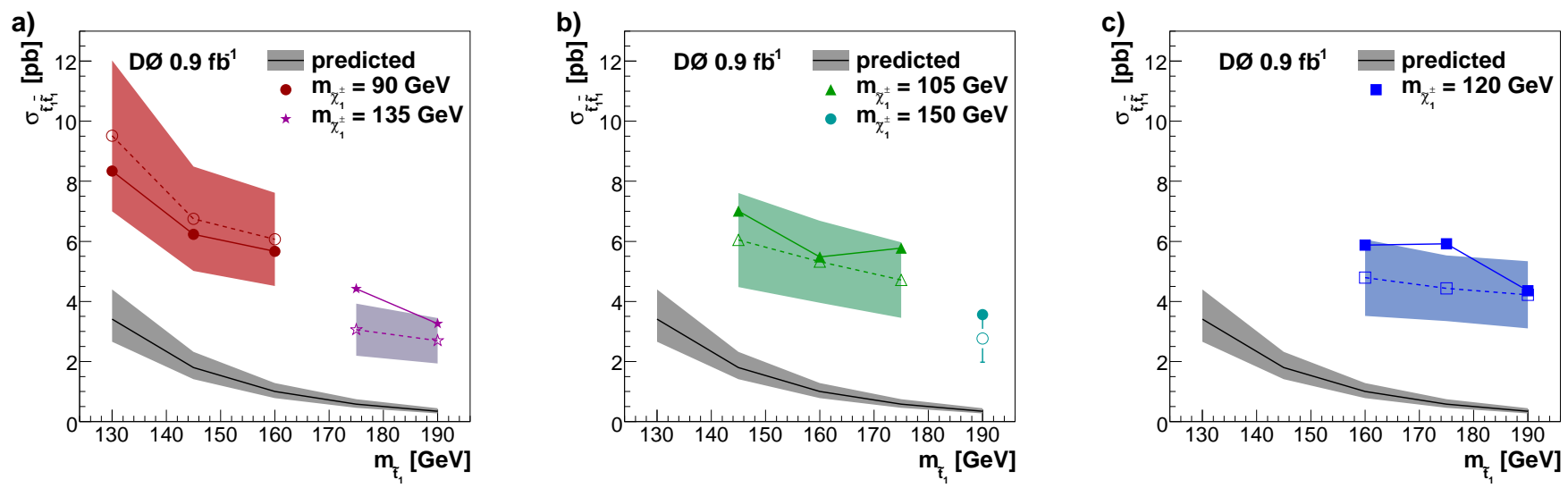

Figure 34: Expected and observed limits on the stop pair production cross-section compared to the expectation in the various MSSM parameter sets [340].

quantities using constraints on the assumed pseudo particle mass, the assumed chargino mass and the equality of the two stop masses is employed. The reconstructed stop mass is computed as weighted average of the fitted stop masses, where the average is computed over all values of $\phi$, with weights according to the fit $\chi^{2}$ probability. The combination of reconstructed stop mass templates from the signal and the various background components is fitted to data. Depending on the dilepton branching ratio limits are set in the stop vs. neutralino mass plane, c.f. Fig. 33 .

D0 has searched for a contribution of such stop pair production in the semileptonic channel in data with $\sim 0.9 \mathrm{fb}^{-1}$ [340]. The analysis uses events with at least four jets one of which is required to be identified as $b$ jet. The event kinematic is reconstructed with a kinematic fitter that assumes SM top quark pair production and applied constraints on the reconstructed $W$ boson masses and the equality on the reconstructed top quark masses. Of the possible jet parton assignments only the one with the best fit quality is considered. The differences between stop squark pair events and SM top quark pair production is assessed with a likelihood that is based on five kinematic variables. The the top quark mass reconstructed by the kinematic fitter and the invariant mass of the second and third non- $b$-tagged jet are found to have the largest individual separating power among the chosen observables.

In all cases the theoretically expected stop signal cross-section in the MSSM is much smaller than the experimental limits. Expected and observed limits on the stop pair production cross-section are derived in the Bayesian approach. The limits are compared to the MSSM prediction for various values of $m_{\tilde{t}_{1}}$ and $m_{\tilde{\chi}_{1}^{ \pm}}$in Fig. 34. That this point no limits on the MSSM parameter space can be set from this analysis. 


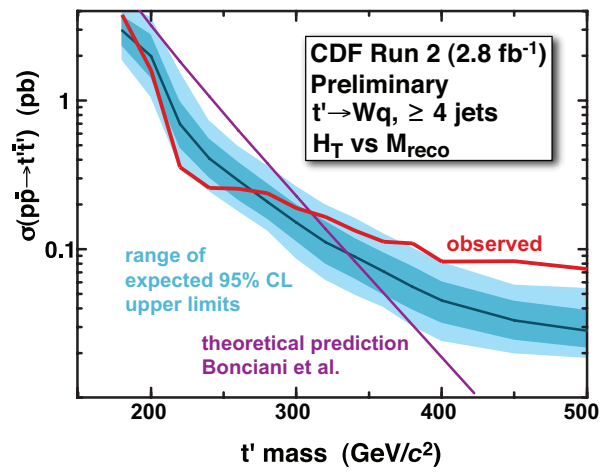

Figure 35: Expected and observed limits on the cross-section of a new top-like quark determined from $2.8 \mathrm{fb}^{-1}$ of CDF data [344]. The shaded bands show the expected one and two sigma variation on the expected upper limit.

Admixture of additional Heavy Quarks, t' Heavy top-like quarks appear in a large number of new physics models: A fourth generation of fermions [341], Little Higgs models [342] and more named in [343]. Strong bounds are placed on such models by electroweak precision data, but for special parameter choices the effects of fourth generation particles on the electroweak observables compensate each other. Among other settings a small mass splitting between the fourth $u$-type quark, $t^{\prime}$, and its isospin partner, $b^{\prime}$, is such a case, i.e. $m_{b^{\prime}}+m_{W}>m_{t^{\prime}}$ [341]. Especially, when the new top-like quarks are very heavy, they should be distinguishable from SM top production in kinematic observables. So far only CDF has performed a search for such heavy top-like quarks [343, 344]. As the $t^{\prime}$ quark decay chain is the same as the top quark decay chain a constraint fit to the kinematic properties is performed, which puts constraints on the reconstructed $W$ boson mass and the equality of the reconstructed $t^{(\prime)}$ quark masses. The heavy quark signal is distinguished from SM top quark production using two observables: the reconstructed $t^{(\prime)}$ quark mass from the kinematic fit and the scalar sum of the transverse energies of the observed jets, the lepton and the missing transverse energy, $H_{T}$. The choice explicitly avoids imposing $b$-quark tagging requirements. The signal and background shapes in the two dimensional $M_{\text {Reco }}, H_{T}$ plane are used to construct a likelihood for the observed data as function of the assumed $t^{\prime}$ cross-section, $\sigma_{t^{\prime}}$. Then Bayesian statistics is employed to compute expected and observed limits on $\sigma_{t^{\prime}}$ that are shown in Fig. 35. The limits on $t^{\prime}$ pair production cross-section, $\sigma_{t^{\prime}}$, determined in this search for a new top-like heavy quark are compared to the theoretical prediction [345]. Assuming a decay to SM quarks, $t^{\prime} \rightarrow W q$, CDF concludes that a $t^{\prime}$ pair production can be excluded for $m_{t^{\prime}}<311 \mathrm{GeV}$ at the $95 \%$ C.L. However, for masses of $m_{t^{\prime}} \simeq 450 \mathrm{GeV}$ the observed limit is worse than the expectation by more than two standard deviation, which indicates a surplus of data for that range.

\subsection{Outlook to LHC}

Also the Tevatron measurements of top-quark properties including particles beyond the SM are in general limited by statistics. The increased cross-section again helps, but not to the same amount for all processes as the increase for $q \bar{q}$ annihilation is much lower than that of gluon fusion. Many of these analyses profit even more from the increased center-of-mass energy which open additional phase space for the production of new particles.

An ATLAS study assuming collisions at $\sqrt{s}=14 \mathrm{TeV}$ and a luminosity of $1 \mathrm{fb}^{-1}$ [119] has considered the potential for discovering resonant top quark pair production through a narrow $Z^{\prime}$. A significant degradation of the selection efficiency is expected at high top pair invariant masses because the top quark decay products get joined into the same jet more and more often. At $M_{Z^{\prime}}=700 \mathrm{GeV}$ can be 


\section{Summary}

During Run II of the TEvatron top-quark physics has matured to field providing precision measurements and investigating many different aspects of the heaviest elementary particle we know today. Relatively pure sets of collision events containing a few thousand reconstructed top-quark candidates are now available for study.

The most important measurement regarding the top quark is the determination of its mass, since this parameter is essential for consistency tests of the SM and the prediction of the Higgs boson mass. When combining the TEVATRON measurements a value of $m_{t}=173.1 \pm 1.3 \mathrm{GeV} / c^{2}$ is obtained, yielding a relative precision of $0.8 \%$. The mass of the top-quark is thus the most precisely known quark mass to date.

The most challenging task in top-quark physics was the observation of single top-quark production via the weak interaction. Using advanced multivariate techniques CDF and D $\varnothing$ were able to discern this rare process from its huge background, ending a quest which had started 14 years ago with the discovery of the top quark in pair production via the strong interaction. The measurements of the single top-quark cross section indicate that the CKM matrix element $V_{t b}$ is compatible with one, the value expect in the SM.

Many other production and decay properties of the top quark have been tested using the TEVATRON data sets. The helicity of $W$ bosons in top-quark decay, the branching fractions of top quarks, and the forward-backward asymmetry in top-quark production were measured and found to be agreement with the expectations from the SM. Limits on $\Gamma_{t}$ and $\tau_{t}$ were set. Two analyses investigated the production mechansim of top quarks, supporting the SM prediction that $q \bar{q}$ annihilation dominates over $g g$ fusion.

Dedicated analyses searched top-quark data sets for phenomena beyond the SM. No evidence for right-handed couplings at the $W t b$ vertex, for FCNC in decay or production, or for heavy resonances decaying to $t \bar{t}$ pairs was found.

So far all measurements and observations in top-quark physics at the TEVATRON support the picture the SM draws of its heaviest particle. The LHC will be a top-quark factory and will allow measurements that are only limited by systematic uncertainties and may provide indications for non-SM physics. A truly encouraging perspective for a still relatively young field!

\section{Acknowledgments}

W.W. would like to thank Jan Lück from Universität Karlsruhe and Peter Uwer from Humboldt Universität Berlin for useful discussions and suggestions.

\section{References}

[1] S.J. Wimpenny and B.L. Winer, Annu. Rev. Nucl. Part. Sci. 46 (1995) 149.

[2] C. Campagnari and M. Franklin, Rev. Mod. Phys. 69 (1997) 137.

[3] P.C. Bhat, H.B. Prosper and S.S. Snyder, Int. J. Mod. Phys. A 13 (1998) 5113.

[4] K. Tollefson and E.W. Varnes, Annu. Rev. Nucl. Part. Sci. 49 (1999) 435.

[5] D. Chakraborty, J. Konigsberg and D. Rainwater, Annu. Rev. Nucl. Part. Sci. 53 (2003) 301.

[6] W. Wagner, Rept. Prog. Phys. 68 (2005) 2409.

[7] A. Quadt, Eur. Phys. J. C 48 (2006) 835. 
[8] B. Kehoe, M. Narain and A. Kumar, Int. J. Mod. Phys.A 23 (2008) 353.

[9] M.A. Pleier, Int. J. Mod. Phys. A (2008) 190.

[10] W. Bernreuther, J. Phys. G35 (2008) 083001.

[11] C. Amsler et al., Phys. Lett. B 667 (2008) 1.

[12] S.L. Glashow, Nucl. Phys. 22 (1961) 579.

[13] S. Weinberg, Phys. Rev. Lett. 19 (1967) 1264.

[14] A. Salam, ed. Nobel Symposium No. 8 (Almqvist \& Wiksell, Stockholm, 1968) .

[15] S.L. Glashow, J. Iliopoulos and L. Maiani, Phys. Rev. D 2 (1970) 1285.

[16] H. Georgi and S.L. Glashow, Phys. Rev. Lett. 28 (1972) 1494.

[17] H.D. Politzer, Phys. Rev. Lett. 30 (1973) 1346.

[18] H.D. Politzer, Phys. Rept. 14 (1974) 129.

[19] D.J. Gross and F. Wilczek, Phys. Rev. D 8 (1973) 3633.

[20] S. Weinberg, Eur. Phys. J. C 34 (2004) 5.

[21] G. 't Hooft, Nucl. Phys. B 35 (1971) 167.

[22] G. 't Hooft and M. Veltmann, Nucl. Phys. B 44 (1972) 189.

[23] G. 't Hooft and M. Veltmann, Nucl. Phys. B 50 (1972) 318.

[24] P.W. Higgs, Phys. Lett. 12 (1964) 132.

[25] F. Englert and R. Brout, Phys. Rev. Lett. 13 (1964) 321.

[26] G.S. Guralnik, C.R. Hagen and T.W.B. Kibble, Phys. Rev. Lett. 13 (1964) 585.

[27] N. Cabbibo, Phys. Rev. Lett. 10 (1963) 531.

[28] M. Kobayashi and T. Maskawa, Prog. Theor. Phys. 49 (1973) 652.

[29] S.W. Herb et al., Phys. Rev. Lett. 39 (1977) 252.

[30] G.L. Kane and M.E. Peskin, Nucl. Phys. B 195 (1982) 29.

[31] A. Bean et al., Phys. Rev. D 35 (1987) 3533.

[32] D.P. Roy and S.U. Sankar, Phys. Lett. B 243 (1990) 296.

[33] H. Albrecht et al., Phys. Lett. B 192 (1987) 245.

[34] H. Albrecht et al., Phys. Lett. B 324 (1994) 249.

[35] J. Bartelt et al., Phys. Rev. Lett. 71 (1993) 1680.

[36] S. Schael et al., Phys. Rept. 427 (2006) 257.

[37] C. Berger et al., Phys. Lett. B 76 (1978) 243.

[38] J.K. Bienlein et al., Phys. Lett. B 78 (1978) 360.

[39] W.E. Darden et al., Phys. Lett. B 76 (1978) 246.

[40] A. Shimonaka et al., Phys. Lett. B 268 (1991) 457.

[41] E. Elsen et al., Z. Phys. C 46 (1990) 349.

[42] H.J. Behrend et al., Z. Phys. C 47 (1990) 333.

[43] D. Schaile and P.M. Zerwas, Phys. Rev. D 45 (1992) 3262.

[44] J.Z. Bai et al., Phys. Rev. Lett. 88 (2002) 101802.

[45] T. van Ritbergen and R.G. Stuart, Phys. Rev. Lett. 82 (1999) 488.

[46] F. Abe et al., Phys. Rev. Lett. 74 (1995) 2626.

[47] S. Abachi et al., Phys. Rev. Lett. 74 (1995) 2632.

[48] S. Schael et al., CERN-PH-EP/2008-020 (2008), hep-ex/08114682. 
[49]. The LEP Electroweak Working Group, Updated for 2009 winter conferences: http://www.cern.ch/LEPEWWG, 2009 .

[50] T. Aaltonen et al., arXiv: 0903.2503 [hep-ex] (2009).

[51] R. Barate et al., Phys. Lett. B 565 (2003) 61.

[52] T. Aaltonen et al., arXiv: 0903.4001 [hep-ex] (2009).

[53] G.P. Zeller et al., Phys. Rev. Lett. 88 (2002) 091802.

[54] C. Quigg, Phys. Today 50N5 (1997) 20.

[55] M. Smith and S. Willenbrock, Phys. Rev. Lett. 79 (1997) 3825.

[56] M. Martinez and R. Miquel, Eur. Phys. J. C 27 (2003) 49.

[57] A.H. Hoang et al., Eur. Phys. J. direct C 2 (2000) 1.

[58] A.H. Hoang et al., Phys. Rev. Lett. 86 (2001) 1951.

[59] D. Chang, W.F. Chang and E. Ma, Phys. Rev. D 59 (1999) 091503.

[60] D. Chang, W.F. Chang and E. Ma, Phys. Rev. D 61 (2000) 037301.

[61] D. Choudhury, T.M.P. Tait and C.E.M. Wagner, Phys. Rev. D 65 (2002) 053002.

[62] U. Baur, M. Buice and L.H. Orr, Phys. Rev. D 64 (2001) 094019.

[63] J.A. Aguilar-Saavedra et al., SLAC-REPRINT-2001-002, DESY-2001-011, ECFA-2001-209, 2001 (unpublished), hep-ph/0106315.

[64] T. Abe et al., SLAC-570, 2001 (unpublished), hep-ex/0106055, hep-ex/0106056, hep-ex/0106057, hep-ex/0106058.

[65] I.I.Y. Bigi et al., Phys. Lett. B 181 (1986) 157.

[66] J.H. Kühn, Nucl. Phys. B 237 (1984) 77.

[67] A. Czarnecki, M. Jeżabek and J.H. Kühn, Nucl. Phys. B 351 (1991) 70.

[68] W. Bernreuther and A. Brandenburg, Phys. Rev. D 49 (1994) 4481.

[69] W. Bernreuther, A. Brandenburg and P. Uwer, Phys. Lett. B 368 (1996) 153.

[70] W.G.D. Dharmaratna and G.R. Goldstein, Phys. Rev. D 53 (1996) 1073.

[71] G. Mahlon and S. Parke, Phys. Rev. D 53 (1996) 4886.

[72] T. Stelzer and S. Willenbrock, Phys. Lett. B 374 (1996) 169.

[73] W. Bernreuther, M. Fücker and Z.G. Si, Phys. Lett. B 633 (2006) 54.

[74] J.H. Kühn and G. Rodrigo, Phys. Rev. Lett. 81 (1998) 49.

[75] J.H. Kühn and G. Rodrigo, Phys. Rev. D 59 (1999) 054017.

[76] M.T. Bowen, S.D. Ellis and D. Rainwater, Phys. Rev. D 73 (2006) 014008.

[77] S. Willenbrock, Lectures presented at the 12th Advanced Study Institute on Techniques and Concepts of High Energy Physics, St. Croix, U.S. Virgin Islands, 2002 , hep-ph/0211067.

[78] . ATLAS, CERN-LHCC-99-15 (1999).

[79] G.L. Bayatian et al., J. Phys. G 34 (2007) 995.

[80] G. Aad et al., CERN-OPEN-2008-20 (2008) 1.

[81] A. Bodek et al., Phys. Rev. D 20 (1979) 1471.

[82] C. Adloff et al., Nucl. Phys. B 497 (1997) 3.

[83] S. Chekanov et al., Eur. Phys. J. C 42 (2005) 1.

[84] J. Pumplin et al., JHEP 07 (2002) 012.

[85] A.D. Martin et al., Phys. Lett. B 652 (2007) 292.

[86] S. Alekhin, K. Melnikov and F. Petriello, Phys. Rev. D 74 (2006) 054033. 
[87] J. Blumlein, H. Bottcher and A. Guffanti, Nucl. Phys. B 774 (2007) 182.

[88] S. Moch and P. Uwer, Phys. Rev. D 78 (2008) 034003.

[89] V.D. Barger and R.J.N. Phillips, Collider Physics,Frontiers in Physics (Addison-Wesley, Reading, 1987).

[90] P. Nason, S. Dawson and R.K. Ellis, Nucl. Phys. B 303 (1988) 607.

[91] W. Beenakker et al., Phys. Rev. D 40 (1989) 54.

[92] W.A. Bardeen et al., Phys. Rev. D 18 (1978) 3998.

[93] W. Bernreuther et al., Phys. Rev. Lett. 87 (2001) 242002.

[94] M. Czakon and A. Mitov, (2008).

[95] M. Cacciari et al., JHEP 0404 (2004) 068.

[96] E. Laenen, J. Smith and W.L. van Neerven, Phys. Lett. B 321 (1994) 254.

[97] E. Laenen, J. Smith and W.L. van Neerven, Nucl. Phys. B 369 (1992) 543.

[98] M. Cacciari et al., JHEP 09 (2008) 127.

[99] N. Kidonakis and R. Vogt, Phys. Rev. D 78 (2008) 074005.

[100] S. Moch and P. Uwer, Nucl. Phys. Proc. Suppl. 183 (2008) 75.

[101] T. Aaltonen et al., CDF conf. note 9448, 2008.

[102] V.M. Abazov et al., Phys. Rev. Lett. 100 (2008) 192004.

[103] A.P. Heinson, A.S. Belyaev and E.E. Boos, Phys. Rev. D 56 (1997) 3114.

[104] S.S.D. Willenbrock and D.A. Dicus, Phys. Rev. D 34 (1986) 155.

[105] S. Dawson and S.S.D. Willenbrock, Nucl. Phys. B 284 (1987) 449.

[106] C.P. Yuan, Phys. Rev. D 41 (1990) 42.

[107] T. Stelzer, Z. Sullivan and S.S.D. Willenbrock, Phys. Rev. D 56 (1997) 5919.

[108] B.W. Harris et al., Phys. Rev. D 66 (2002) 054024.

[109] Z. Sullivan, Phys. Rev. D 70 (2004) 114012.

[110] J. Campbell, R.K. Ellis and F. Tramontano, Phys. Rev. D 70 (2004) 094012.

[111] N. Kidonakis, Phys. Rev. D 74 (2006) 114012.

[112] N. Kidonakis, Phys. Rev. D 75 (2007) 071501.

[113] D. Carlson and C.P. Yuan, Phys. Lett. B 306 (1993) 386.

[114] G. Mahlon and S. Parke, Phys. Rev. D 55 (1997) 7249.

[115] T. Stelzer, Z. Sullivan and S.S.D. Willenbrock, Phys. Rev. D 58 (1998) 094021.

[116] M. Jezabek, Nucl. Phys. Proc. Suppl. 37B (1994) 197.

[117] T.M.P. Tait, Phys. Rev. D 61 (1999) 34001.

[118] A. Belyaev and E. Boos, Phys. Rev. D 63 (2001) 034012.

[119] G. Aad et al., arXiv: 0901.0512 [hep-ex] (2009).

[120] J.H. Kühn, arXiv: hep-ph/9707321 (1997).

[121] M. Jezabek and J.H. Kühn, Nucl. Phys. B 314 (1989) 1.

[122] M. Jezabek and J.H. Kühn, Phys. Lett. B 207 (1988) 91.

[123] S. Mrenna and C.P. Yuan, Phys. Rev. D 46 (1992) 1007.

[124] A. Denner and T. Sack, Nucl. Phys. B 358 (1991) 46.

[125] R. Migneron et al., Phys. Rev. Lett. 66 (1991) 3105. 
[126] M. Jezabek and J.H. Kühn, Phys. Rev. D 48 (1993) R1910, Erratum: Phys. Rev. D 49 (1994) 4970.

[127] J.H. Kühn, Act. Phys. Pol. B 12 (1981) 347.

[128] M. Fischer et al., Phys. Rev. D 63 (2001) 031501.

[129] M. Fischer et al., Phys. Rev. D 65 (2002) 054036.

[130] H.S. Do et al., Phys. Rev. D 67 (2003) 091501.

[131] M. Jezabek and J.H. Kühn, Phys. Lett. B 329 (1994) 317.

[132] G. Mahlon, arXiv: hep-ph/9811219 (1998).

[133] M. Jacob and K. Johnsen, Invited talk given at last Meeting of the ISR Committee, Geneva, Switzerland, Jan 27, 1984.

[134] G. Arnison et al., Phys. Lett. B122 (1983) 103.

[135] M. Banner et al., Phys. Lett. B122 (1983) 476.

[136] T. Aaltonen et al., arXiv: 0808.0147 [hep-ex] (2008).

[137] J.R. Incandela, Nucl. Instrum. Meth. A 579 (2007) 712.

[138] D.E. Amidei et al., Nucl. Instrum. Meth. A 350 (1994) 73.

[139] S. Cihangir et al., Nucl. Instrum. Meth. A 360 (1995) 137.

[140] R. Blair et al., FERMILAB-Pub-96/390-E (1996).

[141] A. Sill et al., Nucl. Instr. and Meth. A 447 (2000) 1.

[142] A. Bardi et al., Nucl. Instrum. Meth. A 485 (2002) 178.

[143] C.S. Hill, Nucl. Instrum. Meth. A 530 (2004) 1.

[144] A. Abulencia et al., Phys. Rev. Lett. 97 (2006) 242003.

[145] A. Affolder et al., Nucl. Instr. and Meth. A 453 (2000) 84.

[146] T. Affolder et al., Nucl. Instr. and Meth. A 526 (2004) 249.

[147] D. Acosta et al., Nucl. Instr. and Meth. A 518 (2004) 605.

[148] P. de Barbaro, AIP Conf. Proc. 450 (1998) 405.

[149] V.M. Abazov et al., Nucl. Instrum. Meth. A 565 (2006) 463.

[150] W.E. Cooper, Nucl. Instrum. Meth. A598 (2009) 41.

[151] V.M. Abazov et al., Phys. Rev. D 74 (2006) 112004.

[152] L. Evans and P. Bryant, JINST 3 (2008) S08001.

[153] G. Aad et al., JINST 3 (2008) S08003.

[154] G. Lindstrom et al., Nucl. Instrum. Meth. A 466 (2001) 308.

[155] R. Adolphi et al., JINST 0803 (2008) S08004.

[156] R. Kalman, Transactions of the ASME-Journal of Basic Engineering, Series D 82 (1960) 35.

[157] V.M. Abazov et al., Phys. Rev. D 76 (2007) 092007.

[158] A. Abulencia et al., Phys. Rev. D 74 (2006) 072006.

[159] G.L. Bayatian et al., CMS physics: Technical design report, CERN-LHCC-2006-001.

[160] R. Fruhwirth and T. Speer, Nucl. Instrum. Meth. A 534 (2004) 217.

[161] H. Bethe and W. Heitler, Proc. Roy. Soc. Lond. A 146 (1934) 83.

[162] M.H. Seymour and C. Tevlin, JHEP 11 (2006) 052.

[163] T. Aaltonen et al., Phys. Rev. D 76 (2007) 072009.

[164] A. Abulencia et al., Phys. Rev. Lett. 97 (2006) 082004. 
[165] D. Buskulic et al., Phys. Lett. B 313 (1993) 535.

[166] F. Abe et al., Phys. Rev. D 50 (1994) 2966.

[167] D. Acosta et al., Phys. Rev. D 72 (2005) 032002.

[168] D. Acosta et al., Phys. Rev. D 71 (2005) 052003.

[169] V.M. Abazov et al., Phys. Lett. B 626 (2005) 35.

[170] V. Shary, Nuovo Cim. 123B (2008) 1053.

[171] M. Schmaltz and D. Tucker-Smith, Ann. Rev. Nucl. Part. Sci. 55 (2005) 229.

[172] C.T. Hill and S.J. Parke, Phys. Rev. D 49 (1994) 4454.

[173] H.P. Nilles, Phys. Rept. 110 (1984) 1.

[174] M.L. Mangano et al., JHEP 07 (2003) 001.

[175] F. Maltoni and T. Stelzer, JHEP 02 (2003) 027.

[176] T. Sjöstrand, Comp. Phys. Commun. 135 (2001) 238.

[177] G. Corcella et al., JHEP 01 (2001) 010.

[178] D.J. Lange, Nucl. Instrum. Meth. A 462 (2001) 152.

[179] S. Agostinelli et al., Nucl. Instrum. Meth. A 506 (2003) 250.

[180] J. Allison et al., IEEE Trans. Nucl. Sci. 53 (2006) 270.

[181] T. Aaltonen et al., CDF Conf. Note 9399, 2008.

[182] D. Acosta et al., Phys. Rev. Lett. 93 (2004) 142001.

[183] T. Aaltonen et al., Phys. Rev. D 79 (2009) 112007.

[184] V.M. Abazov et al., Phys. Rev. D 76 (2007) 052006.

[185] V.M. Abazov et al., arXiv: 0901.2137 [hep-ex] (2009).

[186] A. Abulencia et al., Phys. Rev. D 78 (2008) 012003.

[187] A. Abulencia et al., Phys. Rev. Lett. 96 (2006) 202002.

[188] T. Aaltonen et al., Phys. Rev. D 79 (2009) 052007.

[189] T. Aaltonen et al., CDF Conf. Note 9474, 2008.

[190] T. Aaltonen et al., CDF Conf. Note 9616, 2008.

[191] F. Abe et al., Phys. Rev. Lett. 79 (1997) 1992.

[192] B. Abbott et al., Phys. Rev. Lett. 83 (1999) 1908.

[193] V.M. Abazov et al., arXiv: 0903.5525 [hep-ex] (2009).

[194] L. Lyons, A.J. Martin and D.H. Saxon, Phys. Rev. D 41 (1990) 982.

[195] M.L. Mangano, Int. J. Mod. Phys. A 23 (2008) 3833.

[196] D. Acosta et al., Phys. Rev. D 65 (2002) 091102.

[197] D. Acosta et al., Phys. Rev. D 69 (2004) 052003.

[198] D. Acosta et al., Phys. Rev. D 71 (2005) 012005.

[199] B. Abbott et al., Phys. Rev. D 63 (2000) 031101.

[200] V.M. Abazov et al., Phys. Lett. B 517 (2001) 282.

[201] V.M. Abazov et al., Phys. Lett. B 622 (2005) 265.

[202] V.M. Abazov et al., Phys. Rev. D 75 (2007) 092007.

[203] V.M. Abazov et al., Phys. Rev. Lett. 98 (2007) 181802.

[204] V.M. Abazov et al., Phys. Rev. D 78 (2008) 012005.

[205] T. Aaltonen et al., Phys. Rev. Lett. 101 (2008) 252001. 
[206] T. Aaltonen et al., arXiv: 0903.0885 [hep-ex] (2009).

[207] V.M. Abazov et al., arXiv: 0903.0850 [hep-ex] (2009).

[208] M. Bobrowski et al., arXiv: 0902.4883 [hep-ph] (2009).

[209] J. Alwall et al., Eur. Phys. J. C 49 (2007) 791.

[210] A. Bhatti et al., Nucl. Instrum. Meth. A 566 (2006) 375.

[211] T. Scanlon, FERMILAB-THESIS-2006-43.

[212] T. Sjostrand, S. Mrenna and P. Skands, JHEP 05 (2006) 026.

[213] J. Alwall et al., JHEP 09 (2007) 028.

[214] J. Lueck, FERMILAB-MASTERS-2006-01.

[215] E.E. Boos et al., Phys. Atom. Nucl. 69 (2006) 1317.

[216] E. Boos et al., Nucl. Instrum. Meth. A 534 (2004) 250.

[217] T.M.P. Tait and C.P. Yuan, Phys. Rev. D 63 (2001) 014018.

[218] E.H. Simmons, arXiv: hep-ph/9908511 (1998).

[219] F. Abe et al., Phys. Rev. D 50 (1994) 2966.

[220] T. Aaltonen et al., CDF note 9679, 2009 (unpublished) .

[221] K. Kondo, J. Phys. Soc. Jpn. 57 (1988) 4126.

[222] K. Kondo, J. Phys. Soc. Jpn. 60 (1991) 836.

[223] K. Kondo, T. Chikamatsu and S.H. Kim, J. Phys. Soc. Jpn. 62 (1993) 1177.

[224] K. Kondo, arXiv: hep-ex/0508035 (2005).

[225] R.H. Dalitz and G.R. Goldstein, Phys. Rev. D 45 (1992) 1531.

[226] R.H. Dalitz and G.R. Goldstein, Phys. Lett. B 287 (1992) 225.

[227] R.H. Dalitz and G.R. Goldstein, Proc. R. Soc. Lond. A 445 (1999) 2803.

[228] V.M. Abazov et al., DØ note 5877, 2009 (unpublished) .

[229] V.M. Abazov et al., Phys. Rev. D 75 (2007) 092001.

[230] P. Abreu et al., Eur. Phys. J. C 2 (1998) 581.

[231] M. Mulders, Int. J. Mod. Phys. A 16S1A (2001) 284.

[232] F. Fiedler, Eur. Phys. J. C 53 (2008) 41.

[233] C.S. Hill, J.R. Incandela and J.M. Lamb, Phys. Rev. D 71 (2005) 054029.

[234] A. Abulencia et al., Phys. Rev. D 75 (2007) 071102.

[235] T. Aaltonen et al., CDF note 9414, 2008 (unpublished) .

[236] L. Sonnenschein, Phys. Rev. D 73 (2006) 054015.

[237] B. Abbott et al., Phys. Rev. Lett. 80 (1998) 2063.

[238] B. Abbott et al., Phys. Rev. D 60 (1999) 052001.

[239] F. Abe et al., Phys. Rev. Lett. 82 (1999) 271.

[240] A. Abulencia et al., Phys. Rev. Lett. 96 (2006) 152002.

[241] D. Acosta et al., CDF conf. note 7797, 2005 (unpublished) .

[242] V.M. Abazov et al., DØ note 5746, 2009 (unpublished) .

[243] V.M. Abazov et al., DØ note 5463, 2007 (unpublished) .

[244] T. Aaltonen et al., Phys. Rev. Lett. 102 (2009) 152001.

[245] D. Acosta et al., CDF conf. note 8959, 2007 (unpublished) .

[246] V.M. Abazov et al., DØ note 5459, 2009 (unpublished) . 
[247] T. Aaltonen et al., CDF note 9694, 2009 (unpublished) .

[248] B. Abbott et al., Phys. Rev. D 58 (1998) 052001.

[249] S. Abachi et al., Phys. Rev. Lett. 79 (1997) 1197.

[250] V.M. Abazov et al., Nature 429 (2004) 638.

[251] V.M. Abazov et al., arXiv: hep-ex/0407005 (2004).

[252] V.M. Abazov et al., Phys. Lett. B 606 (2005) 25.

[253] V.M. Abazov et al., DØ note 5907, 2009 (unpublished) .

[254] V.M. Abazov et al., Phys. Rev. Lett. 101 (2008) 182001.

[255] V.M. Abazov et al., DØ note 5897, 2009 (unpublished) .

[256] V.M. Abazov et al., arXiv: hep-ex/09012137 (2009).

[257] V.M. Abazov et al., DØ note 5900, 2009 (unpublished) .

[258] F. Abe et al., Phys. Rev. Lett. 80 (1998) 2767.

[259] T. Affolder et al., Phys. Rev. D 63 (2001) 032003.

[260] F. Abe et al., Phys. Rev. Lett. 79 (1997) 1992.

[261] A. Abulencia et al., CDF note 9518, 2009 (unpublished) .

[262] A. Abulencia et al., Phys. Rev. Lett. 99 (2007) 182002, hep-ex/0703045.

[263] T. Aaltonen et al., CDF note 9692, 2009 (unpublished) .

[264] A. Abulencia et al., CDF note 8669, 2007 (unpublished) .

[265] T. Aaltonen et al., CDF note 9683, 2009 (unpublished) .

[266] A. Abulencia et al., CDF note 9135, 2007 (unpublished) .

[267] A. Abulencia et al., CDF note 8951, 2009 (unpublished) .

[268] A. Abulencia et al., Phys. Rev. D 75 (2007) 031105.

[269] A. Abulencia et al., CDF note 8955, 2007 (unpublished) .

[270] T. Aaltonen et al., arXiv: 0901.3773 [hep-ex].

[271] T. Aaltonen et al., Phys. Rev. Lett. 100 (2008) 062005.

[272] T. Aaltonen et al., CDF note 9265, 2008 (unpublished) .

[273] T. Aaltonen et al., arXiv: 0811.1062 [hep-ex].

[274] A. Abulencia et al., CDF note 9714, 2009 (unpublished) .

[275] P. Azzi et al., arXiv: hep-ex/0404010 (2004).

[276] A.H. Hoang and I.W. Stewart, Nucl. Phys. Proc. Suppl. 185 (2008) 220.

[277] S. Kuhlman et al., FERMILAB-PUB-96-112 (unpublished), arXiv: hep-ex/9605011 (1996).

[278] A. Abulencia et al., Phys. Rev. D 75 (2007) 052001.

[279] T. Aaltonen et al., Phys. Lett. B 674 (2009) 160.

[280] V.M. Abazov et al., Phys. Rev. D 75 (2007) 031102.

[281] V.M. Abazov et al., Phys. Rev. Lett. 100 (2008) 062004.

[282] A. Abulencia et al., Phys. Rev. Lett. 98 (2007) 072001.

[283] L. Lyons, D. Gibaut and P. Clifford, Nucl. Instrum. Meth. A 270 (1988) 110.

[284] T. Aaltonen et al., CDF conf. note 9144 (2007).

[285] C. Amsler et al., Phys. Lett. B 667 (2008) 1.

[286] D. Acosta et al., Phys. Rev. Lett. 95 (2005) 102002.

[287] V.M. Abazov et al., Phys. Rev. Lett. 100 (2008) 192003. 
[288] H. Fritzsch, Phys. Lett. B 224 (1989) 423.

[289] J.A. Aguilar-Saavedra, Acta Phys. Polon. B 35 (2004) 2695.

[290] T. Aaltonen et al., Phys. Rev. Lett. 102 (2009) 151801.

[291] V.M. Abazov et al., Phys. Rev. Lett. 99 (2007) 191802.

[292] F. Abe et al., Phys. Rev. Lett. 80 (1998) 2525.

[293] T. Aaltonen et al., Phys. Rev. Lett. 101 (2008) 192002.

[294] A. Heister et al., Phys. Lett. B 543 (2002) 173.

[295] J. Abdallah et al., Phys. Lett. B 590 (2004) 21.

[296] P. Achard et al., Phys. Lett. B 549 (2002) 290.

[297] G. Abbiendi et al., Phys. Lett. B 521 (2001) 181.

[298] S. Chekanov et al., Phys. Lett. B 559 (2003) 153.

[299] A. Aktas et al., Eur. Phys. J. C 33 (2004) 9.

[300] H1, Search for single top quark production in e p collisions at HERA, Contributed paper to EPS2007, abstract 776, H1prelim-07-163, 2007.

[301] J.J. Liu et al., Phys. Rev. D 72 (2005) 074018.

[302] L.L. Yang et al., Phys. Rev. D 73 (2006) 074017.

[303] J.J. Zhang et al., Phys. Rev. Lett. 102 (2009) 072001.

[304] T. Aaltonen et al., Phys. Rev. Lett. 101 (2008) 202001.

[305] T. Aaltonen et al., CDF conf. note 9724 (2009).

[306] V.M. Abazov et al., Phys. Rev. Lett. 100 (2008) 142002.

[307] O. Antunano, J.H. Kuhn and G. Rodrigo, Phys. Rev. D 77 (2008) 014003.

[308] S. Dittmaier, P. Uwer and S. Weinzierl, Phys. Rev. Lett. 98 (2007) 262002.

[309] T. Aaltonen et al., Phys. Rev. Lett. 102 (2009) 222003.

[310] A. Hocker and V. Kartvelishvili, Nucl. Instrum. Meth. A 372 (1996) 469.

[311] N. Kidonakis and R. Vogt, Phys. Rev. D 68 (2003) 114014.

[312] T. Arens and L.M. Sehgal, Phys. Lett. B 302 (1993) 501.

[313] T. Aaltonen et al., CDF conf. note 9432 (2008).

[314] T. Aaltonen et al., Phys. Rev. D 79 (2009) 031101.

[315] T. Aaltonen et al., Phys. Rev. Lett. 102 (2009) 042001.

[316] T. Aaltonen et al., CDF conf. note 8104 (2006).

[317] A. Stange and S. Willenbrock, Phys. Rev. D 48 (1993) 2054.

[318] T.F. Feng, X.Q. Li and J. Maalampi, Phys. Rev. D 69 (2004) 115007.

[319] J.A. Aguilar-Saavedra, JHEP 12 (2006) 033.

[320] V.M. Abazov et al., D $\varnothing$ conf. note 5739 (2008).

[321] A. Leike, Phys. Rept. 317 (1999) 143.

[322] B. Lillie, L. Randall and L.T. Wang, JHEP 09 (2007) 074.

[323] T.G. Rizzo, Phys. Rev. D 61 (2000) 055005.

[324] L.M. Sehgal and M. Wanninger, Phys. Lett. B 200 (1988) 211.

[325] R.M. Harris, C.T. Hill and S.J. Parke, arXiv: hep-ph/9911288 (1999).

[326] T. Aaltonen et al., Phys. Rev. D 77 (2008) 051102.

[327] T. Aaltonen et al., Phys. Rev. Lett. 100 (2008) 231801. 
[328] T. Aaltonen et al., CDF conf. note 9164 (2008).

[329] A. Abulencia et al., Phys. Rev. D 73 (2006) 092002.

[330] V.M. Abazov et al., Phys. Lett. B 668 (2008) 98.

[331] V.M. Abazov et al., DØ conf. note 5600 (2008).

[332] V.M. Abazov et al., DØ conf. note 5882 (2009).

[333] V.M. Abazov et al., Phys. Rev. Lett. 102 (2009) 191802.

[334] E. Boos et al., Phys. Lett. B 655 (2007) 245.

[335] T. Aaltonen et al., arXiv: 0902.3276 [hep-ex] (2009).

[336] V.M. Abazov et al., Phys. Rev. Lett. 100 (2008) 211803.

[337] Z. Sullivan, Phys. Rev. D 66 (2002) 075011.

[338] A. Abulencia et al., Phys. Rev. Lett. 96 (2006) 042003.

[339] T. Aaltonen et al., CDF conf. note 9322 (2008).

[340] V.M. Abazov et al., Phys. Lett. B 674 (2009) 4.

[341] G.D. Kribs et al., Phys. Rev. D 76 (2007) 075016.

[342] T. Han et al., Phys. Lett. B 563 (2003) 191.

[343] T. Aaltonen et al., Phys. Rev. Lett. 100 (2008) 161803.

[344] T. Aaltonen et al., CDF conf. note 9446 (2008).

[345] R. Bonciani et al., Nucl. Phys. B 529 (1998) 424. 